\title{
Theoretical Investigation of Variable Area Ejectors
}

\author{
by
}

\section{Mikhail Koupriyanov}

\author{
A Thesis submitted to \\ the Faculty of Graduate Studies and Research \\ in partial fulfillment of \\ the requirements for the degree of \\ Master of Applied Science
}

\author{
Ottawa-Carleton Institute for \\ Mechanical and Aerospace Engineering \\ Department of Mechanical and Aerospace Engineering \\ Carleton University \\ Ottawa, Ontario, Canada
}

September 2008

Copyright (C)

2008 - Mikhail Koupriyanov 


$\begin{array}{ll}\begin{array}{l}\text { Library and } \\ \text { Archives Canada }\end{array} & \begin{array}{l}\text { Bibliothèque et } \\ \text { Archives Canada }\end{array} \\ \begin{array}{l}\text { Published Heritage } \\ \text { Branch }\end{array} & \begin{array}{l}\text { Direction du } \\ \text { Patrimoine de l'édition }\end{array} \\ \begin{array}{l}\text { 395 Wellington Street } \\ \text { Ottawa ON K1A 0N4 } \\ \text { Canada }\end{array} & \begin{array}{l}\text { 395, rue Wellington } \\ \text { Ottawa ON K1A 0N4 } \\ \text { Canada }\end{array}\end{array}$

Your file Votre référence ISBN: 978-0-494-44048-3 Our file Notre référence ISBN: 978-0-494-44048-3

NOTICE:

The author has granted a nonexclusive license allowing Library and Archives Canada to reproduce, publish, archive, preserve, conserve, communicate to the public by telecommunication or on the Internet, loan, distribute and sell theses worldwide, for commercial or noncommercial purposes, in microform, paper, electronic and/or any other formats.

The author retains copyright ownership and moral rights in this thesis. Neither the thesis nor substantial extracts from it may be printed or otherwise reproduced without the author's permission.
AVIS:

L'auteur a accordé une licence non exclusive permettant à la Bibliothèque et Archives Canada de reproduire, publier, archiver, sauvegarder, conserver, transmettre au public par télécommunication ou par l'Internet, prêter, distribuer et vendre des thèses partout dans le monde, à des fins commerciales ou autres, sur support microforme, papier, électronique et/ou autres formats.

L'auteur conserve la propriété du droit d'auteur et des droits moraux qui protège cette thèse. $\mathrm{Ni}$ la thèse ni des extraits substantiels de celle-ci ne doivent être imprimés ou autrement reproduits sans son autorisation.
In compliance with the Canadian Privacy Act some supporting forms may have been removed from this thesis.

While these forms may be included in the document page count, their removal does not represent any loss of content from the thesis.
Conformément à la loi canadienne sur la protection de la vie privée, quelques formulaires secondaires ont été enlevés de cette thèse.

Bien que ces formulaires aient inclus dans la pagination, il n'y aura aucun contenu manquant.

\section{Canada}




\section{Abstract}

A theoretical analysis of a variable area ejector is presented. The flowfield is solved using a steady quasi-one-dimensional, inviscid control volume formulation for cases of both complete and incomplete mixing while combustion effects are included with an equilibrium calculation. An assumed three parameter analytical wall pressure distribution is used in all cases. Under fully mixed-conditions, the model estimated compression ratios that were $40 \%$ higher than the computational values due to neglecting turbulence and viscosity. This cause was later confirmed with a partially mixed calculation which also predicted a mixing length of 9 diameters. Under SMC conditions, improved compression was achieved at an equivalence ratio of 2.5 , while a decrease in performance occurred at Stoichiometric conditions. The oxidation of carbon monoxide occurred for the entire equivalence ratio range and was responsible for the majority of the heat release in the ejector. Thrust augmentation was found to increase with area constriction up to a limit, with the Stoichiometric case yielding values as high as $12 \%$. 


\section{Acknowledgements}

My time at Carleton University has been a rewarding and exciting experience which I will always remember. I would like to extend my thanks to the department faculty and staff for making me feel welcome in this university which seemed foreign not so long ago.

I also want to take this opportunity to thank my adviser, Dr. Jason Etele for giving me the opportunity to work on a challenging and interesting project, which has greatly extended my understanding of high speed aerodynamics and combustion (my favorite engineering disciplines). Your guidance and desire to push me to do better has made me a far more diligent researcher and has greatly improved the quality of the work which I am now submitting.

Last, but not least, I would like to a acknowledge the Natural Sciences and Engineering Research Council, who's financial assistance made it possible for me to move to Ottawa and pursue my Masters degree full time. 


\section{Table of Contents}

Nomenclature $\quad$ ix

1 Introduction 1

1.1 Background ........................ 1

1.2 Literature Review . . . . . . . . . . . . . . . . . . . . 4

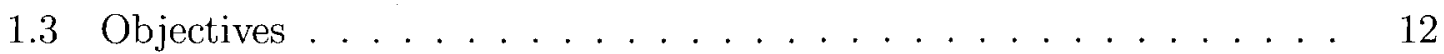

2 Non-Reacting Ejector Theory $\quad 14$

2.1 Wall Pressure Distribution . . . . . . . . . . . . . . . . . . . 19

2.2 Solution for Minimum Rocket Pressure . . . . . . . . . . . . . . . 20

2.2.1 Control Volume Solution . . . . . . . . . . . . 21

2.2 .2 Riemann Solution . . . . . . . . . . . . . . . 24

2.3 Wall Pressure Integral f . . . . . . . . . . . . . . . . . . . . . . . 28

2.4 Numerical Solution . . . . . . . . . . . . . . . . . . 30

2.4.1 CV Method - Numerical Solution . . . . . . . . . . . . 34

2.4 .2 Area-Mach Number Relation . . . . . . . . . . . . . 38

3 Theory for SMC Ejector $\quad 40$

3.1 Gibbs Minimization . . . . . . . . . . . . . . . . . . . 42

3.2 Numerical Solution . . . . . . . . . . . . . . . . . 44 
4 Partially Mixed Ejector Theory $\quad 51$

4.1 Mixed-Flow Velocity Profile . . . . . . . . . . . . . . . 54

4.2 Mixing Correlations . . . . . . . . . . . . . . . 57

4.3 Numerical Solution . . . . . . . . . . . . . . . . . . . 59

5 Results \& Discussion $\quad 61$

5.1 Pressure Distribution . . . . . . . . . . . . . . . . . . . . 61

5.2 Ejector Performance . . . . . . . . . . . . . 65

5.3 Effects of Combustion . . . . . . . . . . . . . . . 69

5.4 Effects of Mixing . . . . . . . . . . . . . . . . 83

5.5 Thrust Augmentation . . . . . . . . . . . . . . . . . . . 89

6 Conclusions $\quad 94$

6.1 Future Work . . . . . . . . . . . . . . . . . . . 97 


\section{List of Figures}

1.1 Typical RBCC Engine $\ldots \ldots \ldots \ldots \ldots \ldots \ldots$

1.2 Specific Impulse of Various Propulsive Cycles $[17] \ldots \ldots \ldots$

2.1 Ejector Control Volume $\quad \therefore \ldots \ldots \ldots \ldots$

2.2 Air \& Rocket Control Volumes for Estimating $p_{\min } \ldots \ldots \ldots \ldots$

2.3 Riemann Problem Set-Up Adapted to Ejector . . . . . . . . . . . 24

2.4 Velocity Polygon at Node $i \ldots \ldots \ldots \ldots$

2.5 Ejector Control Volume showing surface element $\mathrm{d} \vec{S} \ldots \ldots \ldots$

2.6 Ejector Solution Process (Non-Reacting) $\ldots \ldots \ldots \ldots$

2.7 CV-based Solution Process for $p_{\min } \ldots \ldots \ldots \ldots \ldots$

2.8 Solution of Area-Mach Number Relation . . . . . . . . . . . . 39

3.1 Ejector Solution Process (Reacting) . . . . . . . . . . . . . 50

4.1 Assumed Velocity Profile at Mixed-Flow Plane . . . . . . . . . . 55

4.2 Velocity Profile at Ejector Inlet . . . . . . . . . . . . . 58

$5.1 \quad$ Wall Pressure Distributions $\ldots \ldots \ldots \ldots$

5.2 Minimum Rocket Stream Pressure at a Fixed Air Mass Flow Rate . . 63

5.3 Ejector Compression Ratio $\pi_{m}$, theoretical (dashed lines) and CFD (solid line) results . . . . . . . . . . . . . . . 66 
5.4 Ejector Compression Augmentation $\bar{\pi}_{m}$, theoretical (dashed lines) and CFD (solid line) results . . . . . . . . . . . . . . . . 68

5.5 Ejector Compression Under SMC Conditions . . . . . . . . . . . 71

5.6 Relative Change in Combustion Heat Release $\left(C_{R}=0.15\right) \quad \ldots \ldots 72$

5.7 Compression Augmentation at SMC Operation $\left(C_{R}=0.15\right) \quad \ldots \quad 73$

5.8 Relative Changes in Pressure and Mach Number $\left(C_{R}=0.15\right) \ldots \ldots 74$

5.9 Ejector Mixed-Flow Temperature $\left(C_{R}=0.15\right) \ldots \ldots \ldots \ldots$

5.10 Effect of Rocket Temperature on Ejector Compression $\left(C_{R}=0.15\right)$. 76

5.11 Ejector Composition - Major Species $\left(C_{R}=0.15\right) \ldots \ldots \ldots 78$

5.12 Ejector Composition - Minor Species $\left(C_{R}=0.15\right) \ldots \ldots \ldots$

5.13 Production of $\mathrm{CO}_{2} \& \mathrm{H}_{2} \mathrm{O}\left(\mathrm{C}_{R}=0.15\right) \ldots \ldots \ldots \ldots$

5.14 Momentum Mixing Parameter . . . . . . . . . . . . . . 84

5.15 Energy Mixing Parameter . . . . . . . . . . . 85

5.16 Effect of Ejector Duct Length _ . . . . . . . . . . . 86

5.17 Velocity Profiles at Mixed-Flow Plane for Various $L / D$ 's . . . . . 87

5.18 Velocity $u^{*}$ from Eq. $(4.17) \ldots \ldots \ldots \ldots \ldots$

5.19 Calculation of Ejector Thrust . . . . . . . . . . . 90

5.20 Ejector Performance Map _. . . . . . . . . . . . . . . 91

5.21 Thrust Augmentation and Air Entrainment $\left(C_{R}=0.3\right) \ldots \ldots . .92$ 


\section{List of Tables}

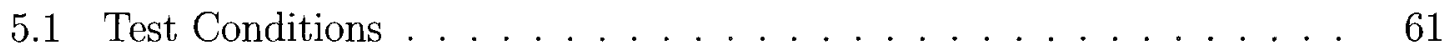

5.2 Comparison of Flow Variables at Point of Maximum Expansion for

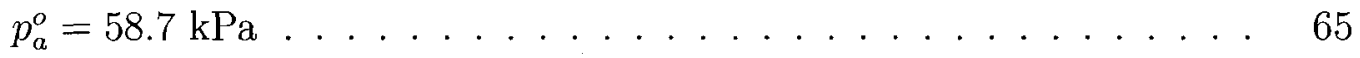

5.3 Assumed Product Species _ . . . . . . . . . . . . 70

5.4 Ejector Configuration . . . . . . . . . . . . . 90 


\section{Nomenclature}

\section{Roman Symbols}

A Area

a speed of sound

$b_{i} \quad$ total flow rate

of atomic species $i$

$c_{i} \quad$ coefficient

$C_{p} \quad$ specific heat at constant

pressure

$C_{R} \quad$ contraction ratio $1-A_{m} / A_{i}$

D diameter

$f \quad$ Newton-Raphson functional

$\bar{g} \quad$ gibbs free energy (molar)

$\bar{g}_{f}^{o} \quad$ gibbs free energy of

formation (molar)

$\Delta H_{R}$ heat of reaction

$\bar{h} \quad$ molar enthalpy

$h \quad$ specific enthalpy $\bar{h}_{f}^{o} \quad$ standard heat of formation (molar)

$L \quad$ length of ejector duct

$\dot{m} \quad$ mass flow rate

$M \quad$ Mach number

$M_{c} \quad$ convective Mach number

$\hat{n} \quad$ unit normal vector

$N \quad$ molar flow rate

$n_{a} \quad$ number of distinct

atomic species

$n_{r} \quad$ number of species in

rocket exhaust

$n_{s} \quad$ number of species

$p \quad$ pressure

$R \quad$ gas constant

$r \quad$ radius

$R_{u} \quad$ universal gas constant 
$s \quad$ turbulent spreading

parameter

$s_{\theta} \quad$ turbulent spreading

parameter based on

momentum thickness

$T \quad$ temperature

$t \quad$ time

$u \quad$ streamwise velocity

$u_{1} \quad$ velocity at upper boundary

of shear layer

$u_{2} \quad$ velocity at lower boundary

of shear layer

$u^{*} \quad$ dimensionless velocity

$u_{2} / u_{1}$

$\vec{V} \quad$ resultant velocity

$v \quad$ radial velocity

$V_{c s} \quad$ contact surface velocity

W molecular weight

$x$ axial coordinate

$Y \quad$ mass fraction

\section{Greek Symbols}

$\alpha \quad$ air/rocket mass flow ratio

$\beta_{e} \quad$ energy mixing factor

$\beta_{m} \quad$ momentum mixing factor

$\gamma \quad$ ratio of specific heats

$\delta \quad$ shear layer thickness,

flow angle

$\epsilon \quad$ relative shear layer thickness

$\delta_{m} / r_{m}$

$\zeta \quad$ rocket/air total pressure

ratio, $p_{r}^{o} / p_{a}^{o}$

$\eta_{i k} \quad$ amount of atomic particle $i$ per kmol of species $k$

$\theta \quad$ air/rocket specific total

enthalpy ratio

$\theta_{o} \quad$ initial momentum thickness

$\lambda \quad$ Lagrange multiplier

$\mu \quad$ functional

$\nu \quad$ density ratio, $\rho_{r} / \rho_{a}$

$\xi \quad$ non-dimensional shear layer coordinate 


\begin{tabular}{|c|c|c|c|}
\hline$\pi_{m}$ & compression ratio, $p_{m}^{o} / p_{a}^{o}$ & $i$ & ejector inlet \\
\hline \multirow[t]{2}{*}{$\bar{\pi}_{m}$} & compression augmentation & $m$ & mixed flow (ejector exit) \\
\hline & ratio & $r$ & rocket \\
\hline$\rho$ & density & ref & reference conditions \\
\hline$\sigma$ & rocket exhaust/ejector & & $(273 \mathrm{~K}, 1 \mathrm{~atm})$ \\
\hline & inlet area ratio & $w$ & wall \\
\hline$\tau$ & velocity ratio, $u_{r} / u_{a}$ & \multicolumn{2}{|c|}{ Superscripts } \\
\hline$\Upsilon$ & $\begin{array}{l}\text { numerical damping } \\
\text { parameter }\end{array}$ & 0 & stagnation conditions \\
\hline$\Phi$ & thrust augmentation ratio & $*$ & sonic conditions \\
\hline & $F_{e j} / F_{r}$ & - & area-averaged quantity \\
\hline$\phi_{r}$ & $\begin{array}{l}\text { equivalence ratio of the } \\
\text { rocket's combustion chamber }\end{array}$ & $\sim$ & normalized by $R_{u} T_{m}$ \\
\hline
\end{tabular}

\section{Subscripts}

2 point of maximum expansion

a air
$A M \quad$ Area-Mach Number relation
$C V$ control volume

$D S L$ dividing streamline

e propelling nozzle exit 


\section{Chapter 1}

\section{Introduction}

\subsection{Background}

Currently the only available means of orbital insertion is by using chemical rocket engines. Although rocket propulsion offers a high thrust-to-weight ratio, it suffers from a relatively low specific impulse (on the order of 300s), as well as the burden of having to carry a large quantity of on-board oxidizer. These shortcomings collectively make launching payloads into space a very expensive endeavour with costs on the order of $\$ 22000 / \mathrm{kg}$ of payload. The desire to bring down these costs served as the impetus behind the Highly Reusable Space Transportation Study released by NASA in 1997. The goal of the study was to identify technologies which would reduce the costs of space access by a significant amount. Among the candidates which showed promise of achieving the cost reduction goal were various types of air-breathing CombinedCycle Propulsion (CCP) systems. A combined cycle engine essentially integrates different propulsive cycles into a single cngine/flowpath architecture. One of the variants of such a system is the Rocket-Based Combined-Cycle Engine (RBCC) which has at its core a chemical rocket, required for space flight and static thrust. An example of a generic RBCC engine is shown in Figure 1.1. 


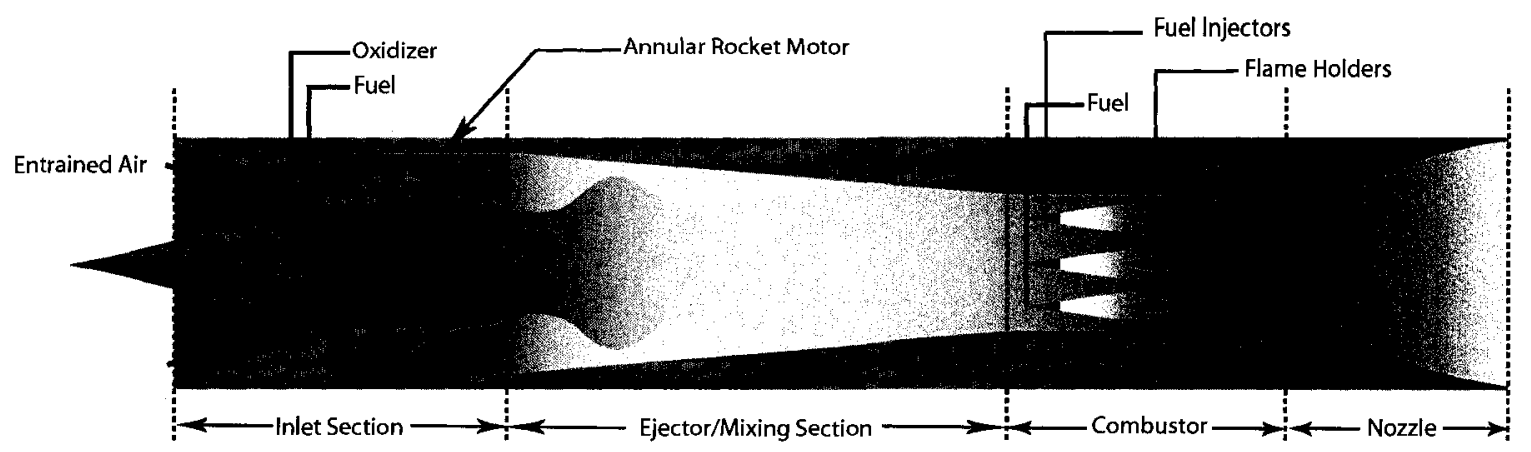

Figure 1.1: Typical RBCC Engine

An RBCC engine typically operates in four flight modes, namely the rocket ejector mode, the ramjet mode, the scramjet mode and finally a pure rocket mode. The low speed rocket ejector mode operates from static conditions up into the low supersonic Mach number range $(M=3)$. In this mode, the pumping action of the rocket (primary) stream entrains and compresses atmospheric air through a turbulent mixing process. The increased total pressure of the entrained air coupled with a higher mass flow rate through the engine acts to increase the thrust and specific impulse of the engine at these speeds. Further thrust augmentation in the ejector mode is achieved by combusting additional fuel. Around $M=3$ the RBCC engine transitions into ramjet mode. In this case, additional compression is achieved through the inlet shock structure, while the combustion of the incoming atmospheric air occurs subsonically. When the flight Mach number becomes too high to sustain subsonic combustion, the engine transitions into scramjet mode (around $M=6$ ), in which case the rocket is usually shut off. Finally, when the altitude is high enough, at which point the flight Mach number is around $M=12-15$, the air inlet of the RBCC engine is usually closed and the engine transitions to pure rocket operation. This mode is then used for the remainder of the vehicles ascent into orbit.

There are several reasons why an RBCC engine outperforms rockets. First, the amount of on-board oxidizer is significantly reduced due to the entrainment of atmo- 


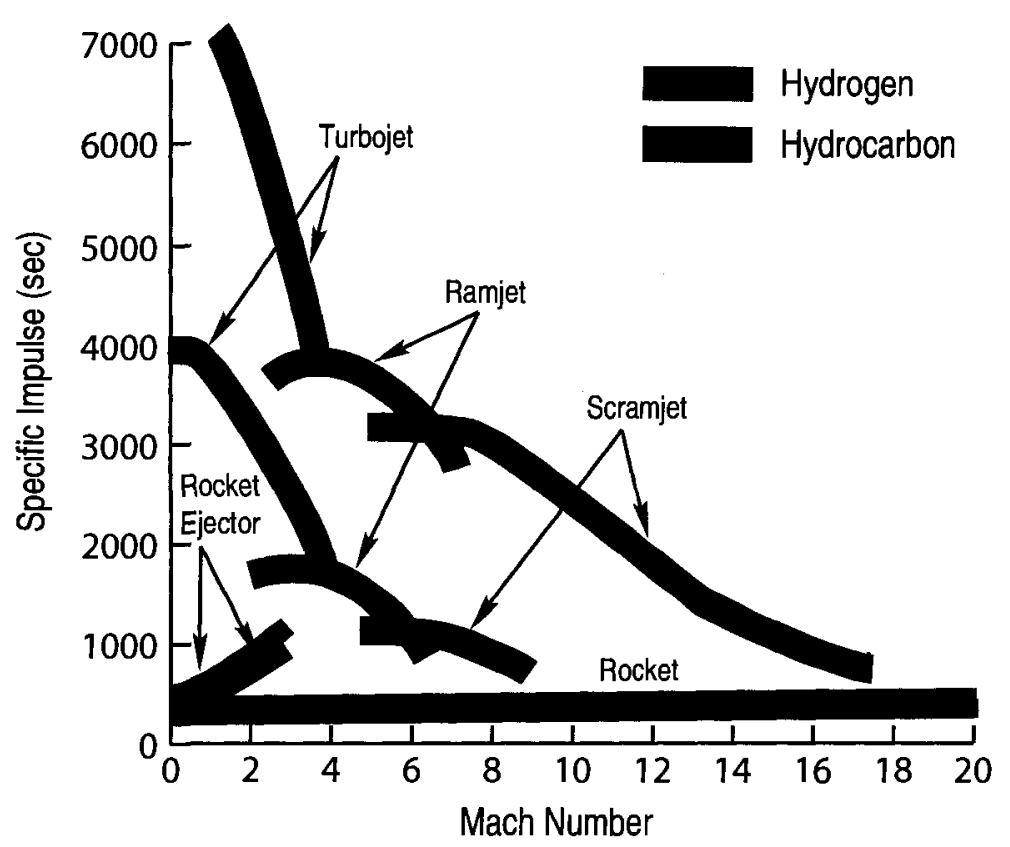

Figure 1.2: Specific Impulse of Various Propulsive Cycles [17]

spheric air, which could potentially decrease the vehicle's propellant mass fraction by $20 \%$ [17]. In their respective operating regimes, each of the engine's modes has a much higher specific impulse than the rocket (see Figure 1.2) and thus the specific impulse of the RBCC engine over the entire trajectory is increased substantially. Further benefits are attained from the high level of integration between the engine's operating cycles. Examples of these include using the rocket to entrain air in the ejector mode as well as a fuel injector in the ramjet mode. Furthermore, the RBCC engine duct can be used as a high expansion nozzle during rocket only operation [17]. All of these benefits make the RBCC engine an attractive alternative to rocket propulsion, as well as the focus of a considerable amount of research and development work. 


\subsection{Literature Review}

To date, this research has spawned numerous RBCC engine concepts all at different stages of development. Most of these emerged from an industry-based NASA funded study in the mid 1960's, which evaluated 36 potential engine designs to replace the multistage rocket propulsion system used in the space shuttle [26]. Twelve concepts, including the RBCC engine were identified as the most promising and were thus funded for further research [17]. The most promising ones were variants of an Ejector Scramjet (ESJ) with different subsystems installed to further improve performance. One of the configurations is Marquardt's Supercharged Ejector Scramjet (SESJ) [25]. The SESJ was designed to be used for future high speed space transportation systems and has been studied by Marquardt both conceptually and experimentally. The engine uses a dual concentric annular nozzle embedded inside an axisymmetric flowpath. The engine also houses an integrated fan stage which boosts the performance of the low-speed airbreathing modes (hence the term "supercharged"). Another relative of the ESJ is Aerojet's Strutjet engine [11]. This particular engine has a threedimensional asymmetric flowpath with embedded streamwise struts. The struts not only enhance mixing and compression within the engine but are also used to efficiently house various engine components such as the primary rockets and fuel injectors.

At low speeds $(M<1)$, the performance of the RBCC engine depends on the operation of the rocket ejector, which tends to produce the lowest levels of thrust augmentation as compared to the high speed modes. At these speeds, the performance of the ejector depends on three fundamental processes, namely the entrainment of atmospheric air, mixing of the two streams and finally the combustion of the rocket/air mixture within the engine duct. The entrainment of atmospheric air, for example, is required not only to increase the mass flow rate through the engine, but also to supply the fresh oxygen required for combustion. The entrained air must 
then fully mix with the rocket exhaust to achieve high compression as well as a uniform fuel/oxidizer distribution. Since longer ducts may be required to achieve a fully mixed flow, poor mixing can increase engine weight, reduce ejector compression and combustion efficiency thereby offsetting any performance gains.

The mixing kinetics inside an ejector can be studied in isolation by implementing so called cold flow experiments, where the primary rocket stream is replaced with either air or nitrogen at room temperature. This approach has the advantage of decoupling the mixing from the combustion, in an experimental sense, but lacks the realistic temperatures that are generally encountered in real ejectors. Despite this, there have been many studies, with the earliest dating back to the mid 1950's with the experimental/theoretical work of Fabri \& Paulon [30]. The purpose of their experiments was to characterize the operation of an ejector over a wide range of operating conditions. They were also among the first to identify the various operating regimes of the ejector, such as the supersonic regime (sometimes called a "Fabri Choke" [67]). In this regime, the high-pressure primary stream interacts with the entrained air flow and creates an aerodynamic throat which can choke the air stream.

More recent experimental studies have been aimed at reducing ejector mixing lengths through various means. For example, Kitamura et al. $[44,45]$ focused on the geometry of the ejector, and studied the performance of various mixing tube crosssections. In their work, pressure recovery lengths were used to quantify the mixing performance of axisymmetric, as well as straight and diverging rectangular geometries. The studies showed that the pressure recovery lengths of axisymmetric ducts were $50 \%$ shorter than the rectangular ones, and that the divergent mixing tubes suffered higher total pressure losses. Lineberry \& Landrum [49], went a different route and used a Strutjet-type experimental set-up to assess the use of multiple rocket nozzles in an ejector. Their results showed that higher rocket chamber pressures tended to increase the ejector mixing length, which was strongly dependent on the mass flow 
rate of the primary stream.

To study ejectors under more realistic operating conditions, cold flow experiments can be modified by heating the primary stream. This is done to experimentally quantify the effects that high temperatures have on ejector performance. Although basic thermodynamics can show that high temperatures increase impact losses in fully mixed ejectors, its effects on the turbulent mixing process require detailed experimental studies. One such study was carried out by Quinn [61], who demonstrated that the effect of high temperatures varied depending on the extent of mixing in the ejector. His data showed that a hot primary stream has a slightly favourable effect in short, partially mixed ejectors $(L / D \leq 6)$ due to the higher viscosity. In longer ejectors, on the other hand, where the flows are fully mixed, higher temperatures were shown to decrease air entrainment.

There have also been attempts to improve the mixing in the ejector by modifying the primary flow nozzle. Several concepts have been suggested, such as the use of so-called hypermixing nozzles [8] or forced mixer lobes [59]. Both methods derive from the concept of inducing large scale axial vorticity within the ejector to aid in mixing. This type of "convective" mixing is more efficient than shear mixing according to Presz et. al. [59], and can result in nearly complete mixing. The use of such mixing augmentation was originally intended to increase the thrust of ejectors used on VSTOL aircraft, while forced mixer lobes have been used in gas turbine engines. Subsonic studies were carried out by various researchers $[8,31,60]$ to evaluate different hypermixing nozzle arrangements. It was shown that the nozzles significantly enhanced mixing of the two streams, and high levels of thrust augmentation $(\approx 2)$ can be achieved [60]. It was later shown by Tillman et al. [66] that the same types of mixer lobes used by Presz et al. [59] in their subsonic experiments, work equally well for supersonic primary streams. In fact, his results showed that improved pumping and near ideal mixing can be achieved without flow separation. 
To boost the thrust of ejectors, extra fuel is generally injected into the engine to take advantage of the fresh oxygen that is being entrained. The extra fuel can be added by either running the rocket fuel rich and operating the ejector in the simultaneous mixing and combustion (SMC) scheme, or by allowing both streams to mix first and injecting extra fuel further downstream and thereby operating in diffusion and afterburning (DAB) mode. Thermodynamically, DAB tends to tends to have a higher specific impulse than SMC since the heat addition occurs at a much higher pressure [16]. The SMC mode, on the other hand is attractive because it requires a shorter duct and thus a potentially lighter engine. The choice of which mode to use in an ejector is clearly not an easy one, since either mode has its own advantages and the performance of each is strongly dependent on how its implemented.

The performance of a choked ejector running under SMC conditions was investigated by Masuya et al. [51]. In their work, wall pressure measurements combined with a one-dimensional theoretical analysis were used to deduce the axial distribution of flow properties. Their results showed that better mixing was achieved for high $\mathrm{O} / \mathrm{F}$ ratios as well as for longer combustors. Other studies in the area have been carried out with an emphasis on better understanding the parameters which have the greatest influcnce on the performance of the SMC mode [41,48]. For example, Li \& Liu [48] showed that the combustor geometry and the rocket chamber pressure $\left(p_{r}^{o}\right)$ both affect ejector thrust augmentation, with geometric effects being the most important. The authors also noted that the overall ejector equivalence ratio (taking into account all of the $\mathrm{O}_{2}$ in the ejector) should not exceed unity, otherwise the thrust augmentation would actually decrease.

Many ejector related studies, including those studying the effects of combustion (both DAB \& SMC) have come from Pennsylvania State University (PSU). In the work of Lehman et al. [46,47], Raman Spectroscopy, along with other measurement techniques were used to investigate the mixing characteristics of an ejector in both the 
SMC and DAB modes. Their measurements showed that in the SMC mode, mixing was finished earlier in the fuel rich case as compared to stoichiometric [46] and that the DAB mode tended to mix faster [47]. The same test apparatus was later used by Cramer et al. [14] to examine the effect of rocket thruster configuration in SMC mode. The dual thruster case was found to outperform the single thruster arrangement and was shown to entrain more air, mix faster and produce a higher pressure rise.

Given the increasing computational power of modern computers, many CFD-based ejector studies have been published to date. Work in this area generally involves the solution of the averaged (Reynolds or Favre), 2-D, Multi-Species Navier-Stokes Equations coupled with eddy viscosity-based turbulence models. Although combustion effects are usually considered, several studies investigated the effects of mixing without the inclusion of combustion kinetics. One of these is the work of Etele [26] which explored the effects of rocket placement and mixing duct area constriction on the performance of an ejector. His simulations showed that rockets placed around the annulus of the mixing duct promote the fastest mixing and that area constriction can improve ejector compression up to 30\% [27]. In contrast, Daines \& Bulman [15] used the unsteady oscillation of the primary jet flow direction to promote better mixing. Their results showed that using square wave switching increased the time-averaged thrust by $24 \%$ due mainly to the cyclic expulsion of high pressure, high velocity pockets of gas.

Several CFD-based studies including the effects of combustion have also been carried out. Simulations of this nature generally employ fairly simple combustion models (9 species finite rate $\mathrm{O}_{2} / \mathrm{H}_{2}$ models are common) to reduce computational costs. Daines \& Russel [16], for instance, used a single step global $\mathrm{H}_{2}-\mathrm{O}_{2}$ mechanism to qualitatively evaluate the performance of an ejector in both SMC and DAB. They found that the performance in SMC was generally poor and caused by combustion occurring too early in the mixing duct which tended to decrease the mixing rate. To 
improve performance, modifications to the SMC mode have been suggested, such as Shielded Primary Injection (SPI) [63]. In this method, fuel is injected into the primary stream in a manner that allows the rocket exhaust to shield it from the incoming air until significant mixing has occurred. Computational results have shown that SPI can outperform SMC by as much as 50\% (based on mixing duct pressures) [63] while maintaining a relatively short mixing duct (similar to SMC).

Due to the inherent complexity of such flowfields, most CFD codes used require extensive validation [26] and are rarely presented without supporting experimental data [50]. The reasons have to do with the predictive capability of modern CFD software, which is still not at a point where the results they produce can be fully trusted on their own. Despite the inherent uncertainties, CFD tools are still widely used, and have in fact been utilized to help explain experimental findings [69] by supplementing the available data. In addition, the flexibility of current computational tools make them perfect candidates for use in neural-network based optimization codes, where the CFD simulation results can be used to generate the required response surface [40].

The 2-D Navier-Stokes codes that are used to generate the detailed flowfields seen in most of the published literature are very time consuming to compute and may suffer from convergence problems. To reduce computing time while still retaining the essential physics of the flow field, a number of quasi-one-dimensional differential models have been proposed for ejectors. An interesting approach was taken by Han et al. [38] who used a differential control volume for the secondary stream, while modeling the effects of the primary stream as source terms. The formulation is quite elegant but can only simulate a fully mixed ejector. This is because in the solution procedure the primary flow source terms are equally divided between all of the streamwise control volumes. Thus even though good agreement with experimental results was obtained [38], the model cannot actually compute a mixing length. 
Another such model has been proposed by Yungster \& Trefny [70] specifically for NASA's Independent Ramjet Stream (IRS) Cycle. In the IRS cycle, the secondary (air) stream is pre-fueled far upstream of the rocket resulting in a pre-mixed fuel/air mixture. The rocket is then used only as a source of ignition for the secondary stream, and thus mixing between the flows is not required for thrust augmentation. This particular cycle has actually been the subject of several computational studies $[9,65]$, such as tailoring the fuel injection scheme to alter the location of the thermal throat [65], (which was shown to be feasible for a standard ejector [31]). The differential model of Yungster \& Trefny [70] uses separate control volumes for the primary and secondary streams, while neglecting the shear layer since no mixing is assumed to occur. The streamline which divides the two control volumes is calculated with an empirical pressure-based equation (i.e., $A_{\text {stream }} \propto\left(p_{p}-p_{s}\right)$ ). In addition, chemical reactions are modeled using a heat release function specified between two assumed points which signify the start and end of the heat release. This model agreed very well with analytical benchmark tests [70] carried out by the authors (i.e., Rayleigh flow), but it has not yet been compared to Navier-Stokes based simulations or experimental results.

Analyses of a more fundamental nature, in the form of the basic integral conservation laws have also been applied to ejectors. The goal in this case is to establish fundamental operating trends as well as to identify the dimensionless parameters which govern ejector performance $(\alpha, \zeta, \theta$, etc.). Such treatments generally employ the Inviscid, quasi-one-dimensional conservation laws (mass, momentum, energy) applied to a straight wall mixing duct, subject to specific assumptions about how the two streams initially interact. The majority of the theoretical treatments on ejectors further assume that the two streams fully mix, which essentially represents the ideal case, or the maximum performance that a given ejector configuration can obtain. Analysis under non-ideal, partially-mixed conditions are limited to the work of Papamoschou [57]. In his analysis, conservation laws are applied separately to each 
stream (primary \& secondary) while the boundary between them is calculated assuming pressure continuity $\left(p_{p}=p_{s}\right)$. Effects of mixing were included in the equations as a shear stress calculated by using empirical relations. Although the approach is unique it is only valid for cases when the stream pressures are matched (which may not be the case initially).

For fully mixed theories, an approach taken by many authors $[6,22-24,28,30]$ is to assume that the secondary stream chokes in the aerodynamic throat formed by the high pressure, expanding primary flow. The main advantage of this approach is that it removes the need for specifying conditions at the ejector exit (such as static pressure). Another technique is to assume that the pressures of the primary and secondary streams are matched $[2,36]$ which results in a quadratic equation for the exit Mach number. One can also remove the need for assuming any kind of specific interaction between the two streams by simply specifying a static pressure at the ejector exit [27]. This methodology is the most general, and encompasses the entire operating range of the ejector. In fact, simply by choosing a specific mixedflow pressure, one can encompass the operating ranges of the previously mentioned theories.

There have also been attempts to include the effects of viscosity and incomplete mixing, which can become important especially if the secondary mass flow rates are small [12]. For example, Dutton \& Carrol [22] introduce an empirical pressure recovery coefficient which accounts for losses as well as incomplete mixing. Although this was a simple addition to their fully mixed integral theory, the value of this parameter was dependent on the availability of experimental results. A more rigorous extension of the quasi-one-dimensional integral technique has been proposed by Chow \& Addy [12]. In their work, the authors applied a constant pressure turbulent jet mixing methodology to an ejector. The method is fairly complex, and involves applying the integral conservation laws with an assumed velocity profile, which is a function of 
various empirical parameters related to the mixing of the two jets.

The effects of combustion have also been incorporated in several of the integralbased theories in open literature. In a paper by Han et al. [36], combustion kinetics were treated very simply by adding a source term to the energy equation. A similar approach was adopted by Dobrowolski [20], who used the analysis to theoretically establish that DAB operation is more efficient than SMC. What sets his work apart from the rest, is that his analysis included the effects of a variable area mixing duct by integrating the Crocco Pressure-Area Relation [20] and using it in the momentum equation. The relation has a power law form, and was originally used for single stream devices, such as ramjets. A more detailed treatment of the mixing and combustion processes in an ejector was presented by Peters et al. [58]. Their work was more focused on modeling the mixing process using a modification of the model of Chow \& Addy [12]. However, in contrast to the previously mentioned approaches, the authors invoked chemical equilibrium relations to include the effects of combustion.

\section{$1.3 \quad$ Objectives}

The goal of the current work will be to present an analysis methodology for an ejector that is simple enough to rapidly examine a large number of configurations and accurate enough for use during the initial design stage. The theory will assume complete mixing and will therefore be used to provide idealized performance estimates for the subsonic flight speed range. What sets the present work apart from the rest is its ability to analyze ejector configurations not covered by the approaches available in open literature and to do so in a more complete manner, while maintaining an equation set which can be solved very quickly. Specifically, the theory that will be presented will focus on an annular rocket configuration since it has been shown to promote better mixing [29] and is thus more likely to be used in an ejector design. 
The performance of an ejector can be improved further by constricting its mixing duct which has been shown to improve the compression ratio [27]. For this reason the formulation will also enable analysis of converging mixing ducts and although one such theory was found in literature it was only applicable for ejectors with a central rocket configuration [20]. The combustion in the ejector under SMC conditions will be treated in detail by using an equilibrium calculation which can account for a large number of species. Finally, a method will be provided to estimate a mixing length as well as any performance losses due to incomplete mixing by modifying the equations for the fully mixed-case. 


\section{Chapter 2}

\section{Non-Reacting Ejector Theory}

The theory that will be presented herein is an extension of that provided by Etele et al. [27] for constant area mixing ducts. The ejector will be analyzed by applying a steady, quasi-one-dimensional, integral-based approach to the control volume depicted in Figure 2.1. Complete mixing at the mixed-flow plane (plane $m$ ) will be assumed and although turbulent and viscous effects can occur inside the control volume, there will be no terms accounting for their effects in the governing equations.

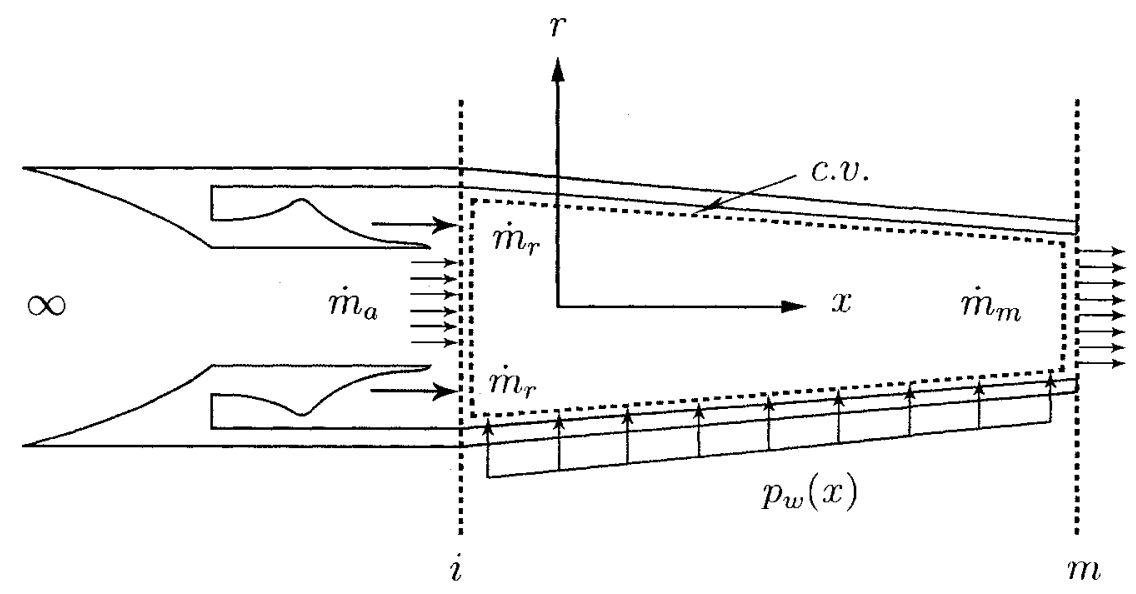

Figure 2.1: Ejector Control Volume 
Considering the mass flow rates shown in Figure 2.1 the conservation of mass can be expressed as,

$$
\dot{m}_{a}+\dot{m}_{r}=\dot{m}_{m}
$$

The mass flow rates in Eq. (2.1) can be more conveniently written in terms of total conditions, to give,

$$
\frac{\dot{m}}{A}=p^{o} \sqrt{\frac{\gamma}{R T^{o}}} \frac{M\left(1+\frac{\gamma-1}{2} M^{2}\right)^{1 / 2}}{\left(1+\frac{\gamma-1}{2} M^{2}\right)^{\frac{\gamma}{\gamma-1}}}
$$

which then simplifies to

$$
\frac{\dot{m}}{A}=\left(\frac{p^{o}}{\sqrt{R T^{\circ}}}\right) \mu
$$

with the functional $\mu$ defined as,

$$
\mu(\gamma, M)=\sqrt{\gamma} M\left(1+\frac{\gamma-1}{2} M^{2}\right)^{\frac{-(\gamma+1)}{2(\gamma-1)}}
$$

Substituting Eq. (2.2) into Eq. (2.1) and re-arranging gives the first equation for the mixed-flow Mach number, $M_{m}$,

$$
\frac{\dot{m}_{r}}{p_{m} A_{m}} \sqrt{\frac{R_{m} T_{m}^{o}}{\gamma_{m}}}(\alpha+1)=M_{m}\left(1+\frac{\gamma_{m}-1}{2} M_{m}^{2}\right)^{1 / 2}
$$

where $\alpha$ is the entrainment ratio and is defined as,

$$
\alpha=\dot{m}_{a} / \dot{m}_{r}
$$

Neglecting viscous forces, the momentum equation for the ejector control volume can be written as follows:

$$
\left(\dot{m}_{a} u_{a}+p_{a} A_{a}\right)+\left(\dot{m}_{r} u_{r}+p_{r} A_{r}\right)-\left(\dot{m}_{m} u_{m}+p_{m} A_{m}\right)-\iint_{0}^{L} p_{w}(x) \cdot \mathrm{d} \vec{S}_{x}=0
$$


The air and rocket momentum terms can be re-expressed using the ideal-gas law and the definition of the speed of sound,

$$
(\dot{m} u+p A)=\dot{m} a\left(M+\frac{p}{\rho u a}\right) A=\dot{m} a\left(M+\frac{1}{\gamma M}\right)
$$

The speed of sound can then be related to sonic conditions in the following manner:

$$
\frac{a}{a^{*}}=\sqrt{\frac{T}{T^{*}}}=\sqrt{\frac{1+\frac{\gamma-1}{2}}{1+\frac{\gamma-1}{2} M^{2}}}=\frac{1}{\sqrt{\frac{2}{\gamma+1}+\left(\frac{\gamma-1}{\gamma+1}\right) M^{2}}}
$$

Using Eq. (2.8) to replace the speed of sound in Eq. (2.7) yields,

$$
\dot{m} a\left(M+\frac{1}{\gamma M}\right)=\dot{m} a^{*} \chi
$$

where $\chi$ is defined as,

$$
\chi(\gamma, M)=\left[\frac{M+\frac{1}{\gamma M}}{\sqrt{\frac{2}{\gamma+1}+\left(\frac{\gamma-1}{\gamma+1}\right) M^{2}}}\right]
$$

Substituting equations (2.7) \& (2.9) into Eq. (2.6) and manipulating the exit momentum flux so as to explicitly maintain the mixed-flow pressure, one obtains,

$$
\dot{m}_{a} a^{*} \chi_{a}+\dot{m}_{r} a_{r}^{*} \chi_{r}-p_{m} A_{m}\left(\gamma M_{m}^{2}+1\right)-\iint_{0}^{L} p_{w}(x) \cdot \mathrm{d} \vec{S}_{x}=0
$$

At sonic conditions, the speed of sound can be expressed as,

$$
a^{* 2}=2 C_{p} \frac{\gamma-1}{\gamma+1} T^{o}
$$


thereby allowing one to write the ratio of the air/rocket sonic sound speeds as,

$$
\frac{a_{a}^{*}}{a_{r}^{*}}=\sqrt{\theta} \Gamma
$$

where $\theta$ and $\Gamma$ have the following definitions:

$$
\begin{gathered}
\theta=\frac{C_{p, a} T_{a}^{o}}{C_{p, r} T_{r}^{o}} \\
\Gamma=\sqrt{\frac{\left(\gamma_{a}-1\right)\left(\gamma_{r}+1\right)}{\left(\gamma_{a}+1\right)\left(\gamma_{r}-1\right)}}
\end{gathered}
$$

One can then invoke the definition of $\alpha$ along with Eq. (2.13) to simplify Eq. (2.11) and yield a second equation for $M_{m}$,

$$
M_{m}^{2}=\frac{1}{\gamma_{m}}\left[\frac{\dot{m}_{r} a_{r}^{*}}{p_{m} A_{m}}\left(\alpha \sqrt{\theta} \Gamma \chi_{a}+\chi_{r}\right)-F_{p, x}-1\right]
$$

where the term $F_{p, x}$ defines the dimensionless wall pressure force,

$$
F_{p, x}=\frac{1}{p_{m} A_{m}} \iint_{0}^{L} p_{w}(x) \cdot \mathrm{d} \vec{S}_{x}
$$

At this point it is convenient to relate the entrainment ratio to other ejector variables by substituting Eq. (2.2) into (2.5) to give,

$$
\alpha=\frac{1}{\zeta}\left(\frac{1-\sigma}{\sigma}\right) \frac{1}{\sqrt{\theta}}\left[\frac{\gamma_{a}\left(\gamma_{r}-1\right)}{\gamma_{r}\left(\gamma_{a}-1\right)}\right]^{1 / 2} \frac{\mu_{a}}{\mu_{r}}
$$

with the following additional definitions:

$$
\sigma=\frac{A_{r}}{A_{i}}
$$




$$
\zeta=\frac{p_{r}^{o}}{p_{a}^{o}}
$$

Since only the $\mu$ functionals depend on the solution variables, $\alpha$ can be more conveniently re-expressed as,

$$
\alpha=\psi \frac{\mu_{a}}{\mu_{r}}
$$

where

$$
\psi=\frac{1}{\zeta}\left(\frac{1-\sigma}{\sigma}\right) \frac{1}{\sqrt{\theta}}\left(\frac{\gamma_{a}\left(\gamma_{r}-1\right)}{\gamma_{r}\left(\gamma_{a}-1\right)}\right)^{1 / 2}
$$

With no heat losses or chemical reactions, the conservation of energy for the ejector simply becomes a conservation of total enthalpy,

$$
\dot{m}_{a} C_{p, a} T_{a}^{o}+\dot{m}_{r} C_{p, r} T_{r}^{o}=\dot{m}_{m} C_{p, m} T_{m}^{o}
$$

Invoking mass conservation and re-arranging gives,

$$
\dot{m}_{r}\left(\frac{\dot{m}_{a}}{\dot{m}_{r}} \frac{C_{p, a} T_{a}^{o}}{C_{p, r} T_{r}^{o}}+1\right)=\dot{m}_{r}\left(\frac{\dot{m}_{a}}{\dot{m}_{r}}+1\right) \frac{C_{p, m} T_{m}^{o}}{C_{p, r} T_{r}^{o}}
$$

The definitions of $\alpha$ and $\theta$ can then be used to simplify the energy equation and give the following result,

$$
T_{m}^{o}=\frac{C_{p, r}}{C_{p, m}}\left(\frac{\alpha \theta+1}{\alpha+1}\right) T_{r}^{o}
$$

Assuming complete mixing at the exit, mass averaging can be used to relate the gas properties to the entrainment ratio,

$$
\begin{gathered}
W_{m}=\frac{1}{\alpha+1}\left(\alpha W_{a}+W_{r}\right) \\
\gamma_{m}=\frac{1}{\alpha+1}\left(\alpha \gamma_{a}+\gamma_{r}\right)
\end{gathered}
$$

Ideal gas relations can then be used to solve for the gas constant and the specific heat 
of the mixture,

$$
\begin{gathered}
R_{m}=\frac{R_{u}}{W_{m}} \\
C_{p, m}=\frac{\gamma_{m} R_{m}}{\gamma_{m}-1}
\end{gathered}
$$

Equations (2.4), (2.16) \& (2.23) represent a system of three equations in the five unknowns, namely $\mu_{a}, \chi_{a}, M_{m}, T_{m}^{o}$ and $F_{p, x}$. However, from equations (2.10) \& (2.3) it is clear that both $\chi_{a}$ and $\mu_{a}$ depend on the air inflow Mach number, $M_{a}$. Thus if one can relate $F_{p, x}$ to other ejector variables, the system will only have three unknowns $\left(M_{a}, M_{m}, T_{m}^{o}\right)$ and can therefore be solved. To implement the solution one needs to know the total inlet conditions $\left(T_{a}^{o}, T_{r}^{o}, p_{r}^{o}, p_{a}^{o}\right)$, the ejector geometry $(\sigma, A(x))$, the rocket exhaust Mach number $M_{r}$, the inlet gas composition $\left(R_{a}, R_{r}, \gamma_{a}, \gamma_{r}\right)$ and the exit pressure $p_{m}$.

\subsection{Wall Pressure Distribution}

To relate the dimensionless wall pressure force to other ejector variables, a wall pressure distribution is required. Since in general, such information will not be known a priori, one has to assume a functional form for $p_{w}(x)$. One can consider two representative cases. The first is the simplest, the case where no additional information about the ejector flowfield is known. This is a linear pressure distribution given by Eq. (2.28) which depends only on the pressure at each end of the ejector. Since the present theory will focus on a configuration with an annular rocket, then the pressure at the ejector inlet will simply be $p_{r}$ while the pressure at the outlet is the mixed-flow pressure $p_{m}$.

$$
p_{w}(x)=p_{r}+\left(p_{m}-p_{r}\right) \frac{x}{L}
$$


The second is slightly more complex and is designed to qualitatively model the expansion-recompression process that occurs inside the ejector. This pressure distribution is given by Eq. (2.29) and depends on the minimum pressure to which the rocket plume expands in addition to the pressure boundary conditions.

$$
p_{w}(x)=p_{\min }+c_{1}\left[c_{2}-\left(\frac{x}{L}-1\right)^{2}\right]^{2}
$$

The constants $c_{1}$ and $c_{2}$ can be found by applying the pressure boundary conditions (i.e., $p_{w}(0)=p_{r}, p_{w}(L)=p_{m}$ ) to give,

$$
\begin{gathered}
c_{2}=(1+\sqrt{\beta})^{-1} \\
c_{1}=\frac{p_{m}-p_{\min }}{c_{2}^{2}}
\end{gathered}
$$

where

$$
\beta=\frac{p_{r}-p_{\min }}{p_{m}-p_{\min }}
$$

The parameter $p_{\min }$ represents the minimum pressure to which the rocket exhaust expands and requires additional equations for its quantification.

\subsection{Solution for Minimum Rocket Pressure}

It is clear at this point that if one wishes to use Eq. (2.29) a way of solving for the parameter $p_{\min }$ is required. Two different methods are presented to solve for the minimum rocket pressure, the first is a Control Volume calculation, while the second one makes use of a Riemann solver. Both methods assume that the air and rocket streams are isentropic and that no mixing takes place before the expansion point. 


\subsubsection{Control Volume Solution}

The Control Volume (CV) approach for $p_{\min }$ treats all inflow conditions as known and is thus solved in conjunction with equations (2.4), (2.16) \& (2.23). The method also assumes quasi-one-dimensional and isentropic flow in both streamtubes. Control volumes for both streams are depicted in Figure 2.2 and extend from the ejector inlet to plane 2 , which represents the plane at which the rocket stream fully expands and reaches its minimum pressure.

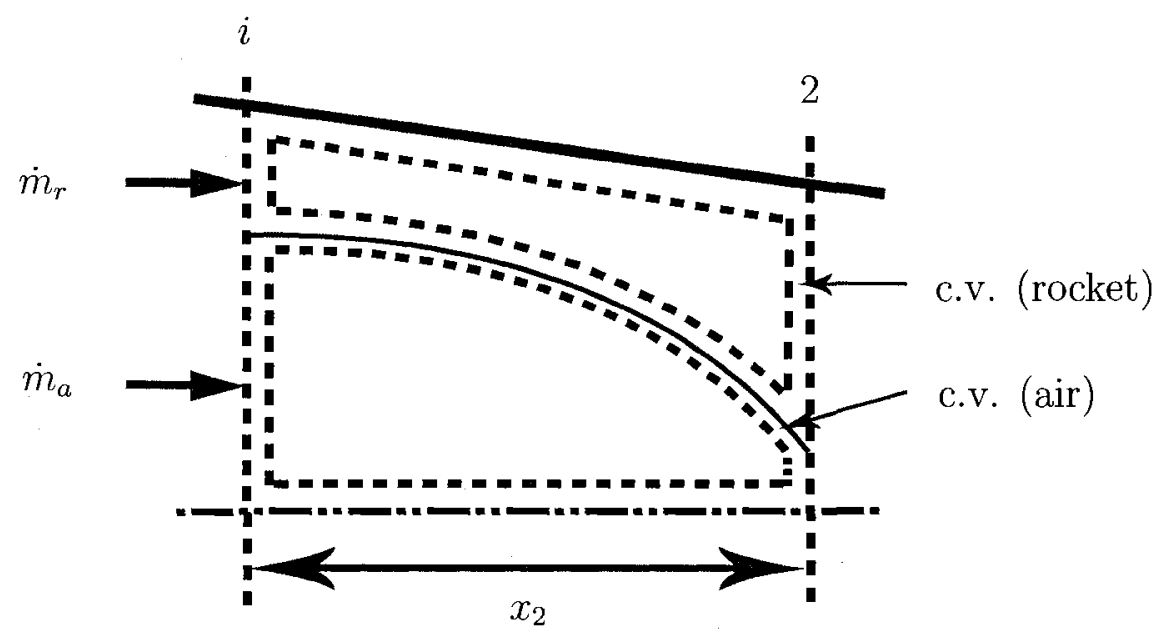

Figure 2.2: Air \& Rocket Control Volumes for Estimating $p_{\min }$

One can then apply momentum conservation to each control volume to give the following set of equations:

$$
\begin{gathered}
-\left(\dot{m}_{a} u_{a}+p_{a} A_{a}\right)_{i}+\left(\dot{m}_{a} u_{a}+p_{a} A_{a}\right)_{2}-\iint_{s l} p_{s l}(x) \cdot \mathrm{d} \vec{S}_{x}=0 \\
-\left(\dot{m}_{r} u_{r}+p_{r} A_{r}\right)_{i}+\left(\dot{m}_{r} u_{r}+p_{m i n} A_{r}\right)_{2}-\iint_{s l} p_{s l}(x) \cdot \mathrm{d} \vec{S}_{x}-\iint_{0}^{x_{2}} p_{w}(x) \cdot \mathrm{d} \vec{S}_{x}=0
\end{gathered}
$$

Adding the two momentum equations to eliminate the shear layer integral $(s l)$ while 
using equations (2.7) \& (2.9) to simplify the momentum fluxes yields,

$$
\dot{m}_{a}\left(\chi_{a, 2} a_{a, 2}^{*}-\chi_{a, i} a_{a, i}^{*}\right)+\dot{m}_{r}\left(\chi_{r, 2} a_{r, 2}^{*}-\chi_{r, i} a_{r, i}^{*}\right)-\iint_{0}^{x_{2}} p_{w}(x) \cdot \mathrm{d} \vec{S}_{x}=0
$$

Since both streams do not mix and are adiabatic, then by Eq. (2.12):

$$
\begin{aligned}
& a_{a, i}^{*}=a_{a, 2}^{*}=a_{a}^{*} \\
& a_{r, i}^{*}=a_{r, 2}^{*}=a_{r}^{*}
\end{aligned}
$$

which allows the definitions of $\alpha, \theta \& \Gamma$ to be used to simplify Eq. (2.35),

$$
\left(\chi_{r, i}-\chi_{r, 2}\right)+\alpha \sqrt{\theta} \Gamma\left(\chi_{a, i}-\chi_{a, 2}\right)-F_{p, x_{2}}=0
$$

where

$$
F_{p, x_{2}}=\frac{1}{\dot{m}_{r} a_{r}^{*}} \iint_{0}^{x_{2}} p_{w}(x) \cdot \mathrm{d} \vec{S}_{x}
$$

The term $F_{p, x_{2}}$ has the same form as Eq. (2.17) but is integrated only up to the point where the rocket stream reaches its minimum pressure. The axial location of this point can be obtained directly from Eq. (2.29) by minimizing the expression to give,

$$
\frac{x_{2}}{L}=1-\sqrt{c_{2}}
$$

Equations (2.29) \& (2.38) can be substituted into the expression for $F_{p, x_{2}}$ and integrated to obtain a closed-form expression (Eq. (2.85)) in terms of the constants $c_{1}$ $\& c_{2}$. Since both of these constants are functions of $p_{\min }$ (see Eq's (2.31), (2.30), $(2.32))$, the dimensionless pressure integral $\left(F_{p, x_{2}}\right)$ becomes a function of the solution variable, $p_{\min }$. 
Next, the conservation of mass is applied to the air control volume in conjunction with Eq. (2.2) to obtain,

$$
A_{a, i} \frac{p_{a, i}^{o}}{\sqrt{R_{a} T_{a, i}^{o}}} \mu_{a, i}=A_{a, 2} \frac{p_{a, 2}^{o}}{\sqrt{R_{a} T_{a, 2}^{o}}} \mu_{a, 2}
$$

Since the flow is both isentropic and adiabatic, the above equation reduces to,

$$
A_{a, i} \mu_{a, i}=A_{a, 2} \mu_{a, 2}
$$

Applying the same approach to the rocket stream gives,

$$
A_{r, i} \mu_{r, i}=A_{r, 2} \mu_{r, 2}
$$

Given the fact that the flow areas at station 2 are constrained,

$$
A_{r, 2}+A_{a, 2}=A\left(x_{2}\right)
$$

allows equations $(2.40),(2.41)$ and $(2.42)$ to be combined into an expression in terms of the air/rocket Mach numbers only,

$$
A_{a, i} \frac{\mu_{a, i}}{\mu_{a, 2}}+A_{r, i} \frac{\mu_{r, i}}{\mu_{r, 2}}=A\left(x_{2}\right)
$$

Since both streams were assumed isentropic, $p_{\min }$ can be related to $M_{r, 2}$ with the isentropic expression for total pressure which can be written as,

$$
p_{\min }=p_{r}^{o}\left(1+\frac{\gamma_{r}-1}{2} M_{r, 2}^{2}\right)^{\frac{-\gamma_{r}}{\gamma_{r}-1}}
$$

The derivation thus yields two equations, $(2.36) \&(2.43)$ in the unknowns $\mu_{a, 2}, \chi_{a, 2}$, $\mu_{r, 2}, \chi_{r, 2}$ and $p_{\min }$. If one invokes the definitions of $\mu$ and $\chi$ as well as Eq. (2.44), the 
unknowns are reduced to the air and rocket Mach numbers, $M_{a, 2}$ and $M_{r, 2}$. It should be noted that since the solution for $p_{\min }$ requires knowledge of the ejector inflow conditions $\left(M_{a}\right)$, it must be repeated at each iteration of the solution of equations (2.4), (2.16) \& (2.23).

\subsubsection{Riemann Solution}

The Riemann approach is quite different from the one presented above with the basic features shown in Figure 2.3. In the case of the ejector, an analogy can be drawn between the expansion of the rocket plume and a classic shock tube problem. The problem consists of a one-dimensional flow where two different gases at different states are initially separated by a diaphragm which is then burst. When this happens, a shock travels into the driven (lower pressure) gas and an expansion wave travels into the driver gas. The Riemann problem essentially involves solving for the various flow variables at the moving gas interface which forms between the two gases. A more detailed discussion of shock tubes and the Riemann problem is outlined by Gottlieb \& Groth [35].

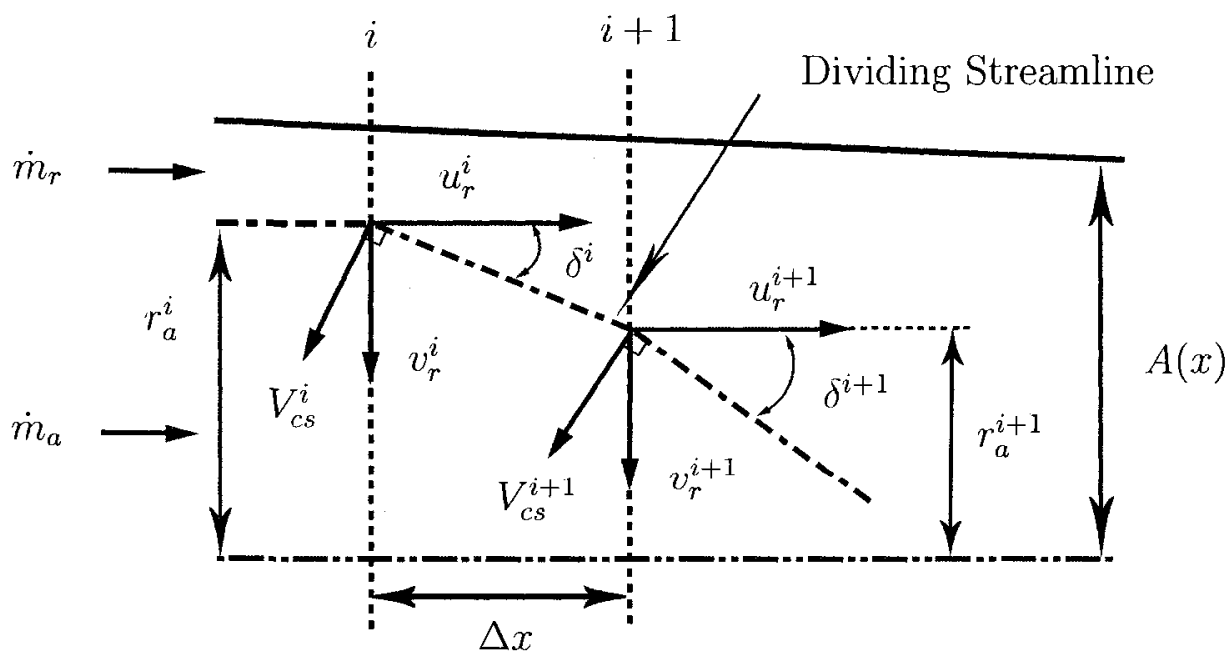

Figure 2.3: Riemann Problem Set-Up Adapted to Ejector 
Referring to Fig. 2.3, the method is set up such that the radial motion of the rocket plume expanding against the air stream at some location $x$ is treated as a shock tube. For the case when the rocket pressure exceeds the static pressure of the air, the rocket plume becomes the driver gas. Since the streams are assumed not to mix, one can define a contact surface, or more generally a dividing streamline which separates the two streams. In the present case, a Riemann solver is used to solve for the velocity of the contact surface which along with the streamwise velocity can be used to trace out the dividing streamline. The streamline would then define the area distribution of the streamtubes which surround each stream at which point isentropic relations can be used to find the remaining flow variables. The present formulation uses a Riemann solver proposed by Gottlieb \& Groth [35] to solve for $V_{c s}$.

First, from the Mach numbers of the rocket and air streams the sonic reference areas can be found using the Area-Mach Number Relation [3].

$$
f\left(\frac{A}{A^{*}}\right)=\frac{1}{M^{2}}\left(\frac{2}{\gamma+1}+\frac{\gamma-1}{\gamma+1} M^{2}\right)^{\frac{\gamma+1}{\gamma-1}}=\left(\frac{A}{A^{*}}\right)^{2}
$$

Next the Riemann solver is applied to find the velocity of the contact surface at node $i$.

$$
V_{c s}^{i}=f\left(p_{r}^{i}, p_{a}^{i}, \rho_{r}^{i}, \rho_{a}^{i}, R_{r}, R_{a}, \gamma_{r}, \gamma_{a}\right)
$$

To define the streamline at the next node, the radial velocity component at node $i+1$ is required which can be written as,

$$
v_{r}^{i+1}=v_{r}^{i}+\Delta v_{r}
$$

Referring to the velocity polygon shown in Fig. 2.4 one can express $\Delta v_{r}$ as,

$$
\Delta v_{r}=V_{c s}^{i} \cos \left(\delta_{i}\right)=\frac{V_{c s}^{i} u_{r}^{i}}{\sqrt{\left[u_{r}^{i}\right]^{2}+\left[v_{r}^{i}\right]^{2}}}
$$




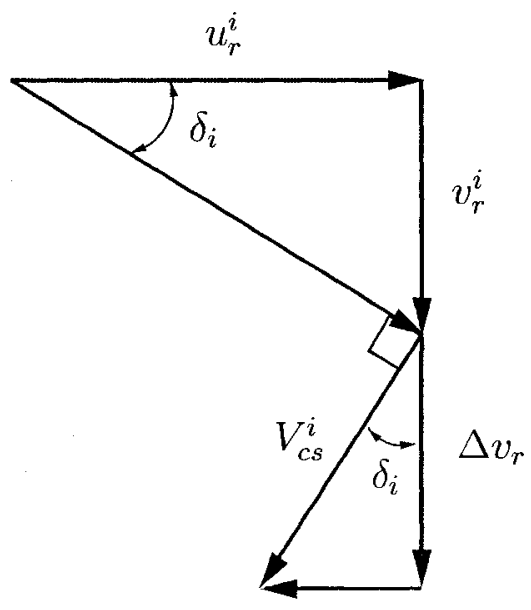

Figure 2.4: Velocity Polygon at Node $i$

One can then substitute Eq. (2.48) into Eq. (2.47) to obtain,

$$
v_{r}^{i+1}=v_{r}^{i}+\frac{V_{c s}^{i}}{\sqrt{1+\left(\frac{v_{r}^{i}}{u_{r}^{i}}\right)^{2}}}
$$

To define the dividing streamline the following relationship is used,

$$
\frac{\mathrm{d} r}{\mathrm{~d} x}=\frac{v_{r}}{u_{r}}
$$

which simply states that the velocity vector is tangent to the streamline. To apply the above equation, forward differencing is used for discretization to yield,

$$
r_{a}^{i+1}=r_{a}^{i}-\Delta x \frac{v_{r}^{i+1}}{u_{r}^{i}}
$$

while the flow areas can be found using,

$$
\begin{gathered}
A_{a}^{i+1}=\pi\left(r_{a}^{i+1}\right)^{2} \\
A_{r}^{i+1}=A(x+\Delta x)-A_{a}^{i+1}
\end{gathered}
$$


With the flow areas known, the Area-Mach Number Relation can be used to solve for the Mach number,

$$
M_{j}^{i+1}=f\left(\frac{A_{j}^{i+1}}{A_{j}^{*}}\right) \quad j=a, r
$$

after which isentropic relations along with the ideal gas law are used to solve for the state of each stream at $i+1$,

$$
\begin{gathered}
T_{j}^{i+1}=T_{j}^{o}\left[1+\frac{\gamma_{j}-1}{2}\left(M_{j}^{i+1}\right)^{2}\right]^{-1} j=a, r \\
p_{j}^{i+1}=p_{j}^{o}\left[1+\frac{\gamma_{j}-1}{2}\left(M_{j}^{i+1}\right)^{2}\right]^{\frac{-\gamma_{j}}{\gamma_{j}-1}} \quad j=a, r \\
\rho_{j}^{i+1}=\frac{p_{j}^{i+1}}{R_{j} T_{j}^{i+1}} \quad j=a, r
\end{gathered}
$$

Lastly, mass conservation can be used to solve for the axial velocity,

$$
u_{j}^{i+1}=\frac{\dot{m}_{j}}{\rho_{j}^{i+1} A_{j}^{i+1}} \quad j=a, r
$$

The procedure is then marched in the $x$-direction until $v_{r} \leq 0$ (the expansion stops). One thing to note regarding the above procedure, is that the air stream can choke while $v_{r}>0$. This type of situation would not happen in reality, since the mass flow rate of the air at the inlet would adjust to accommodate a smaller $A_{a}^{*}$. However, because $p_{\min }$ is solved at each iteration of the ejector solution, equations (2.4), (2.16) \& (2.23) govern the mass flow rate of the air, and not the Riemann solver. Essentially, the type of feedback required to allow the air flow to adjust is not possible to implement in the present formulation. Thus to be able to solve for $p_{\min }$ when the air flow chokes, a slight modification to the solution process is made. If critical conditions are encountered in the air stream during a given run while the streamline is still expanding, the Riemann procedure is stopped. At the point where the solver stops, it is then assumed that $A_{a}=A_{a}^{*}$. This fixes the area of the rocket 
stream (by Eq. (2.42)), and allows one to use Eq. (2.45) to find the Mach number at that station, from which $p_{\min }$ can be found using Eq. (2.56).

\subsection{Wall Pressure Integral}

The integral in Eq. (2.17) is a surface integral and needs to be simplified before it can be used in the solution procedure. One can actually convert the surface integral to a double integral by considering the three-dimensional shape of the ejector control volume shown in Figure 2.5.

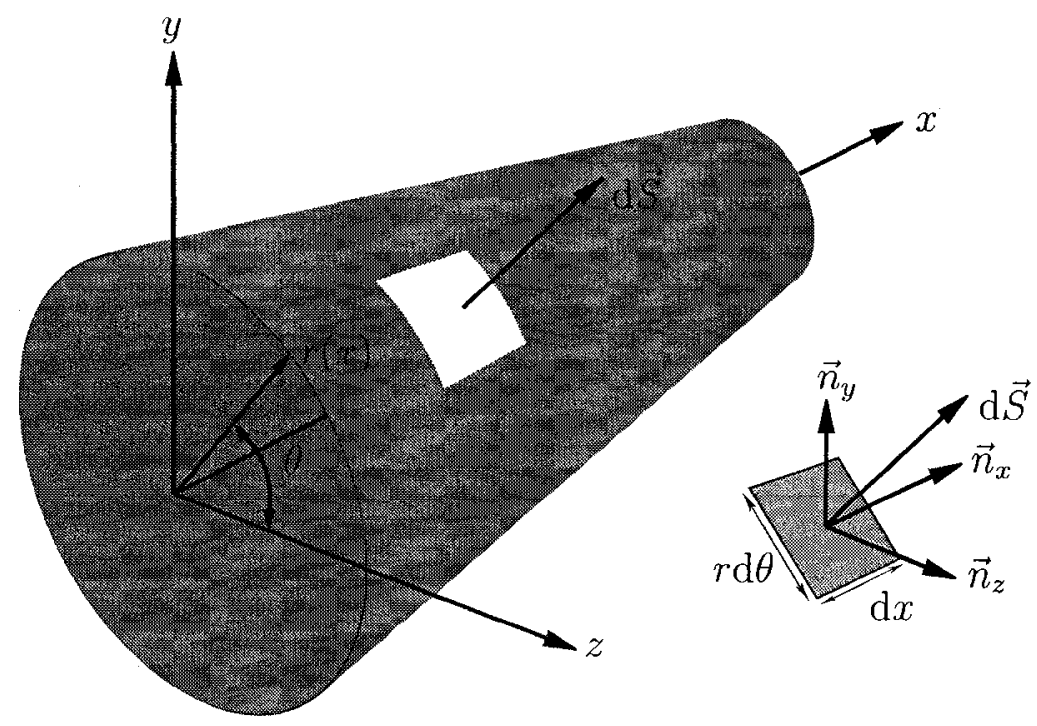

Figure 2.5: Ejector Control Volume showing surface element $\mathrm{d} \vec{S}$

The equation for the surface depicted in the figure can be expressed as,

$$
f_{c v}=z^{2}+y^{2}-[r(x)]^{2}=0
$$

By definition, a vector normal to any surface can be found by taking the gradient of the equation which defines that surface. Doing so for the control volume surface 
yields the normal vector $\vec{n}$ as,

$$
\vec{n}=\nabla f=-2 r(x) \frac{\mathrm{d} r}{\mathrm{~d} x} \hat{i}+2 y \hat{j}+2 z \hat{k}
$$

At this point it is convenient to decompose the elemental surface $\mathrm{d} \vec{S}$ in the following manner:

$$
\mathrm{d} \vec{S}_{x}=\frac{\vec{n}_{x}}{\left\|\vec{n}_{x}\right\|} r \mathrm{~d} \theta \mathrm{d} x
$$

where only the axial component is considered, since that is the component of the wall pressure force that is included in the momentum balance. One can now make use of Eq. (2.60) to obtain the $x$-component of the unit normal vector,

$$
\hat{n}_{x}=\left(\frac{\nabla f}{\|\nabla f\|}\right) \cdot \hat{i}=\frac{-2 r(x) \mathrm{d} r / \mathrm{d} x}{2 \sqrt{[r(x)]^{2}(\mathrm{~d} r / \mathrm{d} x)^{2}+y^{2}+z^{2}}} \cdot \hat{i}
$$

Using Equation (2.59) to simplify the above expression gives the unit vector as,

$$
\hat{n}_{x}=-\frac{\mathrm{d} r / \mathrm{d} x}{\sqrt{1+(\mathrm{d} r / \mathrm{d} x)^{2}}}
$$

Substituting Eqs. (2.62) \& (2.61) into Eq. (2.17), transforms the surface integral into a double integral,

$$
F_{p, x}=-\frac{1}{p_{m} A_{m}} \int_{0}^{L} \int_{0}^{2 \pi} \frac{p_{w}(x) r(x) \mathrm{d} r / \mathrm{d} x}{\sqrt{1+(\mathrm{d} r / \mathrm{d} x)^{2}}} d \theta \mathrm{d} x
$$

Since all of the terms inside the integral do not vary in the circumferential direction, the above expression can be reduced further to give the final result,

$$
F_{p, x}=-\frac{2 \pi}{p_{m} A_{m}} \int_{0}^{L} \frac{p_{w}(x) r(x) \mathrm{d} r / \mathrm{d} x}{\sqrt{1+(\mathrm{d} r / \mathrm{d} x)^{2}}} \mathrm{~d} x
$$




\subsection{Numerical Solution}

The numerical solution for the constricted ejector is significantly more involved than the unconstricted case due to the fact that a separate solution procedure is required to quantify the pressure distribution (CV or Riemann) which then needs to be integrated. The equations which govern the ejector consist of a linear equation for $T_{m}^{o}$ (Eq. (2.23)) as well as a pair of non-linear equations for $M_{a} \& M_{m}$ (Eq.s (2.4) and (2.16) respectively). The first step is to convert the non-linear equations into a form better suited to the solution process by substituting Eq. (2.20) into Eqs. (2.4) \& (2.16) to give,

$$
\begin{gathered}
f_{1}=-\gamma_{m} M_{m}^{2}+\left[\frac{\psi \dot{m}_{r} a_{r}^{*}}{\mu_{r} p_{m} A_{m}} \sqrt{\theta} \Gamma\right] \mu_{a} \chi_{a}+\left[\frac{\dot{m}_{r} a_{r}^{*}}{p_{m} A_{m}} \chi_{r}-F_{p, x}-1\right]=0 \\
f_{2}=\frac{\dot{m}_{r}}{p_{m} A_{m}} \sqrt{\frac{R_{m} T_{m}^{o}}{\gamma_{m}}}\left(\psi \frac{\mu_{a}}{\mu_{r}}+1\right)-M_{m}\left(1+\frac{\gamma_{m}-1}{2} M_{m}^{2}\right)^{1 / 2}=0
\end{gathered}
$$

To solve the non-linear system one can make use of the Newton-Raphson method. The method essentially linearizes the equations, which then allows the system to be iterated toward the final solution starting from an initial guess. Applying the method to equations (2.64) and (2.65) and writing the linearized system in matrix form yields,

$$
\left[\begin{array}{cc}
\frac{\partial f_{1}}{\partial M_{a}} & \frac{\partial f_{1}}{\partial M_{m}} \\
\frac{\partial f_{2}}{\partial M_{a}} & \frac{\partial f_{2}}{\partial M_{m}}
\end{array}\right]^{k}\left[\begin{array}{c}
\Delta M_{a} \\
\Delta M_{m}
\end{array}\right]^{k+1}=-\left[\begin{array}{l}
f_{1} \\
f_{2}
\end{array}\right]^{k}
$$

where $k$ is the current iteration level. The matrix on the left hand side is the system Jacobian and contains the derivatives of the functions $f_{1}$ and $f_{2}$ with respect to the solution variables. To solve Eq. (2.66) one needs to define all of the system derivatives. Differentiating the first functional with respect to the solution variables $M_{a}$ and $M_{m}$ 
gives,

$$
\begin{gathered}
\frac{\partial f_{1}}{\partial M_{a}}=\frac{\psi \sqrt{\theta} \Gamma \dot{m}_{r} a_{r}^{*}}{\mu_{r} p_{m} A_{m}}\left[\mu_{a} \frac{\partial \chi_{a}}{\partial M_{a}}+\chi_{a} \frac{\partial \mu_{a}}{\partial M_{a}}\right] \\
\frac{\partial f_{1}}{\partial M_{m}}=-2 \gamma_{m} M_{m}
\end{gathered}
$$

In a similar manner one can also obtain derivatives of the second functional to yield,

$$
\frac{\partial f_{2}}{\partial M_{m}}=-\left(1+\frac{\gamma_{m}-1}{2} M_{m}^{2}\right)^{1 / 2}\left[\frac{\left(\gamma_{m}-1\right) M_{m}^{2}}{2\left(1+\frac{\gamma_{m}-1}{2} M_{m}^{2}\right)}+1\right]
$$

Keeping in mind that $T_{m}^{o}$ depends on $M_{a}$ (through $\alpha$ ) the derivative of Eq. (2.65) with respect to $M_{a}$ can be obtained as,

$$
\frac{\partial f_{2}}{\partial M_{a}}=\frac{\dot{m}_{r}}{p_{m} A_{m}} \sqrt{\frac{R_{m} T_{m}^{o}}{\gamma_{m}}}\left[\frac{\psi}{\mu_{r}} \frac{\partial \mu_{a}}{\partial M_{a}}+\frac{(\alpha+1)}{2 T_{m}^{o}} \frac{\partial T_{m}^{o}}{\partial M_{a}}\right]
$$

while the derivative of $T_{m}^{o}$ can be acquired from the energy equation, giving,

$$
\frac{\partial T_{m}^{o}}{\partial M_{a}}=\frac{C_{p, r}}{C_{p, m}} T_{r}^{o} \frac{\partial \mu_{a}}{\partial M_{a}}\left[\frac{(\theta-1)}{(\alpha+1)^{2}}\right] \frac{\psi}{\mu_{r}}
$$

Lastly, the derivatives of the $\mu$ and $\chi$ functionals are required since they appear several times. To obtain these one can simply differentiate Eqs. (2.3) and (2.10) to give the following relations:

$$
\begin{gathered}
\frac{\partial \chi}{\partial M}=\left(\frac{2}{\gamma+1}+\frac{\gamma-1}{\gamma+1} M^{2}\right)^{-1 / 2}\left[\left(1-\frac{1}{\gamma M^{2}}\right)\right. \\
\left.-\left(M^{2}+\frac{1}{\gamma}\right)\left(\frac{\gamma-1}{\gamma+1}\right)\left(\frac{2}{\gamma+1}+\frac{\gamma-1}{\gamma+1} M^{2}\right)^{3}\right] \\
\frac{\partial \mu}{\partial M}=\sqrt{\gamma}\left[\left(1+\frac{\gamma-1}{2} M^{2}\right)^{\frac{-(\gamma+1)}{2(\gamma-1)}}-\frac{M^{2}}{2}(\gamma+1)\left(1+\frac{\gamma-1}{2} M^{2}\right)^{\frac{(1-3 \gamma)}{2(\gamma-1)}}\right]
\end{gathered}
$$


When Eq. (2.66) is solved at a given iteration level one can then update the values of the solution variables in the following manner:

$$
\begin{gathered}
M_{a}^{k+1}=M_{a}^{k}+\left(\Delta M_{a}\right)^{k} \\
M_{m}^{k+1}=M_{m}^{k}+\left(\Delta M_{m}\right)^{k}
\end{gathered}
$$

The solution is considered converged when the right hand side of Eqs. (2.64) and (2.65) are sufficiently close to zero. For the present computations the tolerance is set to,

$$
\left|f_{1}, f_{2}\right| \leq 1 \times 10^{-7}
$$

A flowchart representation of the solution process is shown in Figure 2.6. When the initial guesses for $M_{a}$ and $M_{m}$ are set to 0.2 and 0.8 respectively, a converged solution can be obtained in less than 15 iterations.

Several options exist for computing $F_{p, x}$ with the choice coming down to what pressure distribution is used. In either case, the same linear nozzle contour function will be used whose profile can be expressed as,

$$
r(x)=r_{i}+\left(\frac{\mathrm{d} r}{\mathrm{~d} x}\right) x
$$

When the pressure distribution is defined by Eq. (2.29), one needs to solve for $p_{\min }$ before $F_{p, x}$ can be computed. This means that either the CV or Riemann solution is implemented first using the value of $M_{a}$ at the given iteration level. Once $p_{\min }$ is known, one can integrate Equation (2.63) to obtain a closed form expression for $F_{p, x}$. Accordingly, Eqs. (2.29) \& (2.76) are substituted into Eq. (2.63) and integrated, 


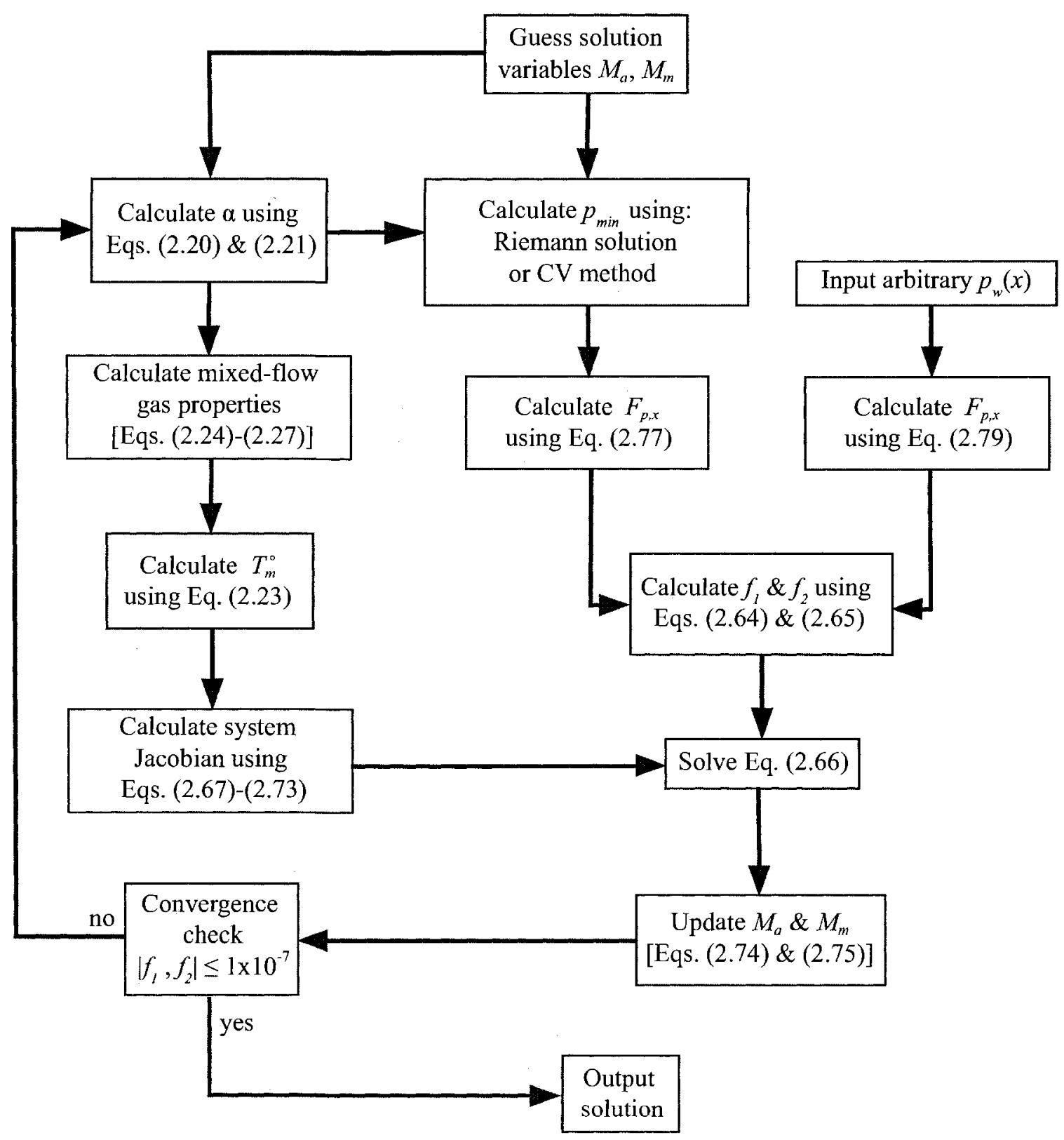

Figure 2.6: Ejector Solution Process (Non-Reacting) 
giving,

$$
F_{p, x}=\frac{-2 \pi L \frac{\mathrm{d} r}{\mathrm{~d} x}}{p_{m} A_{m} \sqrt{1+\left(\frac{\mathrm{d} r}{\mathrm{~d} x}\right)^{2}}}\left[\frac{p_{m}}{2}\left(2 r_{i}+r_{m}\right)-\frac{c_{1} c_{2}}{6}\left(4 r_{i}+r_{m}\right)+\frac{c_{1}}{30}\left(6 r_{i}+r_{m}\right)\right]
$$

which is specific to the polynomial-based pressure distribution. If one wishes to use an arbitrary $p_{w}(x)$ the most convenient method of solution is numerical integration. The second-order accurate trapezoidal rule will be used in the present case which can be expressed for an arbitrary function as,

$$
\int_{a}^{b} f(x) \mathrm{d} x \approx \Delta x\left[\frac{f(b)-f(a)}{2}+\sum_{i=1}^{n} f\left(x_{i}\right)\right]
$$

Applying the above method to equation (2.63) gives the following numerical formula:

$$
F_{p, x} \approx \frac{-2 \pi \Delta x}{p_{m} A_{m}}\left[\frac{K(L)-K(0)}{2}+\sum_{i=1}^{n} K\left(x_{i}\right)\right]
$$

where

$$
K(x)=\frac{p_{w}(x)}{\sqrt{1+(\mathrm{d} r / \mathrm{d} x)^{2}}} r(x) \frac{\mathrm{d} r}{\mathrm{~d} x}
$$

while centered differencing can be used to evaluate the derivative $\mathrm{d} r / \mathrm{d} x$,

$$
\frac{\mathrm{d} r}{\mathrm{~d} x} \approx \frac{r_{i+1}-r_{i-1}}{2 \Delta x}
$$

\subsubsection{Method - Numerical Solution}

The solution of the equations which govern $p_{\min }$ is carried out using the NewtonRaphson method. The procedure itself is analogous to the method used for Eqs. (2.4) and (2.16) since both equation sets contain a wall pressure integral. In this case, Eq. (2.20) can once again be used to rewrite Eqs. (2.36) and (2.43) in the 
following form:

$$
\begin{gathered}
f_{1, C V}=\left(\chi_{r, i}-\chi_{r, 2}\right)+\psi \sqrt{\theta} \Gamma\left(\frac{\mu_{a, i}}{\mu_{r, i}}\right)\left(\chi_{a, i}-\chi_{a, 2}\right)-F_{p, x_{2}}=0 \\
f_{2, C V}=A_{a, i} \frac{\mu_{a, i}}{\mu_{a, 2}}+A_{r, i} \frac{\mu_{r, i}}{\mu_{r, 2}}-A\left(x_{2}\right)=0
\end{gathered}
$$

where the term $A\left(x_{2}\right)$ is calculated from the radial contour of the nozzle defined by equation (2.76). To define the Jacobian, one can differentiate equations (2.81) and (2.82) with respect to the solution variables $\left(M_{r 2}, M_{a 2}\right)$ to obtain,

$$
\begin{aligned}
\frac{\partial f_{1, C V}}{\partial M_{r, 2}}=-\frac{\partial \chi_{r, 2}}{\partial M_{r, 2}} & \frac{\partial f_{1, C V}}{\partial M_{a, 2}}=-\alpha \sqrt{\theta} \Gamma \frac{\partial \chi_{a, 2}}{\partial M_{a, 2}} \\
\frac{\partial f_{2, C V}}{\partial M_{r, 2}}=-A_{r, i} \frac{\mu_{r, i}}{\mu_{r, 2}^{2}} \frac{\partial \mu_{r, 2}}{\partial M_{r, 2}} & \frac{\partial f_{2, C V}}{\partial M_{a, 2}}=-A_{a, i} \frac{\mu_{a, i}}{\mu_{a, 2}^{2}} \frac{\partial \mu_{a, 2}}{\partial M_{a, 2}}
\end{aligned}
$$

Solution of the above system also requires that $F_{p, x_{2}}$ be related to the solution variables. Since $p_{\min }$ is found only for the polynomial pressure distribution (Eq. (2.29)), it is worthwhile to obtain a closed form expression for $F_{p, x_{2}}$ as well. Carrying out the integration gives,

$$
\begin{aligned}
F_{p, x_{2}}= & \frac{-2 \pi \frac{\mathrm{d} r}{\mathrm{~d} x}}{\dot{m}_{r} a_{r}^{*} \sqrt{1+\left(\frac{\mathrm{d} r}{\mathrm{~d} x}\right)^{2}}}\left[p_{\min } x_{2}\left(r_{i}+\frac{x_{2}}{2} \frac{\mathrm{d} r}{\mathrm{~d} x}\right)+r_{i} c_{1} L\left(c_{2}^{2}-\frac{2}{3} c_{2}\right.\right. \\
& \left.\left.-\frac{8}{15} c_{2}^{5 / 2}+\frac{1}{5}\right)+\frac{\mathrm{d} r}{\mathrm{~d} x} c_{1} L^{2}\left(\frac{c_{2}^{2}}{2}-\frac{c_{2}}{6}+\frac{1}{30}-\frac{8}{15} c_{2}^{5 / 2}+\frac{c_{2}^{3}}{6}\right)\right]
\end{aligned}
$$

where $x_{2}$ is defined by Eq. (2.38). To ensure that the solution process remains stable, one can introduce a damping factor, $\Upsilon_{C V}$ as,

$$
\begin{aligned}
& M_{a, 2}^{k+1}=M_{a, 2}^{k}+\Upsilon_{C V}\left[\Delta M_{a, 2}\right]^{k} \\
& M_{r, 2}^{k+1}=M_{r, 2}^{k}+\Upsilon_{C V}\left[\Delta M_{r, 2}\right]^{k}
\end{aligned}
$$


Although more rigorous methods are available for choosing a value for $\Upsilon_{C V}$, a value of 0.1 was found to work well. The damping is conditional and is applied only if the following condition is met:

$$
\left\|\left(f_{1, C V}, f_{2, C V}\right)\right\|^{k+1} \geq\left\|\left(f_{1, C V}, f_{2, C V}\right)\right\|^{k}
$$

which is simply a mathematical condition that represents the solution diverging. The convergence tolerance in this case is set to,

$$
\left|f_{1, C V}, f_{2, C V}\right| \leq 1 \times 10^{-7}
$$

The solution process for $p_{\min }$ is summarized in Figure 2.7 and is invoked at each iteration level of the solution for the entire ejector (see Fig. 2.6). This means that a converged solution for $p_{\min }$ is obtained at every iteration of the overall solution process. The initial guesses for $M_{a, 2}$ and $M_{r, 2}$ are set to $M_{a}$ and $1.2 \times M_{r}$ respectively and yield a converged solution in less than 220 iterations. 
Chapter 2. Non-Reacting Ejector Theory

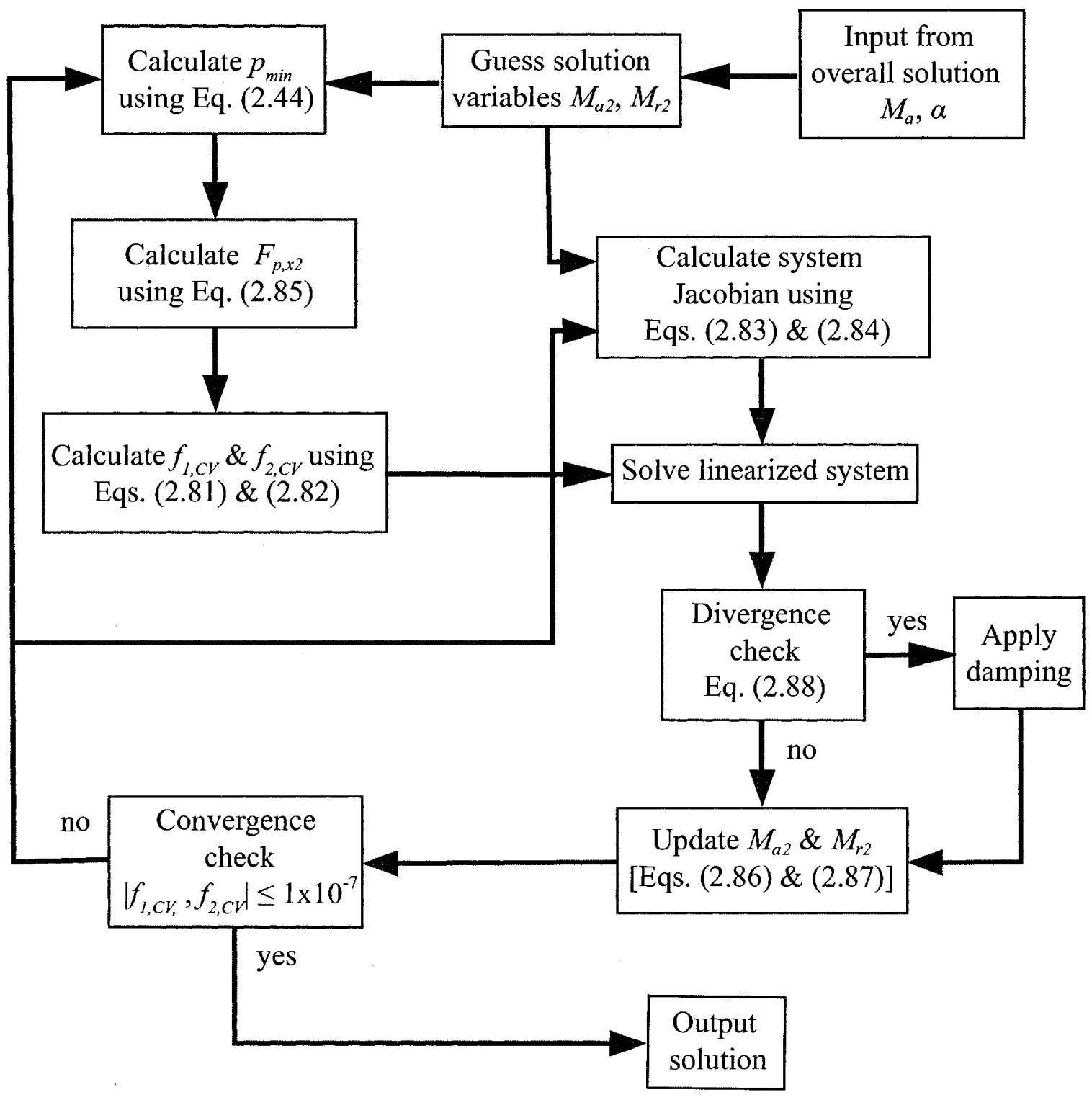

Figure 2.7: CV-based Solution Process for $p_{\min }$ 


\subsubsection{Area-Mach Number Relation}

Solving for $p_{\min }$ using the Riemann solver, although already described, requires the solution of the Area-Mach Number Relation. Since a single non-linear equation is now being dealt with, application of the Newton-Raphson method is fairly straightforward. If one rewrites the Area-Mach Number Relation as,

$$
f_{A M}=\frac{1}{M^{2}}\left[\frac{2}{\gamma+1}+\frac{\gamma-1}{\gamma+1} M^{2}\right]^{\frac{\gamma+1}{\gamma-1}}-\left(\frac{A}{A^{*}}\right)^{2}
$$

then linearizing it with the Newton-Raphson method would yield,

$$
M^{k+1}=M^{k}-\left[\frac{f_{A M}}{\frac{\partial f_{A M}}{\partial M}}\right]^{k}
$$

where $\frac{\partial f_{A M}}{\partial M}$ is given as,

$$
\frac{\partial f_{A M}}{\partial M}=2\left(\frac{A}{A^{*}}\right)^{2}\left[M\left(\frac{2}{\gamma+1}+\frac{\gamma-1}{\gamma+1} M^{2}\right)^{-1}-\frac{1}{M}\right]
$$

The initial guess for $M$ is passed from the Riemann solver and is simply the Mach number at the previous node (within the Riemann solution). The convergence tolerance is set to,

$$
\left|f_{A M}\right| \leq 1 \times 10^{-7}
$$

and yields a converged solution in less than 10 iterations. The process is shown schematically in Figure 2.8 and is invoked at each solution point within the Riemann solver. 


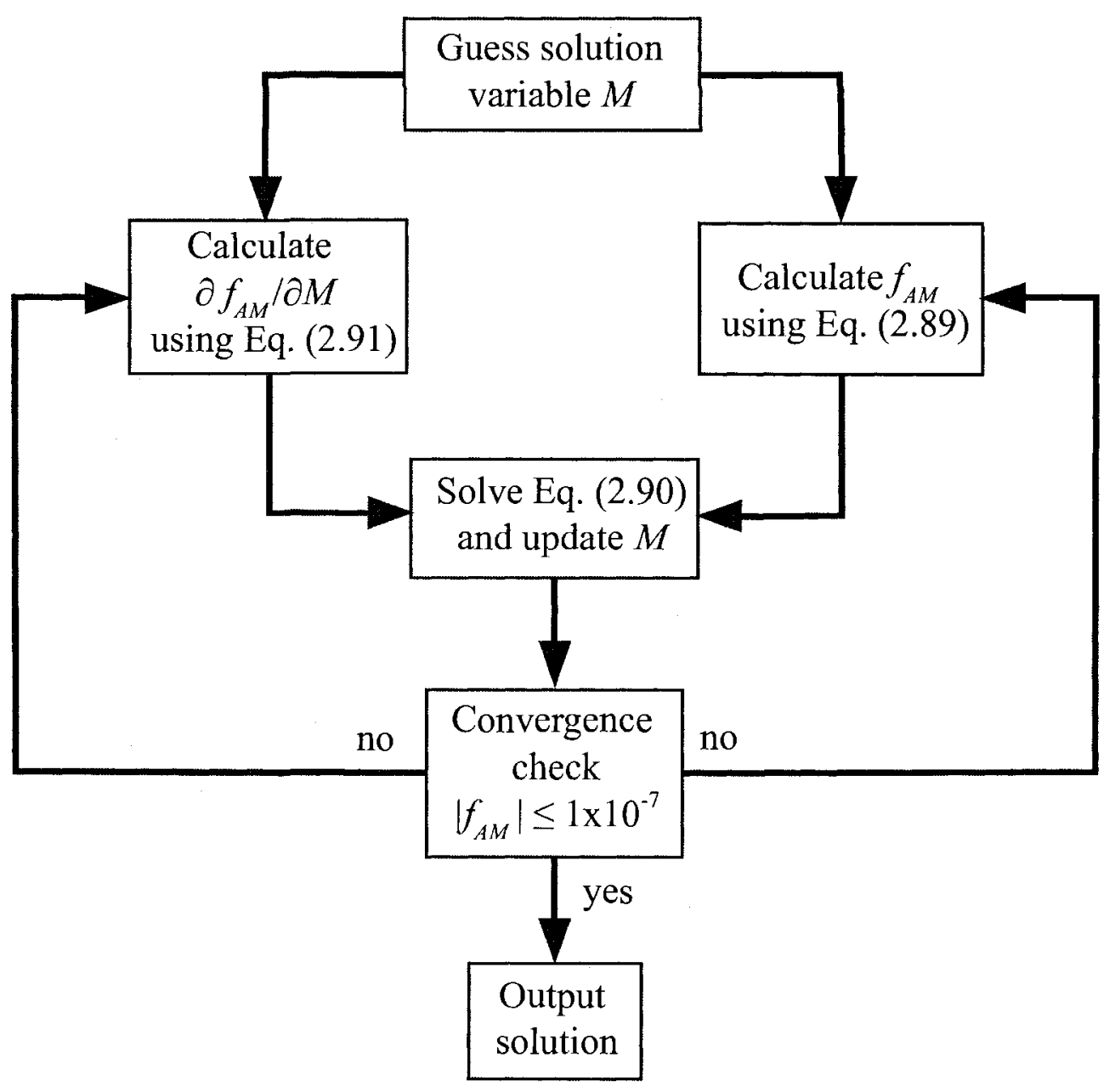

Figure 2.8: Solution of Area-Mach Number Relation 


\section{Chapter 3}

\section{Theory for SMC Ejector}

If one assumes that the pressure distribution used for the unreacting case remains valid under SMC conditions then the equations for the conservation of mass (Eq. (2.4)) and momentum (Eq. (2.16)) remain unchanged. Thus the only thing required is that the energy equation be modified to account for the heat release caused by the secondary combustion inside the ejector. Keeping this in mind one can derive a more general form of the energy equation as,

$$
\sum_{k=1}^{n_{s}} N_{k} \bar{h}_{k}+W_{m} \frac{u_{m}^{2}}{2} \sum_{k=1}^{n_{s}} N_{k}=\dot{m}_{a}\left(h_{a}+\frac{u_{a}^{2}}{2}\right)+\dot{m}_{r}\left(h_{r}+\frac{u_{r}^{2}}{2}\right)
$$

where $n_{s}$ is the number of atomic species being considered. Since chemical reactions are now present in the system, all enthalpy terms must be expressed relative to a reference state. Doing so allows the energy fluxes to be written on a mass-basis as follows:

$$
h+\frac{u^{2}}{2}=\sum_{k=1}^{n_{s}} \frac{Y_{k}}{W_{k}} \bar{h}_{f, k}^{o}+C_{p}\left(T^{o}-T_{r e f}\right)
$$


In addition, the ideal gas law and the speed of sound can be used to simplify the kinetic energy term on the left hand side of $\mathrm{Eq}(3.1)$ to give,

$$
W_{m} \frac{u_{m}^{2}}{2} \sum_{k=1}^{n_{s}} N_{k}=\frac{1}{2} N_{m} \gamma_{m} R_{u} T_{m} M_{m}^{2}
$$

It should be noted at this point, that the kinetic energy term (Eq. (3.3)) does not usually show up in a combustion analysis since the post-combustion velocity is usually small. The present application, however, necessitates that this term be included in the equation since the velocity at the mixed-flow plane of the ejector cannot be considered small. Substituting Eqs. (3.2) \& (3.3) into Eq. (3.1) while invoking the definitions of $\alpha$ and $\theta$ yields the simplified energy equation,

$$
\sum_{k=1}^{n_{s}} N_{k} \bar{h}_{k}+\frac{1}{2} N_{m} \gamma_{m} R_{u} T_{m} M_{m}^{2}=H
$$

where $H$ represents the total energy flowing into the ejector, and is given as,

$$
H=\dot{m}_{r} C_{p, r} T_{r}^{o}\left[(\alpha \theta+1)-\frac{T_{r e f}}{T_{r}^{o}}\left(\alpha\left(\frac{C_{p, a}}{C_{p, r}}\right)+1\right)+\frac{1}{C_{p, r} T_{r}^{o}} \sum_{k=1}^{n_{r}} \frac{Y_{r, k}}{M W_{k}} \bar{h}_{f, k}^{o}\right]
$$

The thermal properties of the reacted mixture can be found on a mass weighted basis, averaging this time over all of the product species to yield,

$$
\begin{array}{r}
C_{p, m}=\sum_{k=1}^{n_{s}} Y_{k} C_{p, k} \\
W_{m}=\sum_{k=1}^{n_{s}} \frac{Y_{k}}{W_{k}} \\
R_{m}=\frac{R_{u}}{W_{m}} \\
\gamma_{m}=\left(1-\frac{R_{m}}{C_{p, m}}\right)^{-1}
\end{array}
$$


Unlike the unreacting case, it is clear at this point that Eq. (3.4) is not sufficient to close the system of equations by itself. The inclusion of chemical reactions has essentially added $n_{s}$ unknowns $\left(N_{k}\right.$ 's) for which additional relationships are required.

\subsection{Gibbs Minimization}

Since equation (3.4) is stated very generally, many different methods can be used. For the present case, the Gibbs Minimization technique [26] will be applied to solve for the chemical composition at the exit plane $(m)$ of the ejector. The full derivation of this method is quite involved, and thus only a brief overview will be presented. Fundamentally, the Gibbs Minimization technique involves minimizing the Gibbs free energy of all the atomic species considered while being constrained by the need for of all the atoms involved in the reactions to be conserved. For this reason it goes beyond the simple assumption of complete combustion, since the species which can form under those conditions, such as unburned hydrocarbons and radicals can be accounted for.

To derive the necessary equations, one first needs to consider the Gibbs free energy of a single species which can be written as,

$$
\bar{g}_{k}=\bar{g}_{f, k}^{o}+R_{u} T_{m}\left[\ln \left(\frac{N_{k}}{N_{m}}\right)+\ln \left(\frac{p_{m}}{p_{r e f}}\right)\right]
$$

while the Gibbs energy of the entire mixture per unit time can be expressed on a molar basis to give,

$$
G=\sum_{k=1}^{n_{s}} N_{k} \bar{g}_{k}=\sum_{k=1}^{n_{s}} N_{k}\left\{\bar{g}_{f, k}^{o}+R_{u} T_{m}\left[\ln \left(\frac{N_{k}}{N_{m}}\right)+\ln \left(\frac{p_{m}}{p_{r e f}}\right)\right]\right\}
$$

One can then differentiate the above expression with respect to $N_{k}$ to obtain the 
following simplified result,

$$
\mathrm{d} G=\sum_{k=1}^{n_{s}} \bar{g}_{k} \mathrm{~d} N_{k}
$$

The conservation of distinct atoms can be expressed in the following form:

$$
\sum_{k=1}^{n_{s}} \eta_{i k} N_{k}=b_{i}, \quad i=1 \rightarrow n_{a}
$$

where $\eta_{i k}$ is the amount of atomic particle $i$ per kmol of species $k$ while $b_{i}$ is the total inflow rate of atomic particle $i$. If one further assumes the usual composition for the entrained air $\left(21 \% \mathrm{O}_{2} 79 \% \mathrm{~N}_{2}\right)$, then $b_{i}$ can be expressed as,

$$
b_{i}=\dot{m}_{r}\left[\sum_{k=1}^{n_{r}} \frac{\eta_{i k} Y_{r, k}}{W_{k}}+\frac{\alpha}{W_{a}}\left(0.79 \eta_{i, N_{2}}+0.21 \eta_{i, O_{2}}\right)\right], \quad i=1 \rightarrow n_{a}
$$

where $n_{r}$ is the number of species present in the rocket plume. At this point one can minimize Eq. (3.11) subject to the constraint of atom conservation (Eq. (3.13)). The method of Lagrange Multipliers is a perfect candidate for this task since it can be used to maximize/minimize a function $f(x, y)$ subject to a constraint function $q(x, y)$. For multiple constraints, the method generalizes to,

$$
\nabla f\left(x_{o}, y_{o}\right)=\sum_{k} \lambda_{k} \nabla q_{k}\left(x_{o}, y_{o}\right)
$$

where $\lambda_{k}$ is a Lagrange multiplier. Taking the derivative of Eq. (3.13) with respect to $N_{k}$ and substituting it along with Eq. (3.12) into the Lagrange formula gives,

$$
\bar{g}_{k}-\sum_{i=1}^{n_{a}} \lambda_{i} \eta_{i k}=0, \quad k=1 \rightarrow n_{s}
$$

where $n_{a}$ is the number of distinct atomic species. It is also convenient at this point 
to non-dimensionalize the above equation by dividing it by $R_{u} T_{m}$ to give,

$$
\widetilde{g}_{k}-\sum_{i=1}^{n_{a}} \widetilde{\lambda}_{i} \eta_{i k}=0, \quad k=1 \rightarrow n_{s}
$$

where

$$
\widetilde{g}_{k}=\frac{\bar{g}_{k}}{R_{u} T_{m}} \quad \widetilde{\lambda}_{i}=\frac{\lambda_{i}}{R_{u} T_{m}}
$$

Solution of the ejector under SMC conditions can be done in a manner similar to the unreacting case except that Eq. (2.23) has now been replaced with Eqs. (3.4), (3.13) and (3.17). Unlike the unreacting case, however, there are now additional $\left(n_{a}+n_{s}\right)$ unknowns which are required to solve for the equilibrium composition.

\subsection{Numerical Solution}

To solve for the post-combustion ejector composition in an efficient manner requires a slightly unintuitive approach since the solution depends on the number of product species. For example, if one tried to solve the system using the Newton-Raphson method with the unknowns being the molar flow rates of the product species $\left(N_{k}\right.$ 's $)$, one would be required to derive and evaluate an $n_{s} \times n_{s}$ Jacobian, which would quickly become cumbersome and inefficient for large numbers of species. In addition, one would also loose some flexibility since the numerical scheme would need major modifications for different numbers of product species. In the present case, the Newton-Raphson method can still be applied, but in a slightly different manner. The solution methodology that will be shown is adapted from reference [26]. The starting point for this method is to apply the Newton-Raphson linearization to a generic functional, $f_{s}$ while using the natural logarithm of $N_{m}, T_{m}$ and the $N_{k}$ 's as the solution 
variables,

$$
\sum_{k=1}^{n_{s}}\left[\frac{\partial f_{s}}{\partial \ln \left(N_{k}\right)}\right]+\frac{\partial f_{s}}{\partial \ln \left(N_{m}\right)} \Delta \ln \left(N_{m}\right)+\frac{\partial f_{s}}{\partial \ln \left(T_{m}\right)} \Delta \ln \left(T_{m}\right)=-f_{s}
$$

Using the following mathematical definition

$$
\frac{\partial y}{\partial \ln x}=x \frac{\partial y}{\partial x}
$$

along with Eq. (3.17) as the $f_{s}$ in equation (3.19), one obtains,

$$
\Delta \ln \left(N_{k}\right)=\Delta \ln \left(N_{m}\right)+\widetilde{h}_{k} \Delta \ln \left(T_{m}\right)-\widetilde{g}_{k}+\sum_{i=1}^{n_{a}} \widetilde{\lambda}_{i} \eta_{i k}, \quad k=1 \rightarrow n_{s}
$$

with

$$
\widetilde{h}_{k}=\frac{\bar{h}_{k}}{R_{u} T_{m}}
$$

In a similar manner, one can also substitute the atomic constraint equation (Eq. (3.13)) into Eq. (3.19) to give,

$$
\sum_{k=1}^{n_{s}}\left[N_{k} \eta_{i k} \Delta \ln \left(N_{k}\right)\right]=-\sum_{k=1}^{n_{s}} \eta_{i k} N_{k}+b_{i}, \quad i=1 \rightarrow n_{a}
$$

At this point the $\Delta \ln N_{k}$ term can be eliminated from equation (3.20) by using Eq. (3.22), resulting in the following expression:

$$
\begin{array}{r}
\sum_{j=1}^{n_{a}}\left\{\sum_{k=1}^{n_{s}} \eta_{i k} N_{k} \eta_{j k}\right\} \widetilde{\lambda}_{j}+\left\{\sum_{k=1}^{n_{s}} \eta_{i k} N_{k}\right\} \Delta \ln \left(N_{m}\right), \quad i=1 \rightarrow n_{a} \\
+\left\{\sum_{k=1}^{n_{s}} \eta_{i k} N_{k} \widetilde{h}_{k}\right\} \Delta \ln \left(T_{m}\right)=b_{i}-\sum_{k=1}^{n_{s}} \eta_{i k} N_{k}+\sum_{k=1}^{n_{s}} \eta_{i k} N_{k} \widetilde{g}_{k}
\end{array}
$$

Equation (3.23) is an important result since it effectively eliminates the $N_{k}$ 's from the numerical procedure and thus significantly improves the computational efficiency 
of the method.

For the present solution it is convenient to consider the total molar flow rate, $N_{m}$, as an independent variable for which one can write,

$$
\sum_{k=1}^{n_{s}} N_{k}-N_{m}=0
$$

The above equation is then used as the next functional $\left(f_{s}\right)$ while applying the same procedure as above to give,

$$
\begin{aligned}
& \sum_{i=1}^{n_{a}}\left\{\sum_{k=1}^{n_{s}} \eta_{i k} N_{k}\right\} \widetilde{\lambda}_{i}+\left\{\sum_{k=1}^{n_{s}} N_{k}-N_{m}\right\} \Delta \ln \left(N_{m}\right) \\
& +\left\{\sum_{k=1}^{n_{s}} N_{k} \widetilde{h}_{k}\right\} \Delta \ln \left(T_{m}\right)=N_{m}+\sum_{k=1}^{n_{s}} N_{k}\left(\widetilde{g}_{k}-1\right)
\end{aligned}
$$

Using the energy equation (Eq. (3.4)) as the last functional, one obtains the final equation required to solve the system as,

$$
\begin{array}{r}
\sum_{i=1}^{n_{a}}\left\{\sum_{k=1}^{n_{s}} \eta_{i k} N_{k} \widetilde{h}_{k}\right\} \widetilde{\lambda}_{i}+\left\{\sum_{k=1}^{n_{s}} N_{k} \widetilde{h}_{k}+E_{K}\right\} \Delta \ln \left(N_{m}\right) \\
+\left\{\sum_{k=1}^{n_{s}} N_{k}\left[\frac{\bar{C}_{p, k}}{R_{u}}+\widetilde{h}_{k}^{2}\right]+E_{K}\right\} \Delta \ln \left(T_{m}\right) \\
=\widetilde{H}-E_{K}+\sum_{k=1}^{n_{s}} N_{k} \widetilde{h}_{k}\left(\widetilde{g}_{k}-1\right)
\end{array}
$$

where

$$
\begin{array}{r}
E_{K}=\frac{1}{2} N_{m} \gamma_{m} M_{m}^{2} \\
\widetilde{H}=\frac{H}{R_{u} T_{m}}
\end{array}
$$

Equations (3.23), (3.25) and (3.26) can be expressed in the familiar matrix form 
$\mathbf{G x}=\mathbf{R}$, with the system matrices defined as,

$$
\mathbf{G}=\sum_{k=1}^{n_{s}}\left[\begin{array}{ccccc}
\eta_{1 k} \eta_{1 k} N_{k} & \cdots & \eta_{1 k} \eta_{n_{a} k} N_{k} & \eta_{1 k} N_{k} & \eta_{1 k} N_{k} \widetilde{h}_{k} \\
\vdots & \ddots & \vdots & \vdots & \vdots \\
\eta_{n_{a} k} \eta_{1 k} N_{k} & \cdots & \eta_{n_{a} k} \eta_{n_{a} k} N_{k} & \eta_{n_{a} k} N_{k} & \eta_{n_{a} k} N_{k} \widetilde{h}_{k} \\
\eta_{1 k} N_{k} & \cdots & \eta_{n_{a} k} N_{k} & N_{k}-N_{m} & N_{k} \widetilde{h}_{k} \\
\eta_{1 k} N_{k} \widetilde{h}_{k} & \cdots & \eta_{n_{a} k} N_{k} \widetilde{h}_{k} & N_{k} \widetilde{h}_{k}+E_{K} & N_{k}\left[\frac{\bar{C}_{p, k}}{R_{u}}+\widetilde{h}_{k}^{2}\right]+E_{K}
\end{array}\right]
$$

and

$$
\mathbf{x}=\left[\begin{array}{c}
\widetilde{\lambda}_{1} \\
\vdots \\
\widetilde{\lambda}_{n_{a}} \\
\Delta \ln \left(N_{m}\right) \\
\Delta \ln \left(T_{m}\right)
\end{array}\right] \mathbf{R}=\left[\begin{array}{c}
b_{1}+\sum_{k=1}^{n_{s}} \eta_{1 k} N_{k}\left(\widetilde{g}_{k}-1\right) \\
\vdots \\
b_{n_{a}}+\sum_{k=1}^{n_{s}} \eta_{n_{a} k} N_{k}\left(\widetilde{h}_{k}-1\right) \\
N_{m}+\sum_{k=1}^{n_{s}} N_{k}\left(\widetilde{g}_{k}-1\right) \\
\widetilde{H}+\sum_{k=1}^{n_{s}} N_{k} \widetilde{h}_{k}\left(\widetilde{g}_{k}-1\right)-E_{K}
\end{array}\right]
$$

The result of using Eq. (3.23) can now be seen very clearly as the system matrix has been scaled down to $\left(n_{a}+2\right) \times\left(n_{a}+2\right)$. Accordingly, the size of the matrix no longer depends on the number of product species but instead on the number of distinct atoms $\left(n_{a}\right)$ involved in the reactions. Since most combustion reactions can be adequately described by various interactions of four atoms $(\mathrm{C}, \mathrm{O}, \mathrm{H}, \mathrm{N})$, the system matrix size would thus be fixed to $6 \times 6$, independent of the number of product species considered. The entire solution process for the reacting ejector is shown in Figure 3.1. While the steps for solving the mass and momentum (Eqs. (2.4) \& (2.16)) conservation 
equations remain the same, solving for the composition involves the inversion of the G matrix starting from an initial guess at the equilibrium composition $\left(N_{m}, N_{k}\right.$ 's $)$ and the post-combustion temperature $\left(T_{m}\right)$. The initial guesses used for the present case are,

$$
T_{m}=2500 \mathrm{~K}, \quad N_{k}=0.1 / n_{s}, \quad k=1 \rightarrow n_{s}
$$

The updated values for the solution variables $\left(\widetilde{\lambda}_{k}, N_{m}, T_{m}\right)$ are then used to solve for all of the $N_{k}$ 's by applying Eq. (3.20) in a sequential manner. Once a solution for the composition and $T_{m}$ is obtained the rest of the ejector solution process continues in the same manner as for the unreacting case (Fig. 3.1). The entire ejector solution under SMC conditions is considered converged when the following conditions are met:

$$
\left|f_{1}, f_{2}\right| \leq 1 \times 10^{-7}, \quad \sum_{k=1}^{n_{s}} N_{k}-N_{m} \leq 1 \times 10^{-7}
$$

In most cases, a converged solution can be obtained in approximately 20 iterations.

To ensure that the numerical procedure remains stable, a damping function is applied to the equilibrium solution at each iteration. For the major species whose $\Delta \ln \left(N_{k}\right)$ is positive, one can calculate the damping factor, $\Upsilon_{1}$, as,

$$
\Upsilon_{1}=\frac{2}{\max \left[\left|\Delta \ln \left(T_{m}\right)\right|,\left|\Delta \ln \left(N_{m}\right)\right|,\left|\Delta \ln N_{k}\right|\right]}
$$

While for the minor species $\left(Y_{k} \leq 1 \times 10^{-8}[26]\right)$ with positive $\Delta \ln \left(N_{k}\right)$ 's one can calculate another damping factor,

$$
\Upsilon_{2}=\frac{\ln \left(1 \times 10^{-4}\right)-\ln \left(N_{k}\right)+\ln \left(N_{m}\right)}{\Delta \ln \left(N_{k}\right)-\Delta \ln \left(N_{m}\right)}
$$


From $\Upsilon_{1}$ and $\Upsilon_{2}$ one can then calculate the overall damping factor,

$$
\Upsilon=\min \left[1, \Upsilon_{1}, \Upsilon_{2}\right]
$$

The damping factor can then be used to update the solution variables,

$$
\begin{array}{r}
{\left[\ln \left(T_{m}\right)\right]^{k+1}=\left[\ln \left(T_{m}\right)\right]^{k}+\Upsilon\left[\Delta \ln \left(T_{m}\right)\right]^{k}} \\
{\left[\ln \left(N_{m}\right)\right]^{k+1}=\left[\ln \left(N_{m}\right)\right]^{k}+\Upsilon\left[\Delta \ln \left(N_{m}\right)\right]^{k}} \\
{\left[\ln \left(N_{k}\right)\right]^{k+1}=\left[\ln \left(N_{k}\right)\right]^{k}+\Upsilon\left[\Delta \ln \left(N_{k}\right)\right]^{k} \quad k=1 \rightarrow n_{s}}
\end{array}
$$




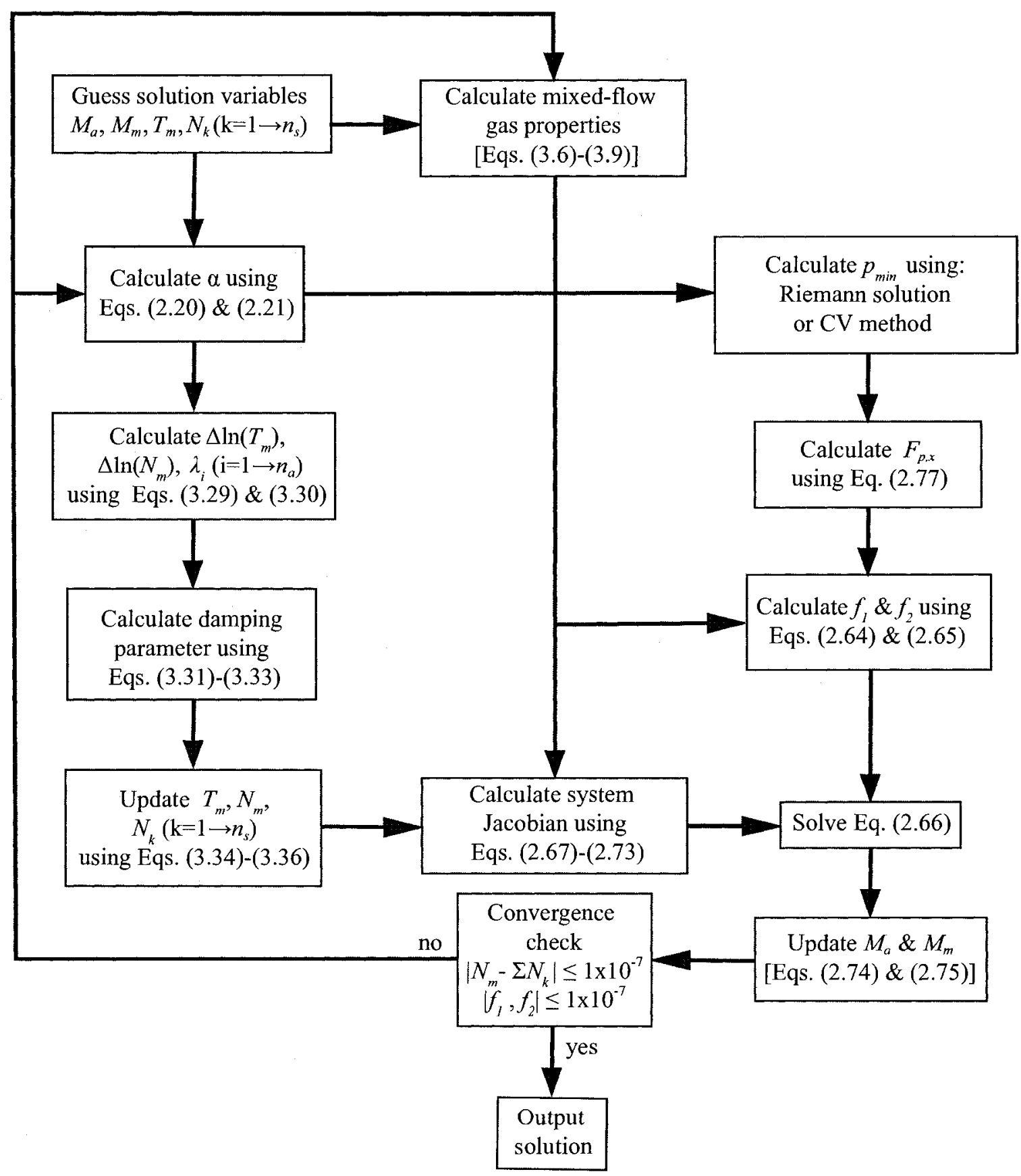

Figure 3.1: Ejector Solution Process (Reacting) 


\section{Chapter 4}

\section{Partially Mixed Ejector Theory}

The theory for the partially mixed ejector is essentially an extension of the fully-mixed theory presented earlier. The scope of the problem is quite broad since all of the flow variables at a given plane will not be uniform for a flow that is not fully mixed. To simplify the analysis, the variation of $T, \rho, p$ and $R$ in the radial direction will therefore be neglected. The only variable that will be allowed to vary is the velocity $u$. With this notion, the conservation of mass is applied to the ejector control volume shown in Fig. 2.1 to yield,

$$
\dot{m}_{r}+\dot{m}_{a}=2 \pi \int_{0}^{r_{m}} \rho u r \mathrm{~d} r
$$

Since the density at the mixed flow plane was assumed to be uniform, it can be taken out of the integral. One can then simplify the equation by using the definition of $\alpha$ to give,

$$
\frac{\dot{m}_{r}}{A_{m}}(\alpha+1)=\rho_{m} \bar{u}_{m}
$$

where $\bar{u}_{m}$ is the area-averaged velocity, defined as,

$$
\bar{u}_{m}=\frac{2}{r_{m}^{2}} \int_{0}^{r_{m}} u r \mathrm{~d} r
$$


Before the mass conservation equation can be simplified further, one needs to derive the energy equation, which can be written as follows:

$$
\dot{m}_{a} C_{p, a} T_{a}^{o}+\dot{m}_{r} C_{p, r} T_{r}^{o}=2 \pi \int_{0}^{r_{m}} \rho u\left(C_{p, m} T+u^{2} / 2\right) r \mathrm{~d} r
$$

The left hand side of the above equation can be simplified in the same manner as for the fully mixed case, by invoking the definitions of $\alpha$ and $\theta$. Furthermore, the integral on the right hand side can be split since the temperature does not vary with $r$. The result can then be divided by $A_{m}$ to give,

$$
\dot{m}_{r} C_{p, r} T_{r}^{o}(\alpha \theta+1)=\rho_{m} A_{m}[C_{p, m} T_{m} \underbrace{\frac{2}{r_{m}^{2}} \int_{0}^{r_{m}} u r \mathrm{~d} r}_{\bar{u}_{m}}+\frac{1}{r_{m}^{2}} \int_{0}^{r_{m}} u^{3} r \mathrm{~d} r]
$$

If one then multiplies and divides the equation by $\bar{u}_{m}^{3}$, one obtains,

$$
\dot{m}_{r} C_{p, r} T_{r}^{o}(\alpha \theta+1)=\rho_{m} A_{m} \bar{u}_{m} C_{p, m}\left[T_{m}+\beta_{e} \frac{\bar{u}_{m}^{3}}{C_{p, m}}\right]
$$

where $\beta_{e}$ is the energy mixing factor, given as,

$$
\beta_{e}=\frac{1}{\bar{u}_{m}^{3} r_{m}^{2}} \int_{0}^{r_{m}} u^{3} r \mathrm{~d} r
$$

At this point it is convenient to define the area-averaged total temperature,

$$
\bar{T}_{m}^{o}=T_{m}+\beta_{e} \frac{\bar{u}_{m}^{2}}{C_{p, m}}
$$

If one further defines the area-averaged Mach number as,

$$
\bar{M}_{m}=\frac{\bar{u}_{m}}{\gamma_{m} R_{m} T_{m}}
$$


then Eq. (4.6) can be written in the following form:

$$
\bar{T}_{m}^{o}=T_{m}\left(1+\left(\gamma_{m}-1\right) \beta_{e} \bar{M}_{m}^{2}\right)
$$

Equations (4.2) and (4.8) can now be substituted into the energy equation, to give the final result,

$$
\bar{T}_{m}^{o}=\frac{C_{p, r}}{C_{p, m}} \frac{(\alpha \theta+1)}{(\alpha+1)} T_{r}^{o}
$$

To finish deriving the mass conservation equation, one needs to restate it in terms of $\bar{M}_{m}$ by multiplying and dividing Eq. (4.10) by the speed of sound and using the ideal gas law to give,

$$
\frac{\dot{m}_{r}}{A_{m}}(\alpha+1)=p_{m} \sqrt{\frac{\gamma_{m}}{R_{m} T_{m}}} \bar{M}_{m}
$$

One can then substitute Eq. (4.8) into the above expression to obtain the final result,

$$
\frac{\dot{m}_{r}}{p_{m} A_{m}} \sqrt{\frac{R_{m} \bar{T}_{m}^{o}}{\gamma_{m}}}(\alpha+1)=\bar{M}_{m}\left(1+\left(\gamma_{m}-1\right) \beta_{e} \bar{M}_{m}^{2}\right)^{1 / 2}
$$

To complete the equation set, one can now apply the momentum equation, which can be written as,

$$
\left(\dot{m}_{a} u_{a}+p_{a} A_{a}\right)+\left(\dot{m}_{r} u_{r}+p_{r} A_{r}\right)-p_{m} A_{m}-\iint_{0}^{L} p_{w}(x) \cdot \mathrm{d} \vec{S}_{x}=\left[2 \pi \int_{0}^{r_{m}} \rho u^{2} r \mathrm{~d} r\right]
$$

The left hand side of the equation above can be simplified in the same manner as Eq. (2.6) while using the ideal gas law on the right hand side to give the following expression:

$$
\frac{\dot{m}_{r} a_{r}^{*}}{p_{m} A_{m}}\left(\alpha \sqrt{\theta} \Gamma \chi_{a}+\chi_{r}\right)-F_{p, x}-1=\frac{1}{R_{m} T_{m}}\left[\frac{2}{r_{m}^{2}} \int_{0}^{r_{m}} u^{2} r \mathrm{~d} r\right]
$$


The above result can then be divided and multiplied by $\bar{u}_{m}^{2}$ while using Eq. (4.7) to get,

$$
\bar{M}_{m}^{2}=\frac{1}{\gamma_{m} \beta_{m}}\left[\frac{\dot{m}_{r} a_{r}^{*}}{p_{m} A_{m}}\left(\alpha \sqrt{\theta} \Gamma \chi_{a}+\chi_{r}\right)-F_{p, x}-1\right]
$$

where $\beta_{m}$ is the momentum mixing factor, defined as,

$$
\beta_{m}=\frac{2}{\bar{u}_{m}^{2} r_{m}^{2}} \int_{0}^{r_{m}} u^{2} r \mathrm{~d} r
$$

Finally, Eqs. (2.24)-(2.27) will be used to solve for the gas properties at the mixedflow plane of the ejector.

To summarize, the foregoing analysis has yielded three equations which govern the partially mixed ejector. Upon inspection of Eqs. (4.9), (4.10) \& (4.12) it is easy to see that they are nearly identical in form to the equations for the fully-mixed case ((2.4), (2.16) \& (2.23)). Since the major departure from the fully-mixed theory was the treatment of the mixed-flow plane $\left(M_{m} \rightarrow \bar{M}_{m}\right.$, etc), all previously defined equations for the $\mu$ and $\chi$ functionals (Eqs. (2.3) (2.10)) apply in this case as well. Consequently, if one can obtain expressions for the two mixing factors, $\beta_{m}$ and $\beta_{e}$ then Eqs. (4.9), (4.10) \& (4.12) can be solved in exactly the same manner as the equations for the fully mixed ejector.

\subsection{Mixed-Flow Velocity Profile}

To properly quantify the two mixing factors, one needs to assume an appropriate velocity profile at the mixed-flow plane of the ejector. In the current treatment, the boundary layer at the mixed-flow plane will be neglected since the intent is to only model the mixing. In addition, since the model is specific to the annular rocket configuration, it can be further assumed that the upper boundary of the shear layer will quickly spread out toward the upper wall. Use of this assumption allows one to 
split the velocity profile into two regions, a shear layer region, which extends from the top wall down, as well as a uniform flow region which extends from the centerline up to the lower boundary of the shear layer.

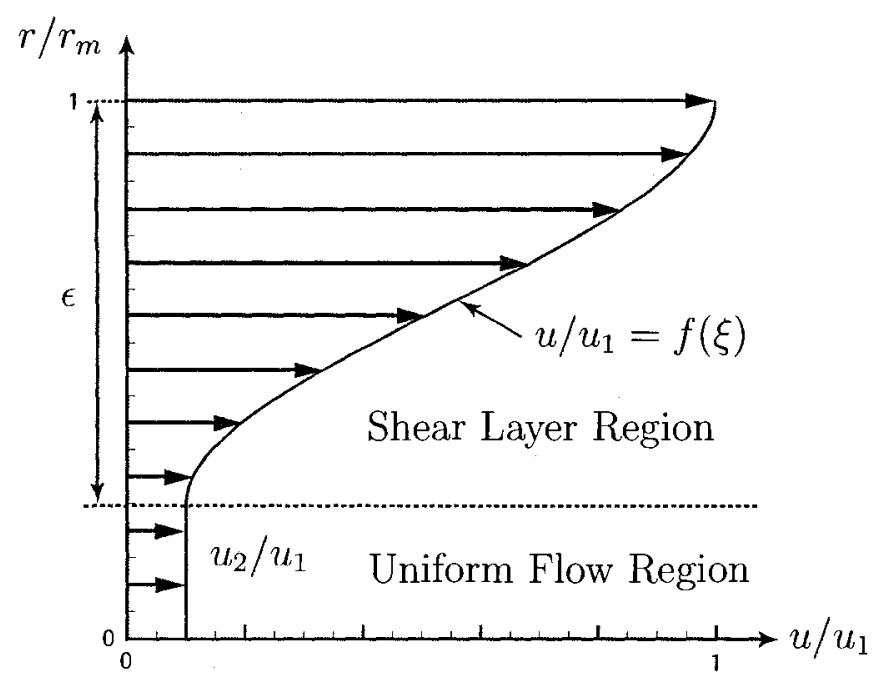

Figure 4.1: Assumed Velocity Profile at Mixed-Flow Plane

A schematic of the composite profile is presented in Figure 4.1, where $\epsilon$ is a relative shear layer thickness defined as,

$$
\epsilon=\frac{\delta_{m}}{r_{m}}
$$

To facilitate the use of specific empirical correlations, it is convenient to express this profile as a function of $u_{2}$ which is the velocity at the lower boundary of the shear layer. One can then make use of the fact that a similarity profile exists for a compressible turbulent shear layer, provided that proper scaling is used for nondimensionalization. The velocity scale is generally taken as the velocity at either one of the shear layer boundaries, while the shear layer thickness, $\delta_{m}$ is used as the length scale. Curve fits for the profile typically involve the well-known Gaussian error function [34]. Although very good agreement between experimental data is obtained when this function is used, it cannot be readily integrated. For this reason, the 
similarity profile will be approximated by using a cubic polynomial of the form:

$$
u(r)=c_{o}+c_{1} r+c_{2} r^{2}+c_{3} r^{3}
$$

If one applies the following boundary conditions:

$$
u\left(r_{m}\right)=u_{1}, \quad \frac{\mathrm{d} u}{\mathrm{~d} r}\left(r_{m}\right)=0, \quad u\left(r_{m}-\delta_{m}\right)=u_{2}, \quad \frac{\mathrm{d} u}{\mathrm{~d} r}\left(r_{m}-\delta_{m}\right)=0
$$

to Eq. (4.15), then the velocity profile can obtained as,

$$
\frac{u}{u_{1}}=u^{*}+\left(1-u^{*}\right)(3-2 \xi) \xi^{2}
$$

where $u^{*}$ is defined as,

$$
u^{*}=\frac{u_{2}}{u_{1}}
$$

and

$$
\xi=\frac{r-\left(r_{m}-\delta_{m}\right)}{\delta_{m}}
$$

Equation (4.16) can then be integrated to yield the expressions required to solve for the integrals appearing in equations (4.3), (4.5) \& (4.13).

$$
\begin{array}{r}
\int_{0}^{r_{m}} u r \mathrm{~d} r=\frac{u_{1} r_{m}^{2}}{2}\left\{(1-\epsilon)^{2} u^{*}+\frac{\epsilon}{10}\left(u^{*}(10-7 \epsilon)+(10-3 \epsilon)\right)\right\} \\
\int_{0}^{r_{m}} u^{2} r \mathrm{~d} r=\frac{u_{1}^{2} r_{m}^{2}}{2}\left\{(1-\epsilon)^{2} u^{* 2}+\frac{\epsilon}{35}\left[2 u^{* 2}(13-10 \epsilon)\right.\right. \\
\left.\left.+9 u^{*}(2-\epsilon)+2(13-3 \epsilon)\right]\right\}
\end{array}
$$




$$
\begin{array}{r}
\int_{0}^{r_{m}} u^{3} r \mathrm{~d} r=\frac{u_{1}^{3} r_{m}^{2}}{4}\left\{(1-\epsilon)^{2} u^{* 3}+\frac{\epsilon}{3080}\left[u^{* 3}(946-763 \epsilon)\right.\right. \\
\left.\left.+u^{* 2}(594-351 \epsilon)+u^{*}(594-243 \epsilon)+(946-183 \epsilon)\right]\right\}
\end{array}
$$

\subsection{Mixing Correlations}

To be able to use Eqs (4.19)-(4.21), additional relations are needed to define $u^{*}$ and $\epsilon$ at the mixed-flow plane of the ejector. Accurately modeling a compressible turbulent shear layer is a difficult task, even with modern computational tools. In addition, the shear layer is also axisymmetric and thus fundamentally different [64] from a simpler, planar case for which most experimental data is available. Since the present model is fairly simple, it lends itself to the use of empirical correlations for plane mixing layers. Although some deviation will be expected, the essential physics of compressible, turbulent mixing will be retained.

The first quantity that is required is the width of the shear layer. One can estimate $\delta_{m}$ by using a correlation for its growth rate. For a planar, compressible, turbulent shear layer, Papamoschou [57] gives the correlation as,

$$
\frac{\mathrm{d} \delta}{\mathrm{d} x}=\frac{0.085(1+\nu)(1-\tau)}{1+\nu \tau} f\left(M_{c}\right)
$$

where

$$
\nu=\frac{\rho_{r}}{\rho_{a}}
$$

and

$$
\tau=\frac{u_{r}}{u_{a}}
$$

The function $f\left(M_{c}\right)$ accounts for the effects of compressibility and is estimated by 
the following empirical relation,

$$
f\left(M_{c}\right)=0.25+0.75 e^{-3 M_{c}^{2}}
$$

where $M_{c}$ is the convective Mach number,

$$
M_{c}=\frac{u_{r}-u_{a}}{a_{r}+a_{a}}
$$

Since a turbulent shear layer grows linearly [64] (Eq. (4.22)), one can estimate the shear layer thickness at the mixed-flow plane using,

$$
\delta_{m} \approx L \frac{\mathrm{d} \delta}{\mathrm{d} x}
$$

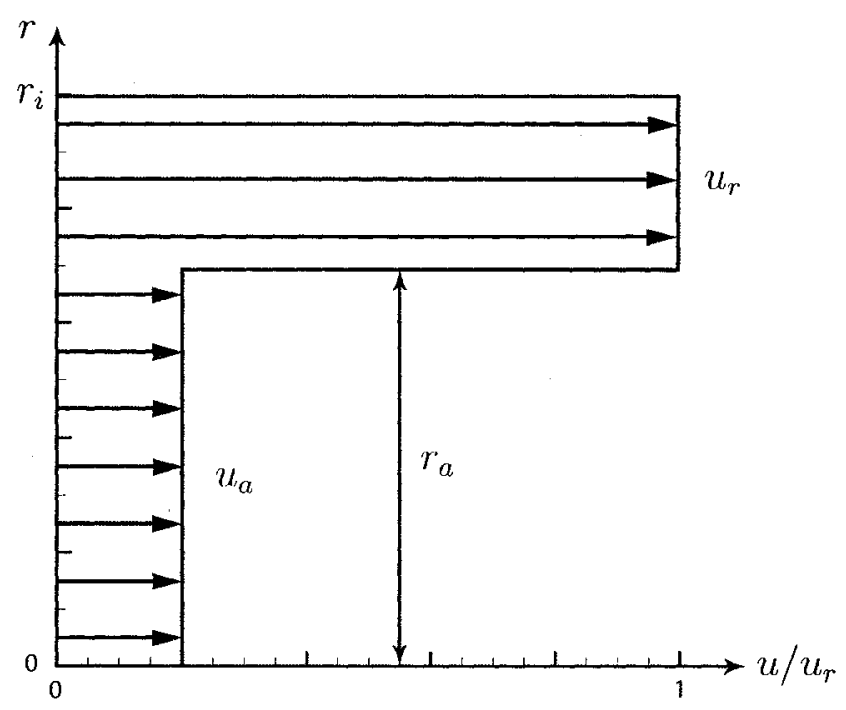

Figure 4.2: Velocity Profile at Ejector Inlet

The last parameter required to quantify the velocity profile is the dimensionless velocity, $u^{*}$. An equation for this quantity can be obtained from Alber and Lees [1], and is written as,

$$
\frac{x}{s_{\theta} \theta_{o}}=\frac{19.7 u^{* 3}}{2-u^{*}-4 u^{* 2}}
$$


where $s_{\theta}$ is a spreading parameter based on the momentum thickness. It is related to the usual turbulent spreading parameter $s$ by the following equation:

$$
\frac{s_{\theta}}{s}=\left[1+\left[\left(\gamma_{m}-1\right) / 2\right] \bar{M}_{m}^{2}\right]^{2}
$$

In the present work the generally accepted value of $s=9.42[1]$ is used. Although Eq. (4.28) can give $u^{*}$ at any $x$-location, only the value at the mixed-flow plane is required for the solution.

To be able to use Eq. (4.28), some way of estimating the initial momentum thickness is required. By definition, the compressible version of the momentum thickness integral can be written as,

$$
\theta_{o}=\int_{0}^{r_{a}}\left(1-\frac{u}{u_{r}}\right) \frac{\rho u}{\rho_{r} u_{r}} \mathrm{~d} r
$$

To simplify the integration, one can assume uniform velocity profiles at the inlet of the ejector, as shown in Figure 4.2. Assuming that the density profiles have the same form, the integral in Eq. (4.30) can be evaluated to yield,

$$
\theta_{o}=\left(\frac{\tau-1}{\nu \tau^{2}}\right) r_{a}
$$

\subsection{Numerical Solution}

As was mentioned earlier, the equations for the partially mixed ejector are nearly identical to those of the fully mixed case. For this reason, the numerical solution of those equations can be carried out in the same manner as shown in Figure 2.6 $\left(T_{m}^{o} \rightarrow \bar{T}_{m}^{o}, M_{m} \rightarrow \bar{M}_{m}\right)$. However, due to the addition of the mixing factors, some modification of the equations as well as the Jacobian is required. Firstly, the mass 
and momentum conservation equations can be restated more conveniently as,

$$
f_{1}=-\gamma_{m} \beta_{m} \bar{M}_{m}^{2}+\left[\frac{\psi \dot{m}_{r} a_{r}^{*}}{\mu_{r} p_{m} A_{m}} \sqrt{\theta} \Gamma\right] \mu_{a} \chi_{a}+\left[\frac{\dot{m}_{r} a_{r}^{*}}{p_{m} A_{m}} \chi_{r}-F_{p, x}-1\right]=0
$$

and

$$
f_{2}=\frac{\dot{m}_{r}}{p_{m} A_{m}} \sqrt{\frac{R_{m} \bar{T}_{m}^{o}}{\gamma_{m}}}\left(\psi \frac{\mu_{a}}{\mu_{r}}+1\right)-\bar{M}_{m}\left(1+\left(\gamma_{m}-1\right) \beta_{e} \bar{M}_{m}^{2}\right)^{1 / 2}=0
$$

Since the partially-mixed formulation altered only the mixed-flow plane as compared to the fully mixed case, all derivatives with respect to $M_{a}$ remain unchanged. One does, however, need to alter the derivatives with respect to the area-averaged Mach number. Differentiating Eqs. (4.32) and (4.33) with respect to $\bar{M}_{m}$ one can write,

$$
\begin{gathered}
\frac{\partial f_{1}}{\partial \bar{M}_{m}}=-2 \gamma_{m} \beta_{m} \bar{M}_{m} \\
\frac{\partial f_{2}}{\partial \bar{M}_{m}}=-\left(1+\left(\gamma_{m}-1\right) \beta_{2} \bar{M}_{m}^{2}\right)^{1 / 2}\left[\frac{\left(\gamma_{m}-1\right) \beta_{e} \bar{M}_{m}^{2}}{2\left(1+\left(\gamma_{m}-1\right) \beta_{e} \bar{M}_{m}^{2}\right)}+1\right]
\end{gathered}
$$

which can be substituted in place of Eqs (2.68) and (2.69) to implement the solution procedure for the partially-mixed model. 


\section{Chapter 5}

\section{Results \& Discussion}

\subsection{Pressure Distribution}

Before using the one-dimensional model to study the performance of a constricted ejector, one must first decide on how to quantify the pressure integral term since the pressure distribution is not known a priori. Two analytical pressure distributions as well as one obtained directly from the computational results of Etele et al. [27] will be compared based on their ability to compute the compression and compression augmentation of a given ejector configuration. The ejector configuration used for the following analysis is the same as the one used to obtain the computational data, with the pertinent parameters presented in Table 5.1.

Table 5.1: Test Conditions

\begin{tabular}{ccccc}
\hline \hline$M_{r}$ & $T_{r}^{o}$ & $p_{r}^{o}$ & $\gamma_{r}$ & $\sigma$ \\
\hline 3.1 & $2316 \mathrm{~K}$ & $58 \mathrm{~atm}$ & 1.27 & 0.1 \\
\hline \hline$\alpha$ & $T_{a}^{o}$ & $p_{a}^{o}$ & $C_{R}$ & $L / D$ \\
\hline 0.75 & $279 \mathrm{~K}$ & $58.7 \mathrm{kPa}$ & 0.25 & 5 \\
\hline \hline
\end{tabular}


A comparison of the three different pressure distributions for the case of $25 \%$ area constriction is shown in Figure 5.1. The wall pressure distribution from CFD simulations (solid curve) has been included as well and shows a shock train structure that is typical of supersonic internal flows [7]. For comparison purposes, this pressure distribution is integrated numerically and input into the ejector equation solver. For these cases, $F_{p, x}$ is calculated independent of the ejector solution because for fixed inlet conditions ( $p_{a}^{o}$ and $\alpha$ ), the mixed-flow pressure could not be matched to the exit pressure from the CFD results. Matching the pressures would increase the entrainment ratio in the theoretical case and would thereby invalidate the contrast with the CFD data.

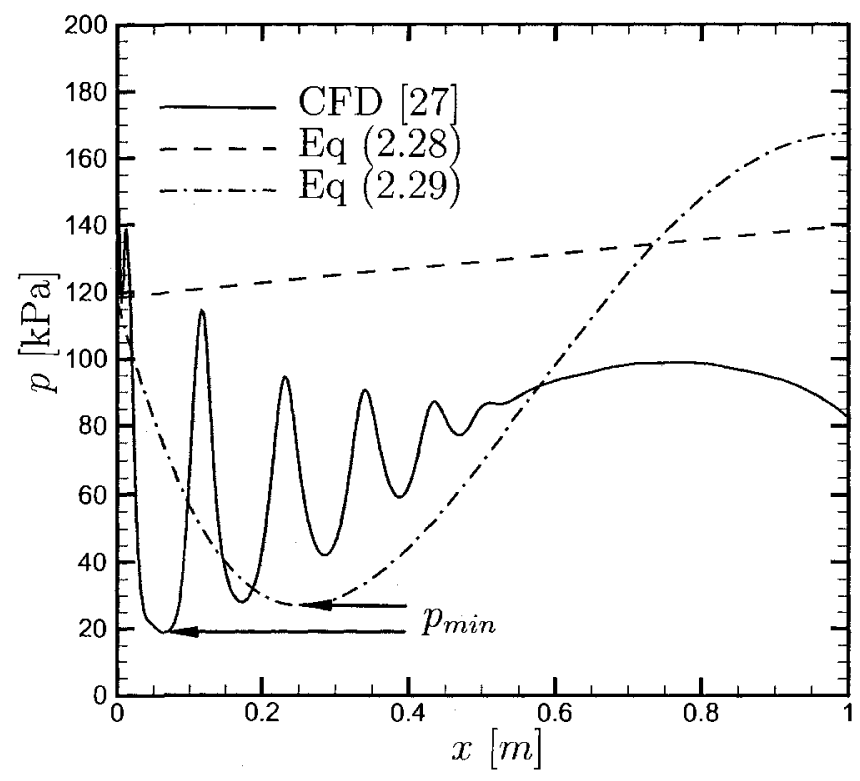

Figure 5.1: Wall Pressure Distributions

If the polynomial pressure distribution is used, a choice must be made as to which method of calculating $p_{\min }$ to adopt for the present study. To assess the relative accuracy of both techniques, the variation of the minimum rocket stream pressure for various inlet conditions is calculated using both the Riemann and CV 
methods. The values of $p_{\min }$ predicted by the Riemann method are independent of grid spacing provided that $\Delta x$ is sufficiently small (a value of $5 \mathrm{~mm}$ is used). Total air pressure $\left(p_{a}^{o}\right)$ is chosen for the ordinate since it captures the variation in both flight altitude and Mach number (at constant $\alpha$ ) without the need for a specific trajectory. Computational data for this case is only available at one flight condition $\left(p_{a}^{o}=58.7\right.$ $\mathrm{kPa}$ ) and is shown in Figure 5.2 along with the theoretical curves.

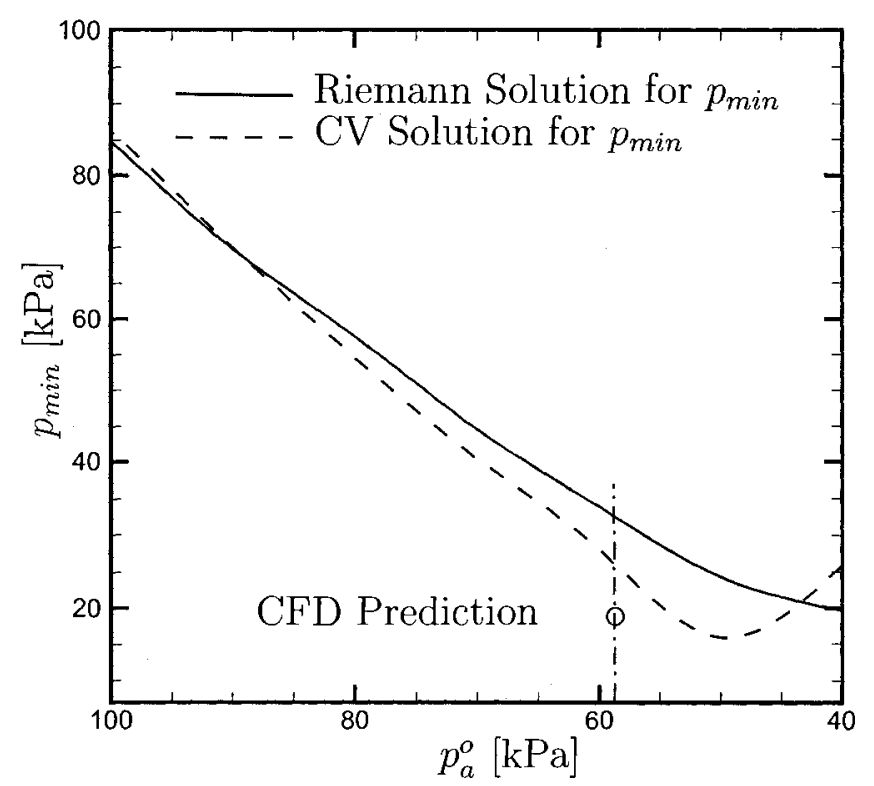

Figure 5.2: Minimum Rocket Stream Pressure at a Fixed Air Mass Flow Rate

Both methods show a similar exponential trend, although the CV method tends to have a steeper slope. The total pressure at which the air stream chokes differs between the two methods as well. Comparing the minimum values in the figure shows that for the given conditions, the Riemann solution chokes at a pressure of $40 \mathrm{kPa}$ while the $\mathrm{CV}$ solution chokes earlier, at $50 \mathrm{kPa}$. The $\mathrm{CV}$ solution curve also shows a minimum at the point where the air stream chokes, after which $p_{\min }$ begins to increase. The minimum occurs because the mass flow rate of the air is held constant to obtain the 
solutions presented in Figure 5.2. Furthermore, since the mass flow rate at sonic conditions is directly proportional to the flow's total pressure and sonic throat area, when $p_{a}^{o}$ is decreased $A_{a}^{*}$ has to increase. When this occurs the area of the rocket stream decreases $\left(A_{r}\right)$, which yields a smaller $M_{r}$ and thus a higher pressure $\left(p_{\min }\right)$. This behaviour makes sense from a physical standpoint, since one would expect the choking of the air stream to place a lower bound on $p_{\min }$.

The Riemann solution, on the other hand, does not show the same type of limiting behaviour, since the choking condition was used to stop the solver before the expansion of the rocket plume was complete. The discrepancy in this case can come from two sources. The first is the Riemann solver itself, which may be over-estimating the extent to which the rocket stream can expand. The other option is that the entrainment ratio, which is governed by Eqs. (2.4), (2.16) \& (2.23) is being overestimated at choked airflow conditions. In this case, the Riemann solver may be more representative of the true flow physics, and the inflow condition being imposed by the governing equations is unrealistic. In either case it is clear that using the Riemann approach with the overall ejector solution procedure is producing anomalies when the air flow chokes, while the CV method seems to be better suited to the present approach.

Overall, both methods are in good agreement with each other due to the common assumptions used by both techniques. From the figure, it is also clear that both methods predict a value of $p_{\min }$ that is $60-70 \%$ higher than the CFD prediction. The reason for the discrepancy is tied to the presence of the shear layer which entrains fluid from both streams and facilitates the transfer of mechanical energy. This causes the total pressure and the mass flow rate of the rocket stream to decrease as the mixing progresses, which means that the flow is not isentropic. These facts are clearly evident in Table 5.2, which shows that both the mass flow rate and the velocity of the rocket stream in the CFD results are lower than the theoretical values. Another reason is the fact that the flow in this expansion region is highly two-dimensional and would 
Chapter 5. Results \& Discussion

thus be able to expand to a lower pressure than an idealized, quasi-one-dimensional flow.

Table 5.2: Comparison of Flow Variables at Point of Maximum Expansion for $p_{a}^{o}=58.7 \mathrm{kPa}$

\begin{tabular}{lccc}
\hline \hline Variable & Riemann & CV & CFD [27] \\
\hline$\dot{m}_{r}[\mathrm{~kg} / \mathrm{s}]$ & 2.5 & 2.5 & 2.2 \\
$u_{r}[\mathrm{~m} / \mathrm{s}]$ & 1955 & 1974 & 1749 \\
$p_{\min }[\mathrm{kPa}]$ & 32.5 & 26.84 & 18.77 \\
\hline \hline
\end{tabular}

Although the minimum rocket pressure differs from CFD predictions, one must keep in mind that it is not the pressure distribution itself but rather $F_{p, x}$ that is of interest. Consequently the actual numerical value of $p_{\min }$, provided it is at least in the right range of values is not particularly important. Given that both methods yield similar results, the choice will be made based on how realistically the physics of the flow are represented. Since the CV method was shown to be the best suited to the overall solution procedure, it will be used to quantify $p_{\min }$ when the polynomial pressure distribution is used.

\section{$5.2 \quad$ Ejector Performance}

With a suitable method chosen for quantifying one of the pressure distributions, the focus can shift to the ejector itself. Since a pressure distribution along the wall needs to be assumed, there is some uncertainty in terms of which one should be used. Theoretical results for the compression and compression augmentation factor using each of the three pressure distributions are compared to the computational results in Figures 5.3 \& 5.4 respectively. Combustion inside the ejector is neglected and the data is obtained for each value of $C_{R}$ by adjusting the mixed-flow pressure until an entrainment ratio of approximately 0.75 is achieved. 


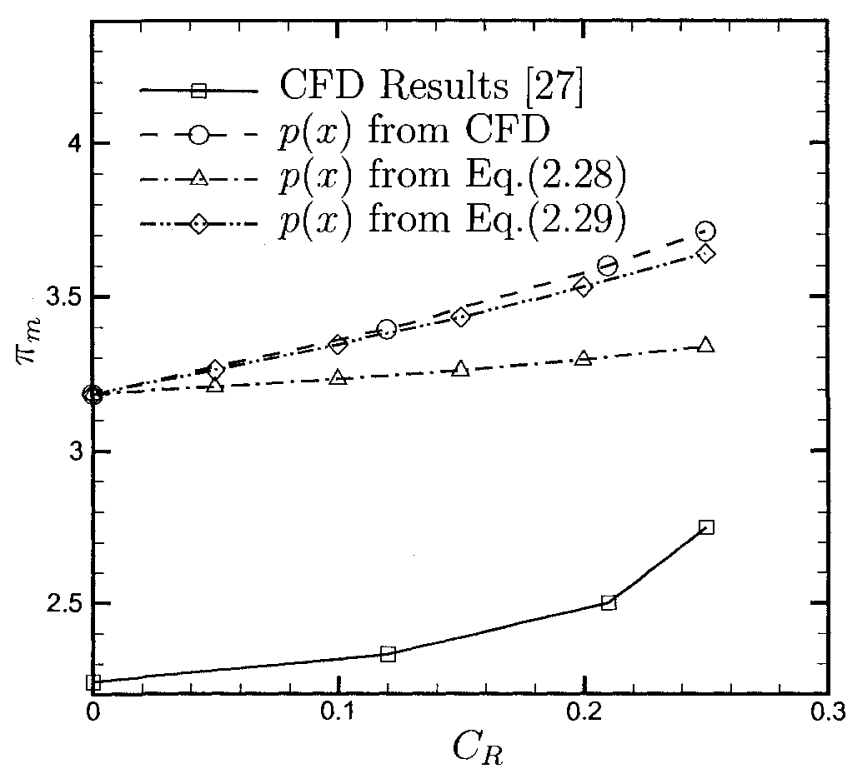

Figure 5.3: Ejector Compression Ratio $\pi_{m}$, theoretical (dashed lines) and CFD (solid line) results

Studying Figure 5.3, it is clear that the analytical solution gives an ejector compression factor that is higher than the CFD prediction by 30-40\% depending on the contraction ratio. This type of discrepancy is not unexpected since the theoretical model is a great simplification of the complex ejector flowfield. The two major effects, which the theory does not consider, is that the flowfield is highly turbulent and may also not be fully mixed. Both of these are essentially losses and would act to decrease the compression factor. In the case of the present CFD results, the configuration considered by Etele et al. [27] was about $93 \%$ mixed based on an outflow mixing parameter (similar to $\beta_{m}$ ) which uses the curvature of the velocity profile to ascertain the extent of mixing (i.e., more uniform velocity profile implies better mixing). The flow was also fully turbulent since the width of the mixing zone encompassed the entire exit plane of the ejector. Since turbulence and mixing are related they can not be readily decoupled into separate effects. Nonetheless, because the flow in the 
computational case is close to being fully mixed, it is clear that turbulence plays a much more important role.

To examine how well the analytical model predicts air entrainment, it is necessary to match the ejector's exit conditions $\left(p_{m}\right)$ so that the inflow conditions can be compared. This type of comparison reveals that the theory also predicts a higher secondary (air) mass flow rate, and thus a higher value of $C_{R}$ at which the entire ejector chokes. For example, when $C_{R}=0.25$, the analytical model predicts an entrainment ratio that is approximately $52 \%$ higher than the CFD results would suggest. This discrepancy is tied to viscous effects as well as the turbulent nature of the flowfield which have a strong influence on the shape (or curvature) of the velocity profile and thus limit the amount of mass that can be passed through a given cross section.

Despite the higher values of $\pi_{m} \& \alpha$ predicted by the analytical model, the trends are well represented, especially when the wall pressure distribution is defined either by Eq. (2.29) or obtained directly form the CFD simulations (top two curves). In fact, in both of these cases, the rate of increase of $\pi_{m}$ with decreasing exit area is well captured as shown in Figures 5.3 \& 5.4. Comparing the top two curves in Figure 5.3, it is evident that both give nearly identical results for $\pi_{m}$, even though the mixed-flow pressures for the two distributions (see Fig. 5.1) are quite different. This is an interesting result, because using the wall pressures extracted from the CFD simulations to quantify $F_{p, x}$ represents the accuracy limit of the theoretical integral analysis. This would seem to suggest that the discrepancy in $\pi_{m}$ is not caused by the pressure distribution, but rather by neglecting the effects mentioned earlier. It also means that within the framework of the theory presented herein, Eq. (2.29) is indeed a reasonable approximation of the integral of the wall pressure $\left(F_{p, x}\right)$.

If one assumes that the viscous and turbulent effects stay nearly constant with $C_{R}$, than they can be effectively eliminated by dividing the compression factors in Fig. 5.3 by $\left[\pi_{m}\right]_{C_{R}=0}$. The result is the compression augmentation factor, $\bar{\pi}_{m}$, which provides 


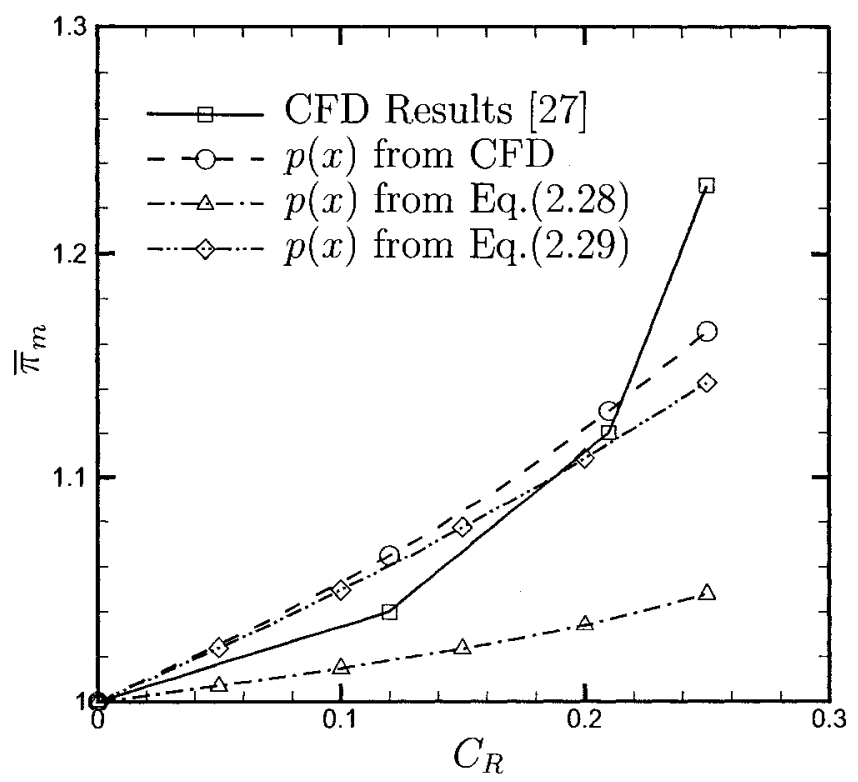

Figure 5.4: Ejector Compression Augmentation $\bar{\pi}_{m}$, theoretical (dashed lines) and CFD (solid line) results

a more convenient basis for comparison. Figure 5.4 shows that the analytical model predicts $\bar{\pi}_{m}$ much better than the compression factor itself. The linear distribution (Eq. (2.28)) predicts a much lower value of $\bar{\pi}_{m}$ and shows a much slower rise with increasing area constriction compared to the computational results. The exponential rise in $\bar{\pi}_{m}$ seems to be reasonably represented by the polynomial up to about $25 \%$ constriction, at which point the two curves begin to diverge. The divergence is due to the discrepancy in the choking point, where for the given conditions $\left(C_{R}=0.25\right.$, $\alpha=0.75)$ the theory predicts a Mach number of only 0.61 at the ejector exit, while the CFD results suggest that the flow is choked on an area-averaged basis. When the wall pressures extracted from the CFD simulations are used, the results are nearly identical to those obtained from using Eq. (2.29), diverging only slightly for higher values of constriction.

Based on the results presented so far it is clear that the polynomial $p(x)$ does an 
adequate job of modeling the ejector's performance within the accuracy limits of the theoretical analysis. More importantly, the exponential trends in both $\pi_{m}$ and $\bar{\pi}_{m}$ are reproduced reasonably well up to the choking limit. Due to the convenience of a closed-form expression, as well as its relative accuracy, Equation (2.29) will be used to quantify $F_{p, x}$ for the remainder of this study. It should be kept in mind, however, that the fully-mixed theory lacks the performance loss mechanisms commonly encountered in ejectors (viscosity, turbulence). Accordingly, the theoretical values of $\pi_{m}$ would be different from a real ejector, and could be on the order of $40 \%$, as is the case for the present comparison.

\subsection{Effects of Combustion}

Although good agreement has been seen between theory and CFD, the results presented so far were obtained at operating conditions which are not representative of an actual ejector. Specifically, the preceding data was obtained at an unrealistically low equivalence ratio to avoid combustion and thus allow a comparison under a mixing only basis. This does not occur in practice since rockets are generally operated at equivalence ratios much higher than 0.2 . To investigate the performance of the ejector in this regime one must now account for the effects of combustion. Since the SMC mode is of particular interest, one must look at the ejector under fuel rich as well as fuel lean conditions. A wide operating range such as this necessitates the need to account for dissociated species as well as products of incomplete combustion. This makes the Gibbs Minimization technique particularly well suited for this task as any number of various product species can be assumed to occur. Use of the equilibrium assumption is warranted for these conditions since it has been validated for the supersonic flowfield of the rocket using a CFD code which accounts for finite-rate combustion kinetics [26]. The product species were chosen by consulting the same 
Kerosene/Oxygen combustion model [26] that was used to compute the chemically reacting flow field of the rocket. For the present computations, a post-combustion mixture composed of 17 species was assumed and is summarized in Table 5.3.

\section{Table 5.3: Assumed Product Species}

\begin{tabular}{cccccc}
\hline \hline 1. $\mathrm{CO}_{2}$ & 2. $\mathrm{CO}$ & 3. $\mathrm{CH}_{3}$ & 4. $\mathrm{CH}_{4}$ & 5. $\mathrm{C}_{2} \mathrm{H}_{2}$ & 6. $\mathrm{C}_{2} \mathrm{H}_{4}$ \\
7. $\mathrm{O}_{2}$ & 8. $\mathrm{O}$ & 9. $\mathrm{OH}$ & 10. $\mathrm{N}_{2}$ & 11. $\mathrm{N}$ & 12. $\mathrm{NO}$ \\
13. $\mathrm{H}_{2} \mathrm{O}$ & $14 . \mathrm{H}_{2}$ & 15. $\mathrm{H}$ & 16. $\mathrm{HO}_{2}$ & 17. $\mathrm{H}_{2} \mathrm{CO}$ & \\
\hline \hline
\end{tabular}

In the following analysis the effect of the rocket equivalence ratio will be quantified. Since $\phi_{r}$ alters the combustion inside the rocket, it affects the combustion inside the ejector indirectly through the inflow conditions (temperature, composition). For this reason, one must be careful to distinguish between the combustion inside the rocket (primary) and the combustion inside the ejector (secondary) when interpreting the results since both are present during SMC operation.

The results for the ejector compression factor, $\pi_{m}$ at various rocket equivalence ratios are presented in Figure 5.5, with the curve for the case when combustion is absent shown as a reference. The points at which the curves terminate represent the contraction ratios at which the ejector chokes. The foreshortening of the curves for $\phi_{r} \geq 1$ relative to the reference case therefore indicates that the flow is being thermally choked. Figure 5.5 shows that the extent of choking at $\phi_{r}=2.5$ is approximately the same as at Stoichiometric conditions, while the $\phi_{r}=2$ configuration chokes at a slightly higher contraction ratio. Overall, the amount of possible area constriction is reduced by no more than $14 \%$. This represents a significant reduction in the possible operating range, and is related to the secondary combustion which increases the energy of the mixed flow. One can quantify this with a combustion temperature rise, $\left(\Delta T_{c}\right)$ which can be calculated by solving the same ejector configuration with the secondary combustion suppressed and taking the difference in mixed 
flow temperatures. The relative combustion temperature rise is plotted in Figure 5.6 for a fixed constriction ratio.

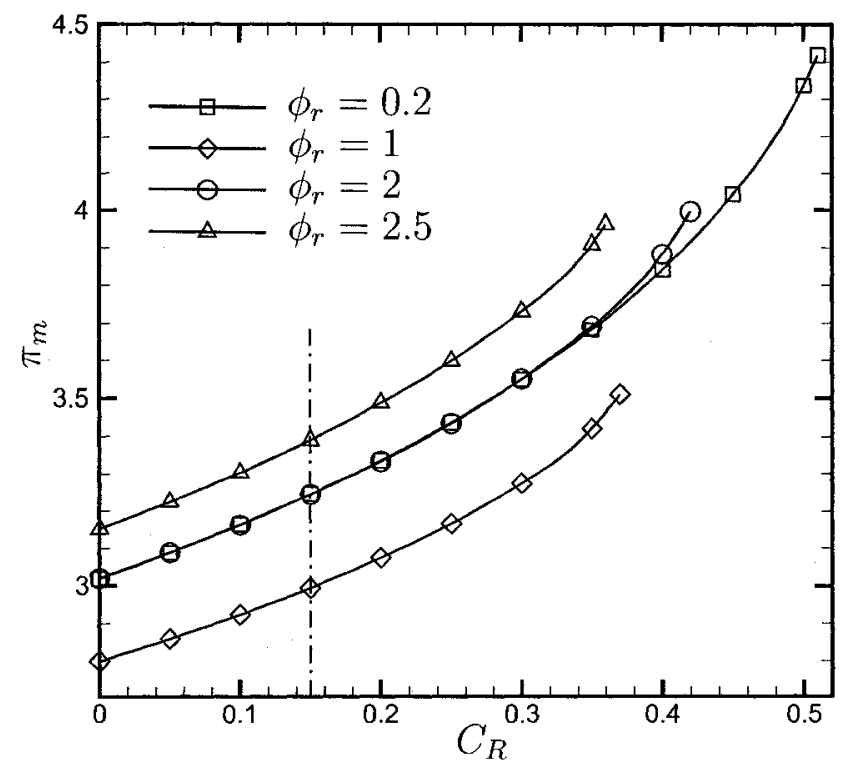

Figure 5.5: Ejector Compression Under SMC Conditions

The equivalence ratios at which the compression data in Figure 5.5 is obtained have been highlighted in Figure 5.6 for clarity. Focusing on these points, it is evident that the relative temperature rise at Stoichiometric conditions is approximately the same as for an equivalence ratio of 2.5. Furthermore, $\Delta T_{c}$ is about $12 \%$ lower at $\phi_{r}=2$ as compared to the other two conditions. If one then compares the choking points of the ejector in Figure 5.5 to the the trends observed in Fig. 5.6 it becomes clear that it is the drop in the heat release at $\phi_{r}=2$ which is responsible for extending the possible constriction ratio range of that configuration.

In addition to thermal choking, secondary combustion also affects the compression factor itself. Shown in Figure 5.7 is the compression augmentation factor which in this case is normalized with $\left[\pi_{m}\right]_{\phi_{r}=0.2}$ at a constriction ratio of $15 \%$. It is evident from the figure that the manner in which $\bar{\pi}_{m}$ is affected, whether detrimental or ben- 


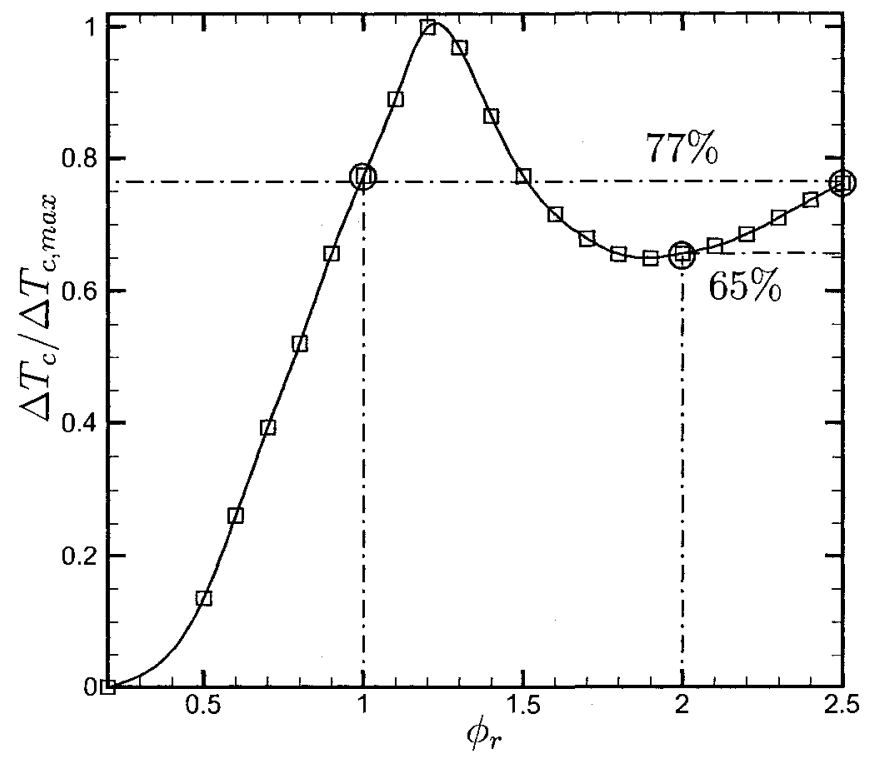

Figure 5.6: Relative Change in Combustion Heat Release $\left(C_{R}=0.15\right)$

eficial, depends largely on the regime in which the rocket is operating in. The lowest performance occurs near Stoichiometric conditions $\left(\phi_{r}=1.2\right)$, with a compression factor that is approximately $8 \%$ lower than the reference case. During fuel rich operation, the trend reverses and compression begins to increase with the equivalence ratio. When the equivalence ratio reaches a value of two, $\bar{\pi}_{m}$ reaches unity, implying that compression has been fully recovered. Further increasing $\phi_{r}$ begins to have a beneficial effect on compression, with a $4 \%$ increase in performance over the reference case.

To get a better grasp on the fundamental reasons behind the behaviour of the compression factor one needs to examine the flow variables at the mixed-flow plane. For this comparison the mixed-flow pressure and Mach number are normalized relative to their respective maximum values and plotted in Figure 5.8. In the fuel-lean regime the pressure and Mach number display opposing trends and reach their respective minimum and maximum values at $\phi_{r}=1.2$. At Stoichiometric conditions the Mach 


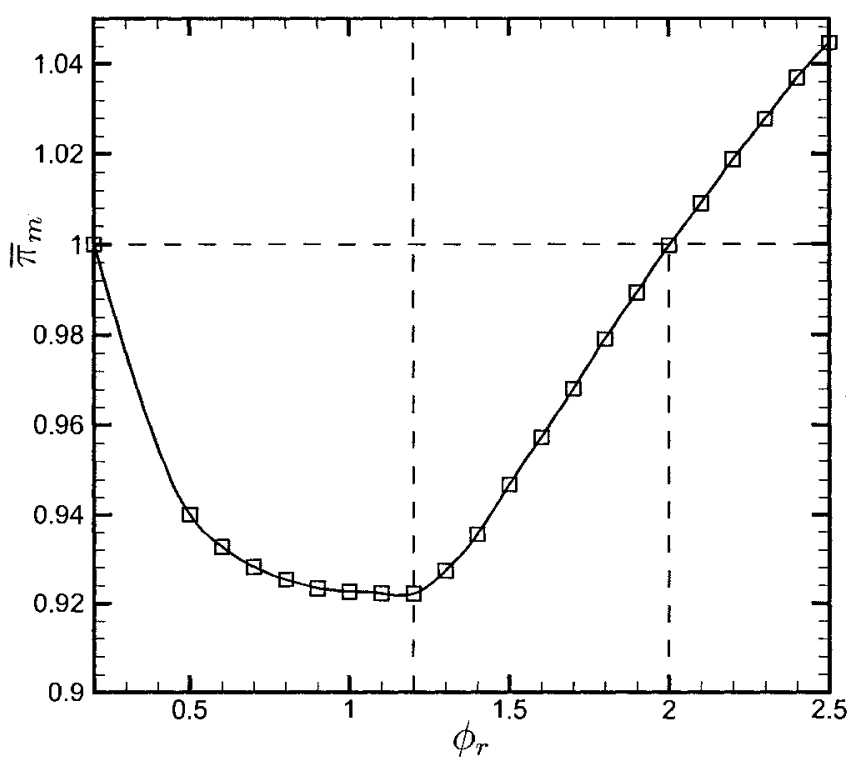

Figure 5.7: Compression Augmentation at SMC Operation $\left(C_{R}=0.15\right)$

number reaches $94 \%$ of its maximum value while the pressure drops to $87 \%$ of its maximum. Although both variables change by approximately the same amount $(\approx$ $13 \%$ ) as compared to the reference case, one must keep in mind that the total pressure is directly proportional to the static pressure. The result of this relationship is that in the fuel-lean regime, the increase in $M_{m}$ is not enough to offset the drop in $p_{m}$, which is why the Stoichiometric case in Fig. 5.5 shows the lowest values of $\pi_{m}$. In the fuelrich region, the pressure begins to increase, and reaches $98 \%$ of its maximum value at $\phi_{r}=2$ and remains unchanged at higher equivalence ratios. At this condition, the Mach number is $9 \%$ higher than at $\phi_{r}=0.2$, which is why the compression ratio at $\phi_{r}=2$ is nearly equal to that of the reference case. When the equivalence ratio increases beyond 2, the Mach number begins to increase once again. Since the pressure at $\phi_{r}=2.5$ is nearly unchanged from the previous condition $\left(\phi_{r}=2\right)$, it is the larger Mach number in this case which causes the compression ratio to exceed the reference value. 


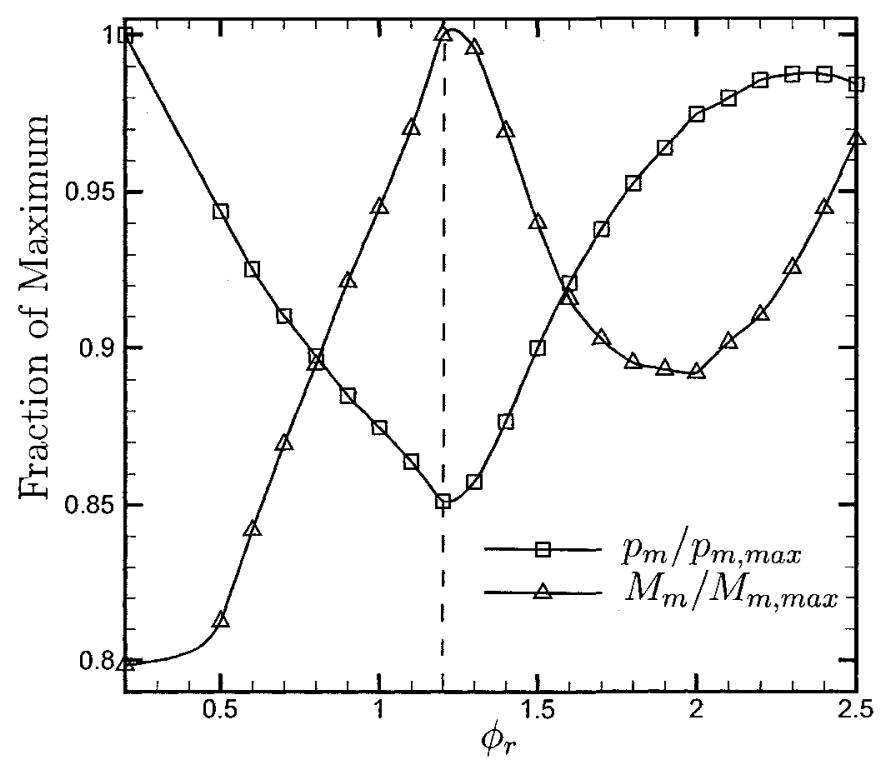

Figure 5.8: Relative Changes in Pressure and Mach Number $\left(C_{R}=0.15\right)$

The results presented so far demonstrate the effects of the rocket equivalence ratio on ejector performance. While changing $\phi_{r}$ affects the combustion inside the ejector, it also influences the combustion inside the rocket. From the point of view of the ejector, changing the equivalence ratio alters the inflow conditions such as the temperature and composition of the rocket stream. This means that even if combustion inside the ejector did not occur, changing $\phi_{r}$ would affect performance by virtue of the changing inlet conditions $\left(T_{r}^{o}, \gamma_{r}\right)$. With the present model one can isolate the effect of combustion inside the rocket (primary combustion) by obtaining a solution under the same conditions while suppressing the secondary combustion inside the ejector. This result can then be compared to the case when both primary \& secondary combustion occur inside the rocket and the ejector respectively. Presented below in Figures 5.9 and 5.10 are the mixed flow temperature and compression factor for each case.

Studying Figure 5.9, very similar trends in the mixed-flow temperature are observed for both cases. In each case, the temperature rises to a maximum value near 


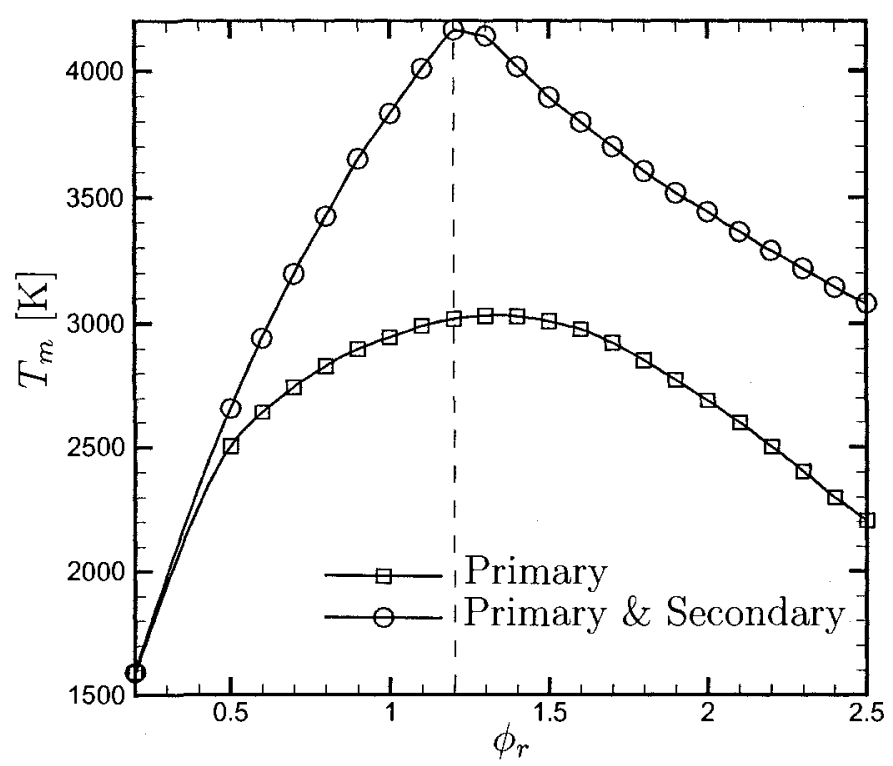

Figure 5.9: Ejector Mixed-Flow Temperature $\left(C_{R}=0.15\right)$

a Stoichiometric equivalence ratio, after which it slowly decreases as $\phi_{r}$ is increased further. When primary and secondary combustion are occurring, the maximum temperature is $38 \%$ higher with a sharper peak, although the equivalence ratio at which the maximum occurs differs by less than $10 \%$ in both instances. Furthermore, during fuel rich operation, the temperatures for both cases decrease at almost the same rate. Given the difference in $T_{m}$ between the two curves, the effects of secondary combustion inside the ejector are therefore important for all values of $\phi_{r}>0.5$. However, from these results it is also clear that the primary combustion has a significant effect on $T_{m}$ since it responsible for about $60 \%$ of the temperature rise.

Moving on to the compression factor, one notes the similarities between Figures $5.9 \& 5.10$ in that the shape of the $\pi_{m}$ curves are very similar. There is however a larger difference in the equivalence ratios at which the minimum values occur. When considering only the primary combustion, the minimum occurs in the fuel-lean region, at $\phi_{r}=0.7$, while the addition of secondary combustion inside the ejector shifts it to 


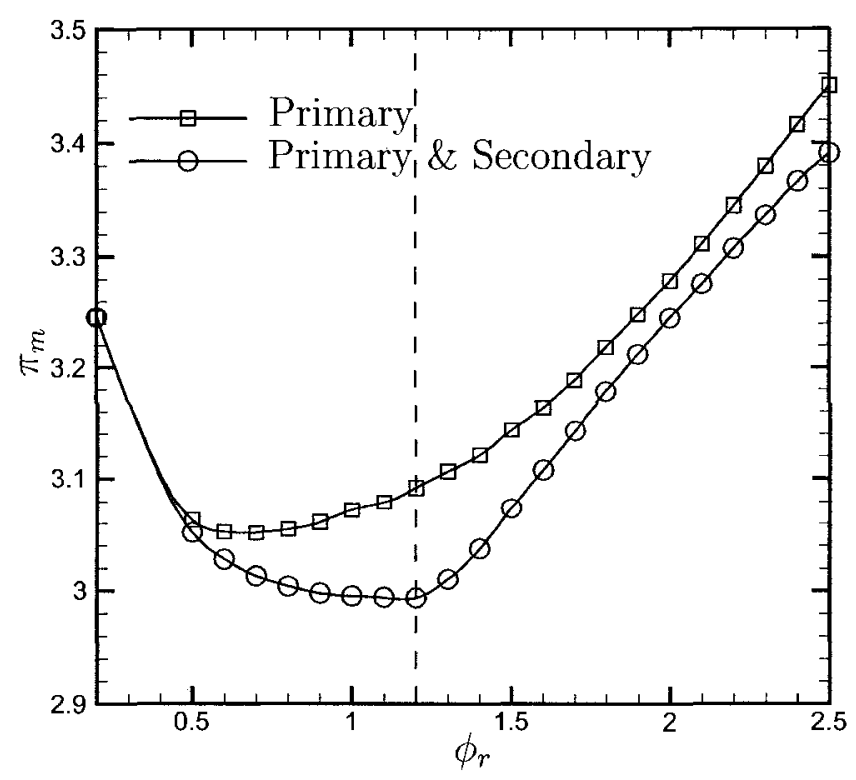

Figure 5.10: Effect of Rocket Temperature on Ejector Compression $\left(C_{R}=\right.$ $0.15)$

a fuel-rich value of 1.2. Unlike Fig. 5.10, secondary combustion seems to have a much weaker effect on the value of $\pi_{m}$ since the shift between the two curves is significantly smaller, especially in the fuel-rich regime. The maximum difference in compression between the two cases is about 3\% at Stoichiometric conditions, and decreases to less than $2 \%$ for higher equivalence ratios. However, this is actually a notable difference since the overall drop in compression at Stoichiometric conditions is only $8 \%$ for all values of $C_{R}$ below choking (see Fig. 5.5). Thus despite the seemingly small difference between the curves for values of $\phi_{r}>0.5$, secondary combustion is in fact significant and should not be neglected.

The secondary combustion that occurs inside the ejector is distinctly different from what happens inside the rocket, since the reactant mixture is now composed of radicals and reacted species. In addition to this, the combustion inside the rocket has consumed all of the Kerosene which means that an entirely different set of reactions 
will be the major drivers of the heat release inside the ejector. To fully understand which reactions are important one needs to examine the product and the reactant composition. For a proper comparison, the rocket composition needs to be calculated relative to the total mass flow of the ejector. For a general species, which can occur in both the rocket and the air stream ( such as $\mathrm{O}_{2}$ ) one can calculate the mass fraction of the $\mathrm{k}^{\text {th }}$ species as,

$$
Y_{k, i}=\frac{Y_{k, r}+\alpha Y_{k, a}}{\alpha+1}
$$

If the species exists only in the rocket stream (such as $\mathrm{CO}_{2}$ ), one can simply neglect the last term in the numerator. The reactant mixture (ejector inlet) is shown in Figures $5.11 \mathrm{a}$ and $5.12 \mathrm{a}$ while the composition of the post-combustion mixture (mixed-flow plane) is presented in Figures $5.11 \mathrm{~b}$ and $5.12 \mathrm{~b}$. It should be mentioned that nitrogen is not shown in Figures 5.11a and 5.11b since its mass fraction did not change as a result of the secondary combustion. For the present conditions, the $N_{2}$ mass fraction is about 0.3 .

Studying Figures $5.11 \mathrm{~b}$ and $5.12 \mathrm{~b}$ an inflection point is evident in the mass fractions of nearly all of the product species which occurs at an equivalence ratio of 1.2. This point signifies that there is a shift in the chemical reactions which are occurring and divides the graphs into two distinct regions. The first region occurs for $\phi_{r} \leq 1.2$ and will be referred to as oxygen rich since left over $\mathrm{O}_{2}$ shows up in the outflow composition (Fig. 5.11b). The second region spans most of the fuel rich regime $\left(\phi_{r}>1.2\right)$ and will be referred to as oxygen depleted.

In the oxygen rich zone, Fig. 5.11b shows that more of the oxygen becomes depleted as the equivalence ratio increases. From Figure 5.11a one can see that $C O$ appears in the reactant mixture, while the product mixture (Fig. 5.11b) shows no traces of this species for $\phi_{r} \leq 1.2$. Comparing Figures 5.11b and 5.11a it is evident that there is a significant production of $\mathrm{CO}_{2}$ that is occurring in this region. Since 


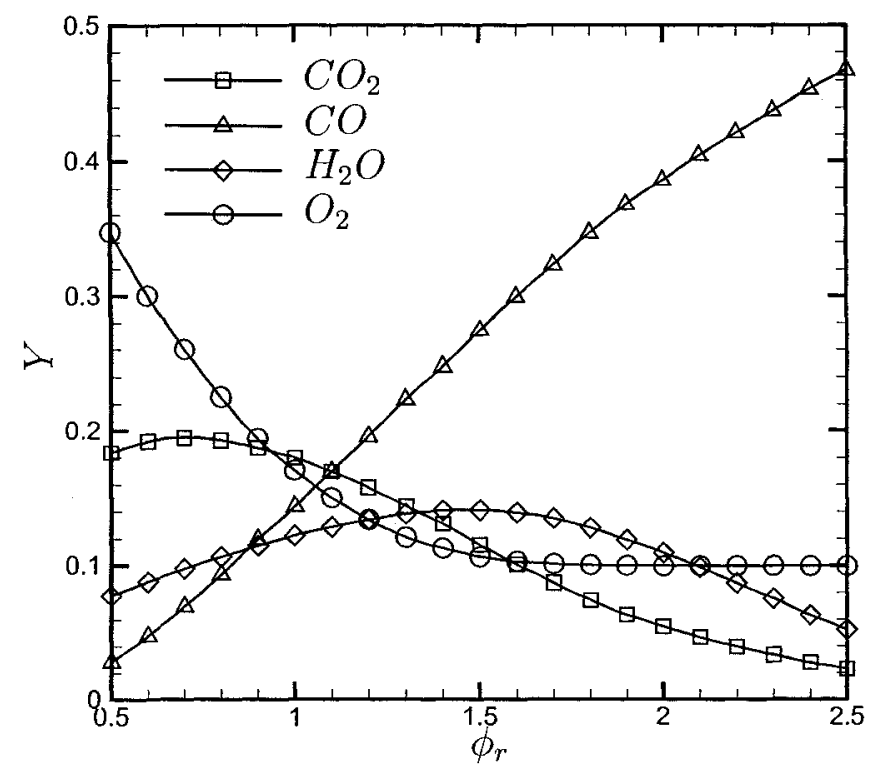

(a) Inlet

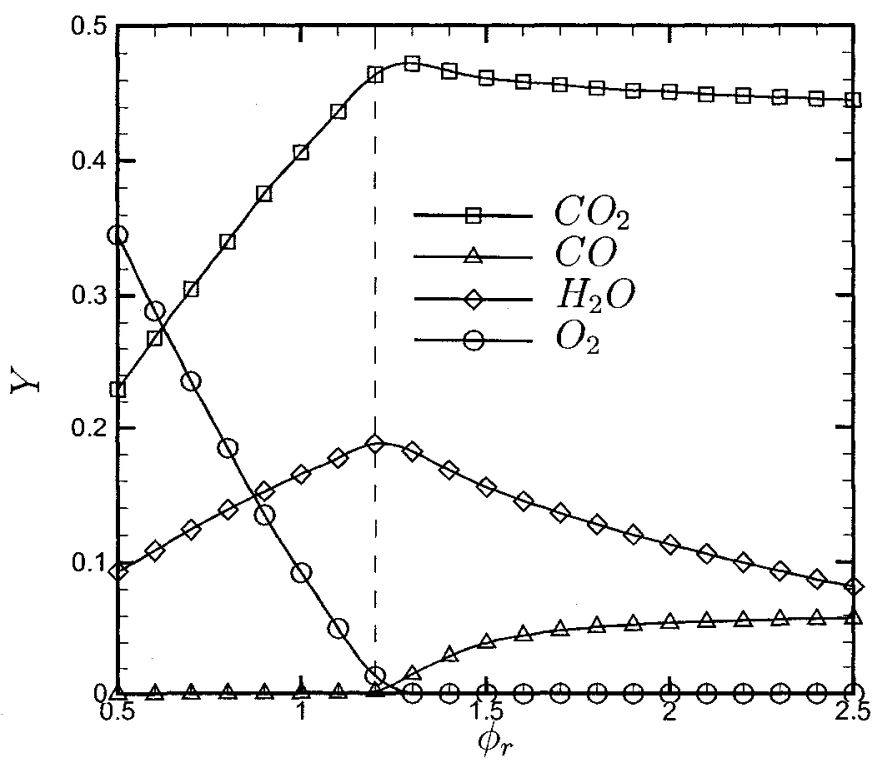

(b) Post-Combustion

Figure 5.11: Ejector Composition - Major Species $\left(C_{R}=0.15\right)$ 
all other carbon-containing species at the ejector inflow occur in negligible amounts, the $\mathrm{CO}$ thus becomes the only source of carbon for the formation of carbon dioxide. In addition to this, Figure 5.12a indicates that there are negligible amounts of $\mathrm{H}_{2}$ entering the ejector in the oxygen rich zone, which means that the incoming oxygen is reacting with the $\mathrm{CO}$ to form $\mathrm{CO}_{2}$.

$$
\mathrm{CO}+\frac{1}{2} \mathrm{O}_{2} \rightarrow \mathrm{CO}_{2} \quad \text { (exothermic) }
$$

Additionally, there is an appreciable amount $\left(Y_{O} \approx 0.014\right)$ of mono-atomic oxygen present in the reactant mixture which is then consumed completely inside the ejector. In light of this, it is reasonable to assume that the oxidation of $C O$ can also occur in the following manner,

$$
\mathrm{CO}+\mathrm{O} \rightarrow \mathrm{CO}_{2} \quad \text { (exothermic) }
$$

There is also a noticeable increase in the mass fraction of $\mathrm{H}_{2} \mathrm{O}$ that is occurring in the oxygen rich zone. However, since Figure 5.12a indicates that there is not enough $\mathrm{H}_{2}$ available for the formation of $\mathrm{H}_{2} \mathrm{O}$ and that the $\mathrm{O}_{2}$ is reacting with carbon monoxide means that the water is being formed through a different route. In the same region, a comparison of Figures 5.12b and 5.12a indicates that there is an order of magnitude reduction in the mass fraction of the hydroxyl radical $(\mathrm{OH})$. Although only a small amount of $H$ is present in the reactant mixture, Fig. 5.12b indicates that this species is being depleted completely. Accordingly, one can infer that the water is being formed by a radical recombination of $O H$ and $H$ which can be written as,

$$
\mathrm{OH}+\mathrm{H}+\mathrm{M} \rightarrow \mathrm{H}_{2} \mathrm{O}+\mathrm{M} \quad \text { (exothermic) }
$$

In the oxygen depleted zone $\left(\phi_{r}>1.2\right)$, the mass fraction of carbon dioxide reaches 


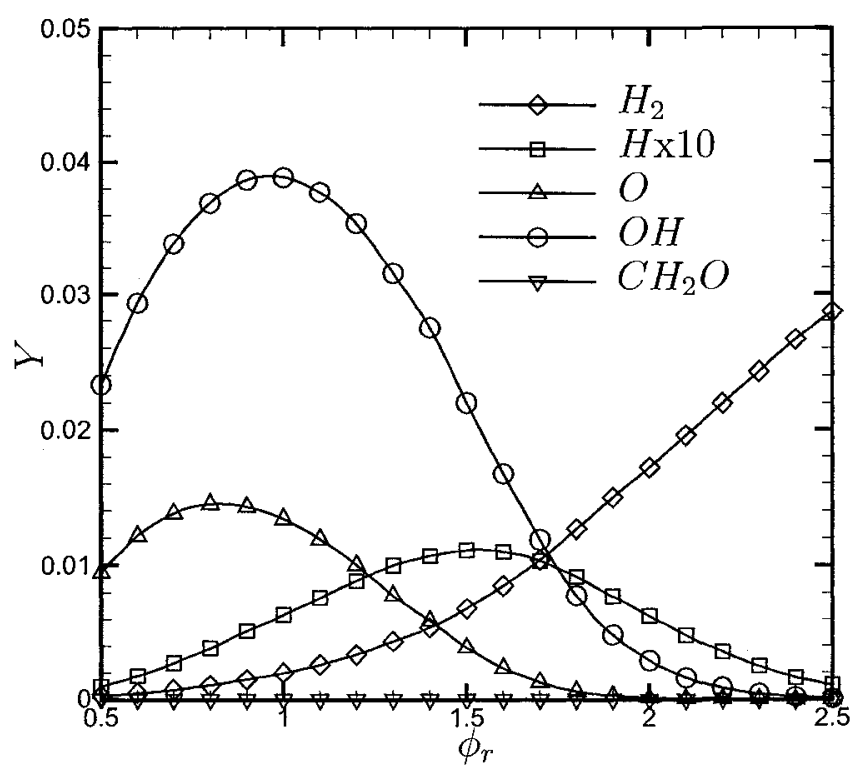

(a) Inlet

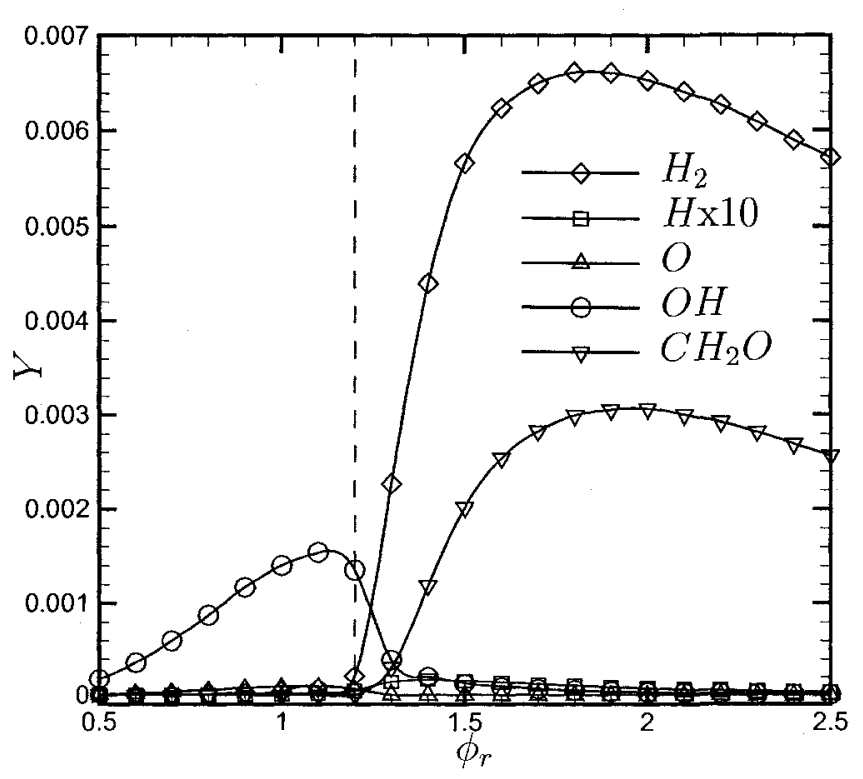

(b) Post-Combustion

Figure 5.12: Ejector Composition - Minor Species $\left(C_{R}=0.15\right)$ 
a value of $Y \approx 0.48$ which stays fixed as the rocket equivalence ratio increases further. On the inlet side, however, the amount of incoming $\mathrm{CO}_{2}$ decreases with $\phi_{r}$ indicating that carbon dioxide production continues throughout the oxygen depleted zone as well. In this region the amount of incoming $C O$ continues to increase while the $\mathrm{O}_{2}$, which now solely comes from the entrained air, stays constant near $Y=0.1$. As a direct consequence, unreacted carbon monoxide can be seen to appear in Figure $5.11 \mathrm{~b}$ since there is no longer enough oxygen to oxidize all of the $C O$. The fraction of $C O$ that appears is quite small $(Y \approx 0.06)$ which means that other reactions may be present which are consuming the excess. Comparing Figures $5.12 \mathrm{~b}$ and $5.12 \mathrm{a}$ it is evident that the $H_{2}$ (mass fraction of $H_{2}$ is approximately halved) which now appears in appreciable amounts in the reactant mixture, is being consumed as well. In the same equivalence ratio range, $\left(\phi_{r}>1.2\right)$, the ejector's product composition begins to show trace amounts of formaldehyde $\left(\mathrm{CH}_{2} \mathrm{O}\right)$, a species which did not appear at all in the inlet mixture (see Fig. 5.12a). The reaction which is occurring in this case is therefore,

$$
\mathrm{CO}+\mathrm{H}_{2} \rightarrow \mathrm{CH}_{2} \mathrm{O} \quad \text { (exothermic) }
$$

One final point which can be made about Figure $5.11 \mathrm{~b}$ is that the mass fraction of water in the oxygen depleted region tends to decrease as $\phi_{r}$ increases. Although the equivalence ratio at which the peak mass fraction of $\mathrm{H}_{2} \mathrm{O}$ occurs differs between the product and reactant mixtures, both show a decrease of $\Delta Y_{\mathrm{H}_{2} \mathrm{O}} \approx-0.09$ between $1.2<\phi_{r}<2.5$. In Figure 5.13 one can in fact see that the curves for the inlet and outlet mass fractions of water begin to converge toward one another in the oxygen depleted zone, and overlap for values of $\phi_{r} \geq 1.6$, diverging only slightly near $\phi_{r}=2.5$. This indicates that the water is not being consumed in a chemical reaction, and that the decrease in the amount of water at the mixed-flow plane is simply caused by a decrease in the amount coming into the ejector. This also means that the $O H / H$ 
recombination is no longer occurring, since the mass fractions of $H$ and $O H$ at the ejector inlet are decreasing with $\phi_{r}$, and no excess water is being formed.

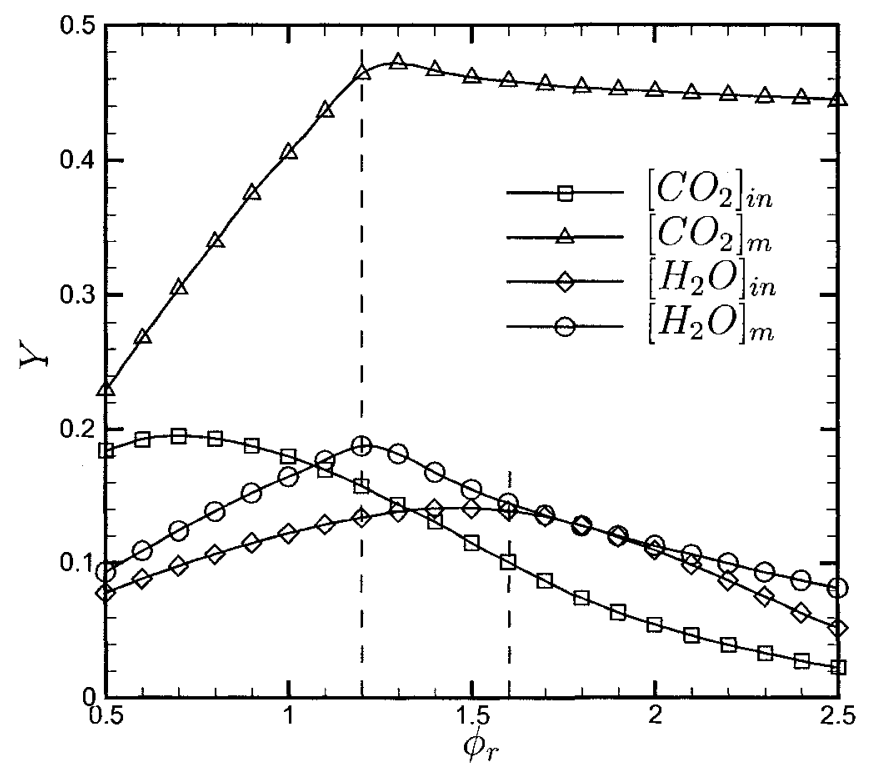

Figure 5.13: Production of $\mathrm{CO}_{2} \& \mathrm{H}_{2} \mathrm{O}\left(C_{R}=0.15\right)$

The chemical reactions that have been mentioned so far are ultimately responsible for the way the combustion process responds to a changing equivalence ratio. It is no accident that the peak combustion temperature (Fig. 5.9) occurs at the same equivalence ratio where an inflection in the product composition was noted. The heat release inside the ejector is caused by the formation of $\mathrm{CO}_{2}$ and $\mathrm{H}_{2} \mathrm{O}$ since they were shown to be the most abundant in the product composition. Although the heats of reaction for the formation of these species are in the same range of values, the oxidation of $C O$ is much more energetic $\left(\phi_{r}=1\right)$, with $\Delta H_{R}=-48,187 \mathrm{~kJ} / \mathrm{kmol}$ as compared to $\Delta H_{R}=-20,202 \mathrm{~kJ} / \mathrm{kmol}$ for the recombination of $H$ and $O H$. The $35 \%$ drop in $\Delta T_{c}$ that occurs when the equivalence ratio exceeds a value of 1.2 can therefore be attributed to the fact that $\mathrm{H}_{2} \mathrm{O}$ is no longer being formed in the oxygen depleted region due to the decreasing amounts of $O H$ and $H$ required for its formation 
as per the reaction previously described. What this means is that $65 \%$ of the heat is due to the oxidation of $C O$ into carbon dioxide, a reaction which is clearly prevalent (see Fig. 5.13) throughout the entire equivalence ratio range considered in the present analysis.

\subsection{Effects of Mixing}

The mixing process inside the ejector is very important since it ultimately determines the amount of air that can be entrained as well as the compressive performance. The fully mixed-theory is adequate for predicting performance under idealized conditions, since a real ejector would be designed to approach those theoretical limits. What the fully mixed approach cannot predict is the duct length required to reach those conditions. Although the CFD results presented earlier can be used as a guide for selecting an appropriate $L / D$, they encompass a limited range of conditions. Consequently an $L / D=5$ may not be sufficiently long for a different ejector configuration. The partially-mixed theory can thus be used to obtain an estimate of the $L / D$ required to achieve complete mixing as well as to quantify the performance loss due to incomplete mixing.

On a physical basis there is a fundamental difference between the governing equations for a fully mixed and a partially mixed ejector. More specifically, one represents an ideal case, which actual ejectors approach, while the partially mixed case is an attempt to include some of the physics of a compressible turbulent mixing layer. Mathematically, however, the only difference between the equation sets are the two mixing parameters, $\beta_{m}$ and $\beta_{e}$. In a sense, it is through these two parameters that the essential physics of mixing enter into the one-dimensional form of the governing equations. For this reason, it is worthwhile to first look at the functional behaviour of the mixing parameters to gain insight into the solution space. Presented below in 
Figures $5.14 \& 5.15$ are the momentum and energy mixing factors respectively.

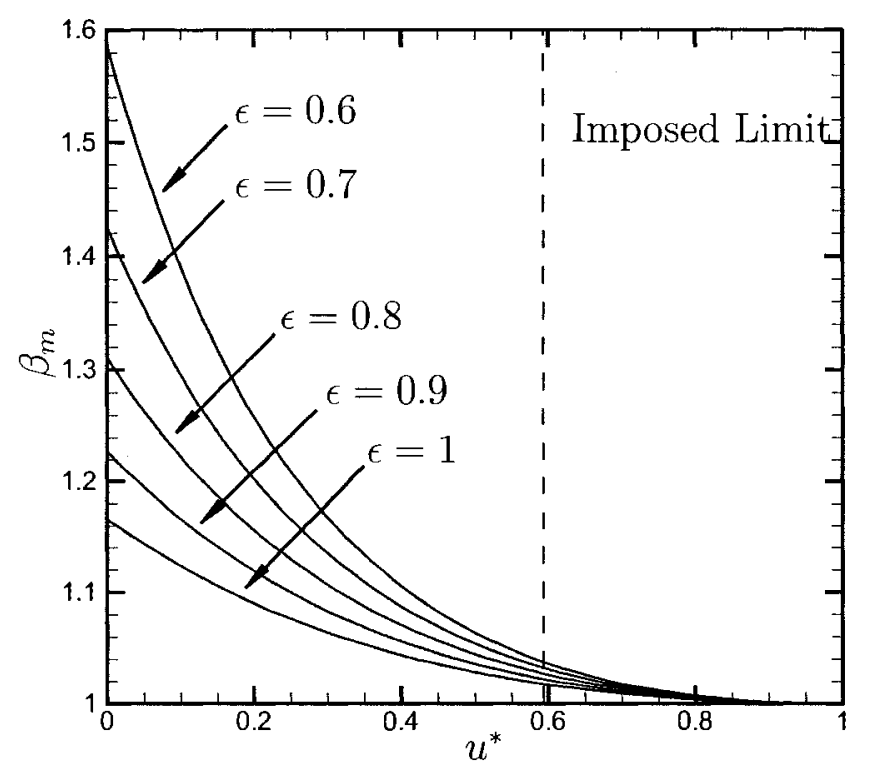

Figure 5.14: Momentum Mixing Parameter

Examining the two figures, it is evident that the curves for both mixing factors have similar shapes, and only differ in the limiting values which implies that either one can be used to judge the extent of mixing in an ejector. One can also see that the width of the shear layer has a strong effect on $\beta_{m}$ and $\beta_{e}$, and tends to flatten out the curves for successively higher values of $\epsilon$. This occurs because increasing the width of the shear layer stretches out the velocity profile in the radial direction which has a strong effect on its curvature. The effect is highly pronounced when $u^{*} \leq 0.6$ and is less significant for higher values of $u^{*}$ when all of the curves collapse onto one another.

Both $\beta_{m}$ and $\beta_{e}$ also display asymptotic behaviour and approach their theoretical limiting values of 1 and 0.5 respectively as $u^{*}$ approaches unity. The limits represent a uniform velocity profile which would be attained if sufficient axial distance was available. How close both mixing factors can approach to their limiting values is 


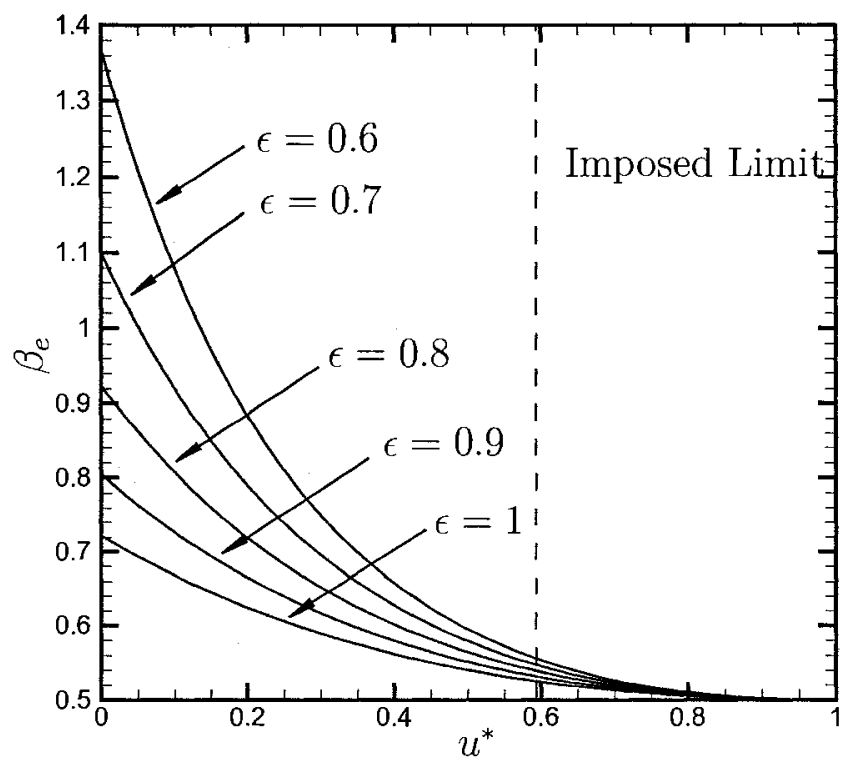

Figure 5.15: Energy Mixing Parameter

governed by the correlation of Alber \& Lees [1]. In the limit, as the axial distance becomes large, Eq. (4.28) approaches a value of $u^{*} \approx 0.593$. For the case when $\epsilon=1$ this would give the imposed limiting values of 1.018 and 0.526 for $\beta_{m}$ and $\beta_{e}$ respectively. This means that regardless of the ejector configuration used, the partially mixed theory cannot actually yield a completely uniform velocity profile. However, since the difference between the theoretical and imposed limiting values of the mixing factors is on the order of $2 \%$, it is expected that the errors associated with calculating various ejector performance parameters such as $\pi_{m}$ or $\alpha$ would be negligibly small and would only occur for configurations with long mixing ducts.

With a good grasp of the mathematical behaviour of the model, one can now proceed to analyze the ejector under conditions of incomplete mixing. The configuration that is examined is the one outlined in Table 5.1 with the constriction ratio fixed at $15 \%$. The compression augmentation factor $\left(\bar{\pi}_{m}\right)$ is plotted as a function of the duct length along with the mixing parameter in Figure 5.16. In this case, the compression 


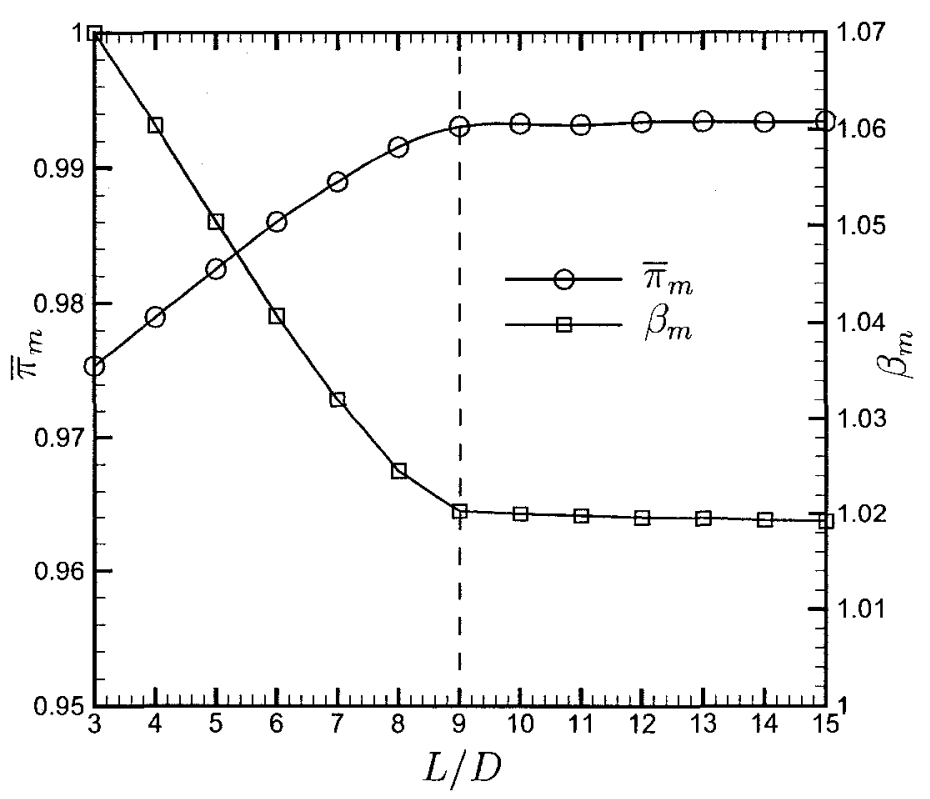

Figure 5.16: Effect of Ejector Duct Length

of an equivalent fully-mixed configuration was used to calculate $\bar{\pi}_{m}$. The reference configuration did not include the effects of secondary combustion since the equivalence ratio was set to $\phi_{r}=0.2$. The data in Figure 5.16 suggests that the extent of mixing varies very little for the $L / D$ range considered. The momentum mixing factor varies by no more than $5 \%$ while $\bar{\pi}_{m}$ stays within an envelope of $2.5 \%$. A difference of this magnitude in the ejector's compression at $L / D=5$ further supports the notion that the discrepancy noted in Figure 5.3 between the theoretical and computational results was not caused by incomplete mixing.

As one might expect from the preceding discussion the compression augmentation ratio never reaches unity, but comes within $0.5 \%$ of the fully mixed case. In light of this discrepancy one must define the mixing length as the point where the extent of mixing stops changing. This gives a mixing length of $L / D=9$, which is in good agreement with published experimental values [19]. It should also be noted that the shape of the $\bar{\pi}_{m}$ curve would differ in reality due to the effects of skin friction, which 
would manifest in two ways. The first, is that the compression ratio would be lower, as was the case with the results of Papamoschou [57] were the inclusion of wall skin friction tended to lower the thrust of the ejector. Secondly, the compression ratio would decrease with $L / D$ once the mixing length is exceeded due to the increase in skin friction with duct length.
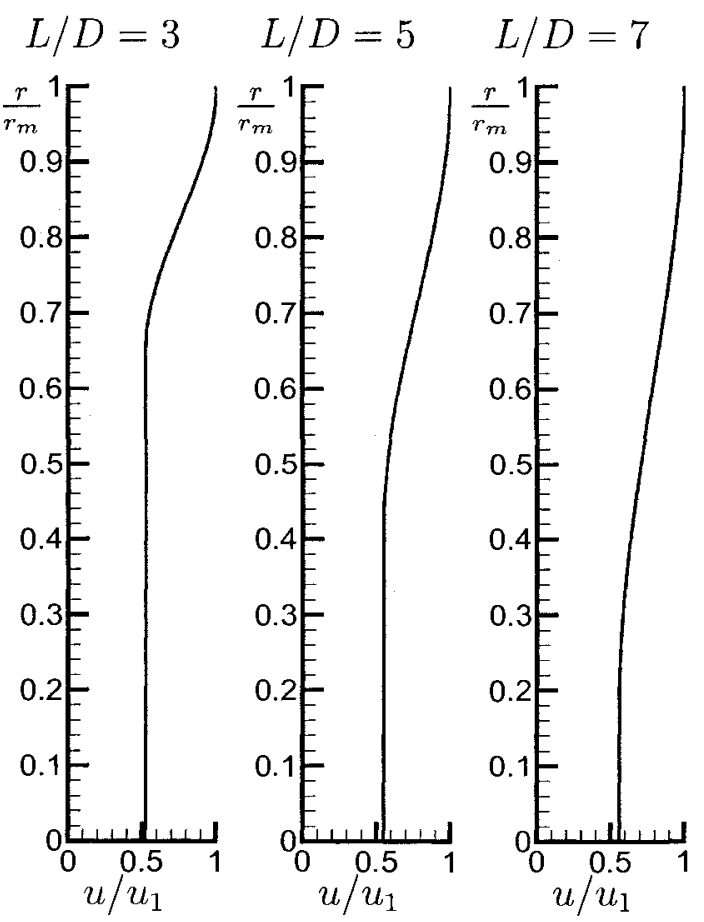

$$
L / D=9
$$$$
L / D=12
$$
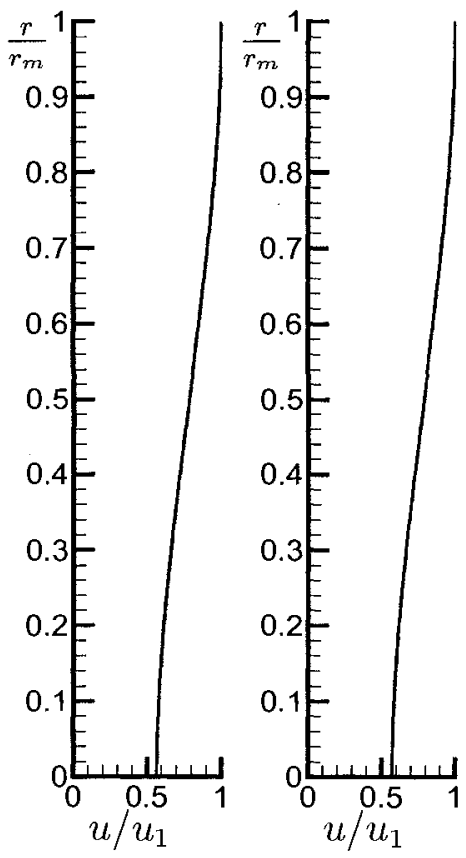

Figure 5.17: Velocity Profiles at Mixed-Flow Plane for Various $L / D$ 's

Velocity profiles at the mixed-flow plane are plotted in Figure 5.17 for various lengths of ejector ducts. The velocity $u_{1}$ represents the velocity at the upper boundary of the shear layer, while the velocity at the lower boundary is simply $u^{*}$. Unlike the data in Fig. 5.16, there is a significant difference in the shape of each of the profiles. For the shortest ducts $(L / D=3,5)$ it is evident that the shear layer has grown to only a small fraction of the duct area and the velocity profiles are dominated by the uniform flow region. For $L / D \geq 9$ the shear layer is significantly thicker and occupies 
the entire mixed-flow plane. It is interesting to note that despite the large variation in $\epsilon$ for all the velocity profiles, the value of $u^{*}$ changes by less than $10 \%$ between the longest and shortest ducts. This is related to Eq. (4.28) which is plotted in Figure 5.18 to produce an s-shaped curve. The cases considered in the figure all fall onto the upper part of the curve, which is why $u^{*}$ changes so little. Thus the main driver for the change in the values of $\beta_{m}$ is the shear layer width, which can be seen to have a strong influence on the curvature of the profiles shown in Figure 5.17.

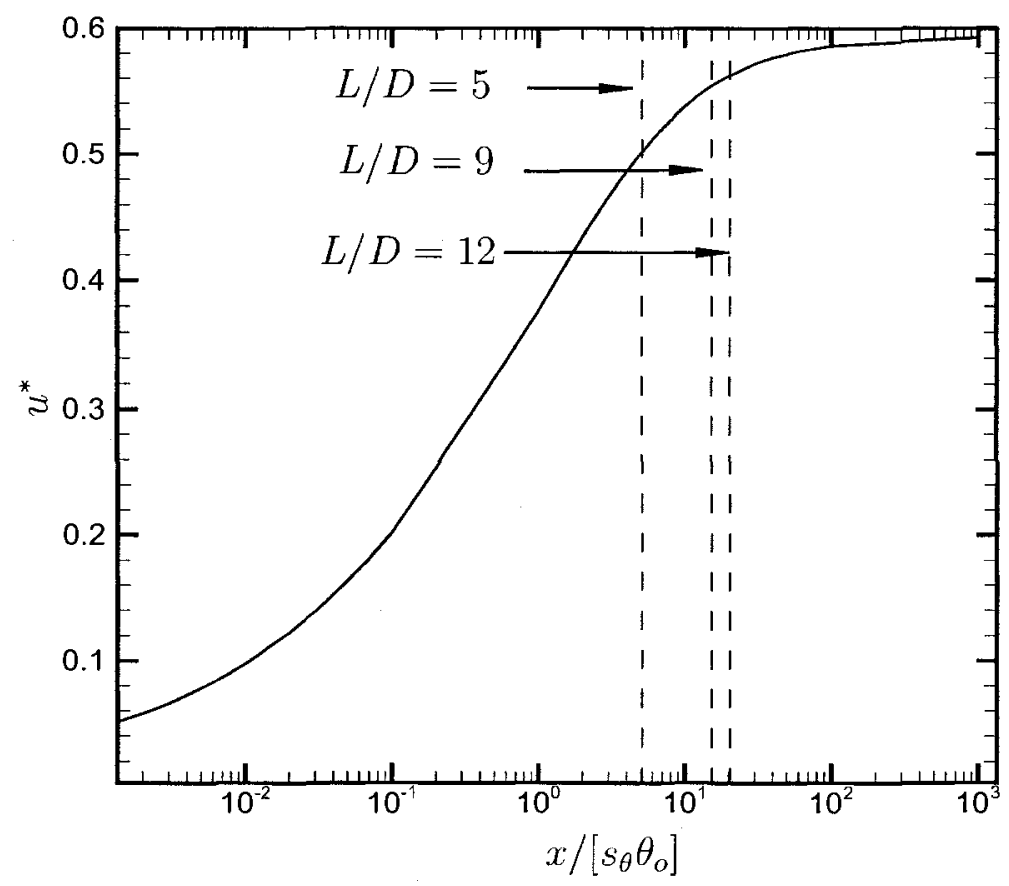

Figure 5.18: Velocity $u^{*}$ from Eq. (4.17)

Whether the mixing length prediction is accurate or not depends on the validity of the assumptions on which the model is built. In the model it is assumed that the density and temperature remain uniform at the outflow plane and that the effects of the wall boundary layer are negligible. The validity of these assumptions depends on the length of the duct being considered. For shorter ducts, the temperature and 
density profiles may no longer be uniform while the effects of the wall boundary layer would become important for longer ducts. Since the temperature and density profiles in the CFD results were uniform for $L / D=5$, it is therefore reasonable to assume that those effects would not alter the prediction of the model. The wall boundary layer, on the other hand, may be fairly thick for $L / D=9$ and although it would increase the mixing factor, the extent of the mixing would remain unchanged. In addition to these considerations, one must also keep in mind that the model uses a correlation for a planar shear layer to estimate the mixing extent in an axisymmetric case. Since axisymmetric shear layers tend to spread out faster [64], the mixing length predicted by the model is most likely a conservative estimate.

\subsection{Thrust Augmentation}

The thrust of an ejector depends not only on how effectively it compresses the incoming air but also on how much air it entrains. The results presented so far have only dealt with the compression ratio, since $\alpha$ was held fixed. To quantify the effects of $C_{R}$ and $\phi_{r}$ in a more complete manner, one needs to look at the how well the ejector augments thrust. The analysis will therefore consider a fully-mixed ejector under SMC conditions while using the case when combustion does not occur $\left(\phi_{r}=0.2\right)$ as a reference. The ejector configuration is modified to include a rocket that is representative of an Atlas E/F first stage (Rocketdyne MA-3 engine), with the pertinent parameters shown in Table 5.4.

The ejector configuration used for thrust calculations is shown in Figure 5.19 and is fitted with a diverging propelling nozzle since the mixed-flow Mach number is fixed at unity for all cases. The flow entering the nozzle at plane $m$ is assumed to expand isentropically to atmospheric pressure $\left(p_{\infty}\right)$ by the time it reaches plane $e$. Given that the pressures at either end of the nozzle are specified $\left(p_{m}, p_{e}\right)$, and $p_{m}$ can vary 


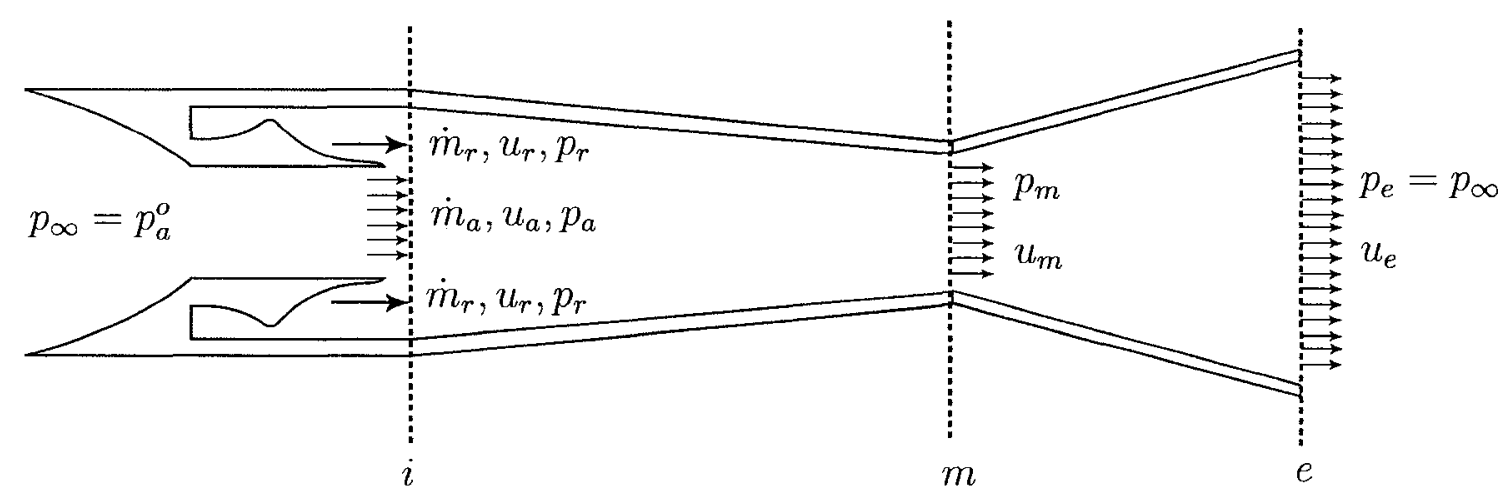

Figure 5.19: Calculation of Ejector Thrust

implies that the area ratio of the nozzle can vary as well. The thrust of the ejector is then calculated by taking the difference in the momentum fluxes and pressure forces between planes $i$ and $e$. The thrust of the rocket is calculated by considering the rocket in isolation and subtracting the air ram $\operatorname{drag}\left(p_{\infty} A_{r}\right)$ from the pressure force and momentum flux at the rocket's exit plane. The thrust augmentation ratio $\Phi$, is calculated by dividing the thrust force of the ejector by that of the rocket.

\section{Table 5.4: Ejector Configuration}

\begin{tabular}{lc|lc}
\hline \hline$M_{m}$ & 1 & $T_{a}^{o}[\mathrm{~K}]$ & 279 \\
$M_{r}$ & 3 & $\sigma$ & 0.1 \\
$p_{r}^{o}[\mathrm{~atm}]$ & 48 & $r_{i}[\mathrm{~m}]$ & 1 \\
$p_{a}^{o}[\mathrm{kPa}]$ & 100 & $L / D$ & 5 \\
\hline \hline
\end{tabular}

The fully mixed configuration is depicted in Figure 5.20 at static sea-level conditions. Examining Figure 5.20, one notes that the shapes of the curves display an upper limit in constriction past which thrust augmentation begins to decrease. The limit occurs roughly near $C_{R} \approx 0.55$, and varies by no more than $10 \%$ for different equivalence ratios, with the Stoichiometric and reference cases $\left(\phi_{r}=0.2\right)$ showing the widest operating ranges. This maximum represents the theoretical performance 
limit for the present ejector configuration, and occurs because area constriction, while improving compression, tends to limit the maximum amount of air that can be entrained.

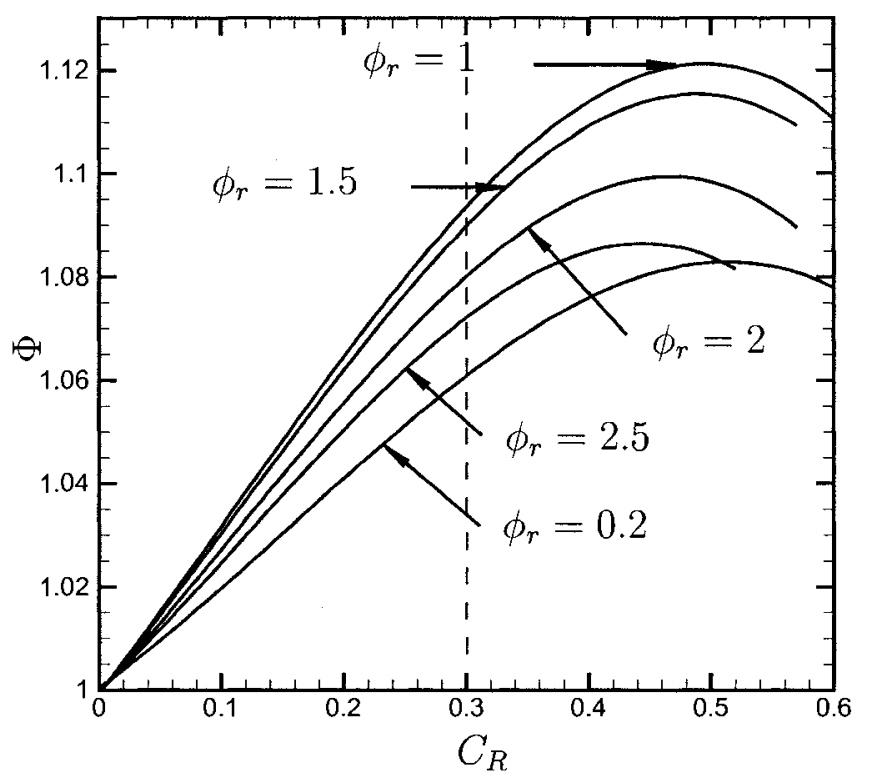

Figure 5.20: Ejector Performance Map

Figure 5.20 also shows that the rocket equivalence ratio has an effect on the thrust augmentation of the ejector. To present this trend more clearly, $\Phi$ is plotted as a function of $\phi_{r}$ in Figure 5.21, which shows that thrust augmentation is maximized at Stoichiometric conditions. As more fuel is added, thrust augmentation drops fairly rapidly, with the $\phi_{r}=2.5$ curve showing nearly a $4 \%$ drop from the Stoichiometric case. The reason that the best performance occurs at Stoichiometric conditions and not at $\phi_{r}=2.5$, as was the case with $\pi_{m}$ in Fig. 5.5 is tied to the entrainment ratio, which is no longer fixed in this case. Since sonic conditions are maintained at the mixed-flow plane for all configurations, the ejector is essentially operating at maximum mass flow conditions at each value of $C_{R}$. This means that in addition to the compression factor, the combustion inside the ejector is free to adjust the 
entrainment ratio as well.

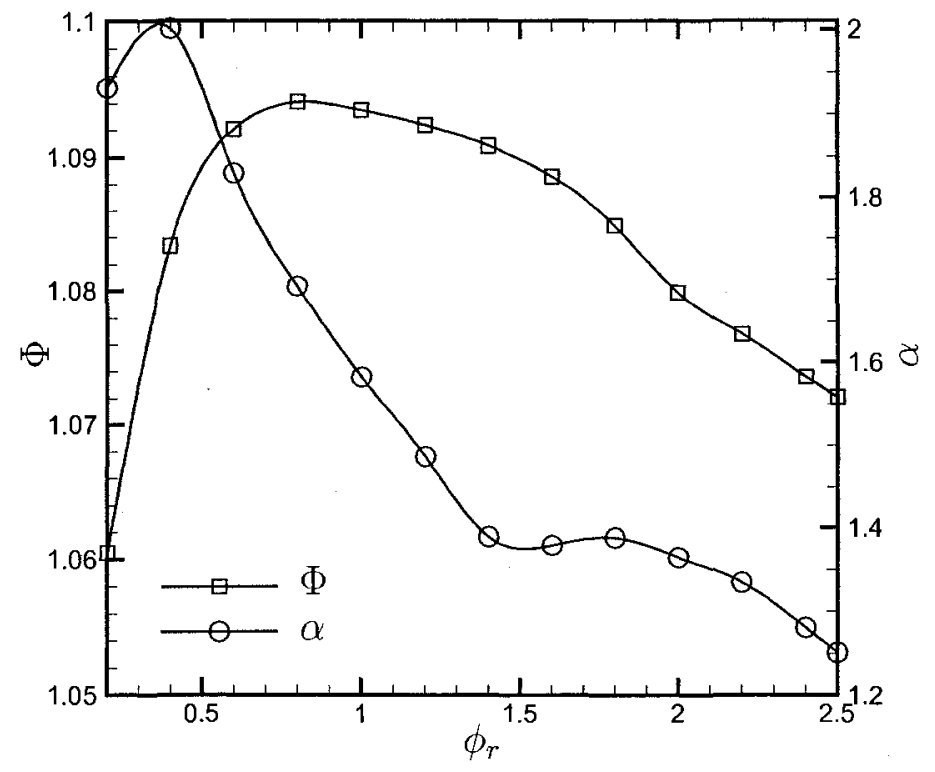

Figure 5.21: Thrust Augmentation and Air Entrainment $\left(C_{R}=0.3\right)$

Figure 5.21 displays the entrainment ratio for the same conditions shown in Table 5.4. From the figure, it is clear that for the given conditions the combustion inside the ejector is limiting the amount of air that is being entrained, with higher values of $\phi_{r}$ yielding progressively lower values of $\alpha$. Comparing the two curves in Fig. 5.21 it becomes clear that the rocket equivalence ratio affects $\alpha$ differently than $\Phi$, which means that it is not $\alpha$ alone that is causing the trends shown in Fig. 5.20. To explore this idea further one needs to consider the thrust augmentation ratio itself, which can be approximated as,

$$
\Phi=\frac{(\alpha+1) u_{e}-\alpha u_{a}}{u_{r}}
$$

if one neglects the effects of pressure forces. From the equation, it is clear that in addition to $\alpha$, the thrust augmentation is strongly dependent on the velocity at the exit plane of the propelling nozzle, $u_{e}$. The fact that the expansion in the nozzle is 
isentropic implies that $u_{e}$ is proportional to the mixed-flow velocity, $u_{m}$. Referring back to Figure 5.8 one notes that the maximum mixed-flow Mach number occurred near Stoichiometric conditions ( $\phi_{r}=1.2$ to be precise). Combining this with the fact that the maximum temperature occurred at the same value of $\phi_{r}$ yields a mixed-flow velocity that is nearly double that of the reference case. On the other hand, Figure 5.21 shows that $26 \%$ more air is entrained at reference conditions as compared to the Stoichiometric case. The net effect of the two is a higher thrust augmentation ratio, since the nearly two-fold increase in $u_{m}$ offsets the $26 \%$ drop in $\alpha$. At higher equivalence ratios, both the mixed-flow velocity and the entrainment ratio are diminished, thus causing the lower levels of thrust present at those conditions. 


\section{Chapter 6}

\section{Conclusions}

A theoretical treatment for a constricted ejector has been presented. The bulk of the theory relied on the assumption of complete mixing and used an equilibrium calculation procedure to analyze the SMC operating regime. For a non-reacting case, modifications to the preceding theory were introduced to account for incomplete mixing and its effects on overall performance. In either case, a wall pressure distribution needed to be assumed to close the system of equations. Several options for this pressure distribution were presented, namely two analytical functions (linear \& polynomial) as well as wall pressures extracted directly from CFD simulations.

Other than the inlet and exit pressures, the polynomial pressure distribution required that the minimum rocket pressure, $p_{\min }$ be specified. Two methods for calculating this quantity were presented, one which made use of a Riemann solver and another which made use of a control volume (CV method) formulation. Both techniques predicted very similar values of $p_{\min }$ because of the common assumptions (isentropic flow, no mixing) on which the methods were based. In addition, the analytical solutions for $p_{\min }$ were 60-70\% larger than the CFD prediction due to non-isentropic effects as well as the highly two-dimensional flow in the computational case.

To evaluate which pressure distribution was the most appropriate in terms of 
accuracy, solutions under non-reacting, fully mixed conditions were obtained for the ejector's compression $\left(\pi_{m}\right)$ and compression augmentation $\left(\bar{\pi}_{m}\right)$ factors. Overall, the trends for ejector compression that were obtained were very close to the CFD results, although the analytical values of $\pi_{m}$ were about $30-40 \%$ higher, with turbulent and viscous effects being identified as the major causes. Within the theoretical solutions, it was found that the polynomial pressure distribution was the best approximation for quantifying $F_{p, x}$ since the results it gave for $\pi_{m}$ and $\bar{\pi}_{m}$ were very close to those obtained when the CFD wall pressures were used.

Further analysis of the fully mixed ejector was performed under SMC conditions by varying the equivalence ratio of the rocket in the range $0.2<\phi_{r}<2.5$. Operation in this mode caused significant thermal choking for $\phi_{r} \geq 1$ which reduced the allowable range of $C_{R}$ by $14 \%$ in the worst case, and was directly related to the combustion temperature rise inside the ejector. The combustion also affected the ejector's compression ratio in a manner which depended on the equivalence ratio at which the rocket was operating. At Stoichiometric conditions, there was an $8 \%$ drop in $\pi_{m}$ which was caused by a lower mixed-flow pressure, while the highest compression ratio occurred at an equivalence ratio of 2.5 due to an increased Mach number. For all values of $\phi_{r}>0.5$ the effects of the secondary combustion were significant and were responsible for about $40 \%$ of the changes noted in the ejector's mixed-flow temperature and compression ratio.

Examination of the ejector's post-combustion mixture revealed the existence of an inflection point in the mass fractions of nearly all of the product species which occurred at $\phi_{r}=1.2$ and effectively split the equivalence ratio range into two zones. The oxidation of $\mathrm{CO}$ and the recombination of $H$ and $O H$ where shown to be the reactions primarily responsible for the heat release inside the ejector. While the Carbon Monoxide reaction persisted throughout the entire equivalence ratio range, the radical recombination was mainly active in the oxygen rich zone, and is in fact 
what caused the inflection point and the subsequent drop in the heat release at higher equivalence ratios.

The partially mixed theory was used to estimate the mixing length required to achieve fully mixed conditions as well as to quantify the drop in compression caused by incomplete mixing. For long mixing ducts, it was shown that the model was not able to produce a completely uniform velocity profile due to the correlation used to define $u^{*}$. Although a mixing duct with an $L / D=5$ yielded a flow that was $95 \%$ mixed with a compression ratio that was only $2.5 \%$ lower than the fully mixed value, a limit in the mixing extent was not reached until an $L / D=9$. Furthermore, for the $L / D$ range considered, the drop in $\pi_{m}$ due to incomplete mixing never exceeded $2.5 \%$, further proving that incomplete mixing was not the cause of the discrepancy between the theoretical and the computational results.

The thrust augmenting performance of the ejector was investigated under fully mixed conditions. The case when secondary combustion was absent at $\phi_{r}=0.2$ was used as a reference throughout. The results showed an upper limit in thrust augmentation near $C_{R} \approx 0.55$ past which $\Phi$ decreased. Maximum thrust augmentation was achieved at $\phi_{r}=1$, while operation in the fuel rich regime caused a $4 \%$ drop in $\Phi$ at an equivalence ratio of 2.5. This occurred because compared to the reference case, there was a two-fold increase in the mixed-flow velocity at Stoichiometric conditions while $\alpha$ decreased by only $26 \%$. 


\subsection{Future Work}

In light of the results reported in this study there are several routes which any research that follows can take. The fully-mixed theory that was outlined in this work is complete and should not be developed further. The best course of action would be to use the theory to obtain performance data under operating conditions not covered in the present work. An example of this would be to look at the DAB mode of operation as well as to find the optimum operating parameters for this mode. Alternatively one can use the fully-mixed theory in an optimization routine of some sort to generate one or more optimized ejector configurations.

The partially-mixed theory, on the other hand, should be extended to include the effects of non-uniform temperature and density profiles for instance. Although not crucial, a correlation for $u^{*}$ which is better suited to the present application should be found to ensure that a fully mixed case can be reached under the right operating conditions. One can also modify the theory slightly to account for the effect of wall skin friction, which would provide a more realistic prediction of the ejector's performance.

The Riemann approach that was introduced also warrants further investigation, to see if it alone can be used to predict the ejector's performance. Since the Riemann approach essentially solves the ejector flowfield in a piece-wise manner, it is therefore well suited for use in a more complex, differential ejector model, similar to the one suggested by Papamoschou [57]. The advantage of using the Riemann solver in this manner would be that one would no longer have to consider an ejector where the pressures of the two streams are matched. 


\section{Bibliography}

[1] Alber, I. E. and Lees, L., "Integral Theory for Supersonic Turbulent Base Flows," AIAA Journal, Vol. 6, No. 7, 1968.

[2] Alperin, M. and Wu, J. J., "Thrust Augmenting Ejectors, Part I," AIAA Journal, Vol. 21, No. 10, 1983.

[3] Anderson, J. D., Fundamentals of Aerodynamics, McGraw Hill, 3rd ed., 2001.

[4] Anderson, J. D., Modern Compressible Flow, McGraw Hill, 3rd ed., 2002.

[5] Anderson, J. D., Computational Fluid Dynamics, McGraw Hill, 1995.

[6] Aoki, S., Lee, J., and Masuya, G., "Aerodynamic Experiment on an Ejector-Jet," Journal of Propulsion and Power, Vol. 21, No. 3, 2005.

[7] Bartosiewicz, Y., Aidoun, Z., Desevaux, P., and Mercadier, Y., "Numerical and Experimental Investigations on Supersonic Ejectors," Int. Journal of Heat and Fluid Flow, Vol. 26, 2006, pp. 56-70.

[8] Bevilaqua, P. M., "Evaluation of Hypermixing for Thrust Augmenting Ejectors," Journal of Aircraft, Vol. 11, No. 6, 1974.

[9] Bond, R. B. and Edwards, J. R., "CFD Analysis of the Low Speed Propulsion Mode in an RBCC Engine," Paper 2004-857, AIAA, 2004. 
[10] Bradford, J. E. and Olds, J. R., "SCCREAM v.5: A Web-Based Airbreathing Propulsion Analysis Tool," Paper 99-2104, AIAA, 1999.

[11] Campbell, B. T., Siebenhaar, A., and Nguyen, T., "Strutjet Engine Performance," Journal of Propulsion and Power, Vol. 17, No. 6, 2001.

[12] Chow, W. L. and Addy, A. L., "Interaction Between Primary and Secondary Streams of Supersonic Ejector Systems and Their Performance Characteristics," AIAA Journal, Vol. 2, No. 4, 1964.

[13] Chase, M. W., Davies, C. A., Downey, J. R., Frurip, D. J., Macdonald, R. A., and Syverud, A. N., JANAF Thermochemical Tables, Dow Chemical Company, 3rd ed.

[14] Cramer, J. M., Greene, M., Pal, S., and Santoro, R. J., "RBCC Ejector Mode Operating Characteristics for Single and Twin Thruster Configurations," Paper 2001-3464, AIAA, 2001.

[15] Daines, R. L. and Bulman, M., "Computational Analyses of Dynamic Rocket Ejector Flowfields," Paper 96-2686, AIAA, 1996.

[16] Daines, R. L. and Russel, R. M., "Numerical Analysis of the Effects of Combustion in Rocket Ejectors," Paper 1998-3772, AIAA, 1998.

[17] Daines, R. and Segal, C., "Combined Rocket and Airbreathing Propulsion Systems for Space-Launch Applications," Journal of Propulsion and Power, Vol. 14, No. 5, 1998.

[18] Desevaux, P. and Lanzetta, F., "Computational Fluid Dynamic Modeling of Pseudoshock Inside a Zero-Secondary Flow Ejector," AIAA Journal, Vol. 42, No. 7, 2004. 
[19] Dijkstra, F., Marée, A. G. M., Caporicci, M., and Immich, H., "Experimental Investigation of the Thrust Enhancement Potential of Ejector Rockets," Paper 97-2756, AIAA, 1997.

[20] Dobrowolski, A., "Analysis of Nonconstant Area Combustion and Mixing in Ramjet and Rocket-Ramjet Hybrid Engines," Tn d-3626, NASA, 1966.

[21] duP. Donaldson, C. and Gray, K. E., "Theoretical and Experimental Investigation of the Compressible Free Mixing of Two Dissimilar Gases," AIAA Journal, Vol. 4, No. 11, 1966.

[22] Dutton, J. C. and Carrol, B. F., "Optimal Supersonic Ejector Designs," Journal of Fluids Engineering, Vol. 108, No. 4, 1986.

[23] Dutton, J. C. and Carrol, B. F., "Limitation of Ejector Performance Due to Exit Choking," Journal of Fluids Engineering, Vol. 110, 1988, pp. 91-93.

[24] Dutton, J. C., Mikkelsen, C. D., and Addy, A. L., "A Theoretical and Experimental Investigation of the Constant Area, Supersonic-Supersonic Ejector," AIAA Journal, Vol. 20, No. 10, 1982.

[25] Escher, W. J. D., "The Seven Operating Modes of the Supercharged Ejector Scramjet (SESJ) Combined-Cycle Engine," Paper 2001-3240, AIAA, 2001.

[26] Etele, J., Computational Study of Variable Area Ejector Rocket Flowfields, Ph.D. thesis, University of Toronto, Toronto, 2004.

[27] Etele, J., Parent, B., and Sislian, J. P., "Analysis of Increased Compression Through Area Constriction on Ejector-Rocket Performance," Journal of Spacecraft and Rockets, Vol. 44, No. 2, 2007.

[28] Etele, J. and Sislian, J. P., "Analysis of Increased Compression Factor on EjectorRocket Performance," Paper 2006-5.2.2, ICAS, 2006. 
[29] Etele, J., Sislian, J. P., and Parent, B., "Effect of Rocket Exhaust Configurations on Ejector Performance in RBCC Engines," Journal of Propulsion and Power, Vol. 21, No. 4, 2005.

[30] Fabri, J. and Paulon, J., "Theory and Experiments on Supersonic Air-Air Ejectors," Tm-1410, NACA, 1956.

[31] Fancher, R. B., "Low-Area Ratio, Thrust-Augmenting Ejectors," Journal of Aircraft, Vol. 9, No. 3, 1972.

[32] Foelsche, R. O., Tsai, C. Y., and Bakos, R. J., "Experiments on a RBCC Ejector Scramjet with Integrated, Staged Secondary Fuel Injection," Paper 99-2242, AIAA, 1999.

[33] Fox, R. W. and McDonald, A. T., Introduction to Fluid Mechanics, John Wiley \& Sons, 5th ed., 1998.

[34] Goebel, S. G. and Dutton, J. C., "Experimental Study of Compressible Turbulent Mixing Layers," AIAA Journal, Vol. 29, No. 4, 1991.

[35] Gottlieb, J. J. and Groth, C. P. T., "Assessment of Riemann Solvers for Unsteady One-Dimensional Inviscid Flows of Perfect Gases," Journal of Computational Physics, Vol. 78, 1988, pp. 437-458.

[36] Han, S., Peddieson, J. J., and Gregory, D., "Ejector Primary Flow Molecular Weight Effects in an Ejector Ram-Rocket Engines," Journal of Propulsion and Power, Vol. 18, No. 3, 2002.

[37] Han, S. and Peddieson, J. J., "One-Dimensional Numerical Study of Compressible Flow Ejector," AIAA Journal, Vol. 40, No. 7, 2002, Technical Note.

[38] Han, S., Tomes, J., and Lane, J., "Numerical Study of PSU-RBCC Ejector Mode Operation," Paper 2003-5230, AIAA, 2003. 
[39] Huang, W. and Xing, Y., "A Conceptual Study of RBCC for Hypersonic Missile," Paper 2005-4285, AIAA, 2005.

[40] Jahingir, M. N. and Huque, Z., "Design Optimization of Rocket-Based Combined-Cycle Inlet/Ejector System," Journal of Propulsion and Power, Vol. 21, No. 4, 2005.

[41] Jos, C. C., Anderson, W. E., Sankaran, V., and Gujarathi, A., "Ducted Rocket Tests with a Fuel Rich Primary Thruster," Paper 2005-4282, AIAA, 2005.

[42] Kamhawi, H., Krivanek, T., and Thomas, S. R., "Direct-Connect Ejector Ramjet Combustor Experiment," Paper 2003-16, AIAA, 2003.

[43] Kawatsu, K., Koike, S., Kumasaka, T., Masuya, G., and Takita, K., "PseudoShock Wave Produced by Backpressure in Straight and Diverging Rectangular Ducts," Paper 2005-3285, AIAA, 2005.

[44] Kitamura, E., Mitani, T., Huang, S., and Masuya, G., "Pressure Recovery in Mixing Ducts of Ejector-Ramjets," Paper 2005-3420, AIAA, 2005.

[45] Kitamura, E., Tomioka, S., Sakuranaka, N., and Masuya, G., "Pressure Recovery Characteristics of Diverging Mixing Tubes for Ejector-Jets," Paper 2006-7979, AIAA, 2006.

[46] Lehman, M., Pal, S., Broda, J. C., and Santoro, R. J., "Raman Spectroscopy Based Study of RBCC Ejector Mode Performance," Paper 99-0090, AIAA, 1999.

[47] Lehman, M., Pal, S., and Santoro, R. J., "Experimental Investigation of the RBCC Rocket-Ejector Mode," Paper 2000-3725, AIAA, 2000.

[48] Li, Y. F., He, G. Q., and Liu, P. J., "Experimental Investigation of Rocket Ejector in SMC Combustion Mode," Paper 2006-5042, AIAA, 2006. 
[49] Lineberry, D. and Landrum, B., "Effects of Multiple Nozzles on Asymmetric Ejector Performance," Paper 2005-4283, AIAA, 2005.

[50] Luke, E. A. and Tang, L., "A CFD Benchmark Study for RBCC Ejector Mode Operation," Paper 2005-4425, AIAA, 2005.

[51] Masuya, G., Chinzei, N., and Ishii, S., "A Study of Airbreathing Rockets Subsonic Mode Combustion," Acta Astronautica, Vol. 8, No. 5-6, 1981.

[52] Matsuo, K., Miyazato, Y., and Kim, H. D., "Shock Train and Pseudo-Shock Phenomena in Internal Gas Flows," Progress in Aerospace Sciences, Vol. 25, 1999, pp. 33-100.

[53] McBride, B. J., Gordon, S., and Reno, M. A., "Coefficients for Calculating Thermodynamic and Transport Properties of Individual Species," Tm 4513, NASA, 1993.

[54] O'Brien, T. F. and Lewis, M. J., "Rocket-Based Combined-Cycle Engine Integration on an Osculating Cone Wvaerider Vehicle," Journal of Aircraft, Vol. 38, No. 6, 2001.

[55] O'Brien, T. F., Starkey, R. P., and Lewis, M. J., "Quasi-One-Dimensional HighSpeed Engine Model with Finite Rate Chemistry," Jornal of Propulsion and Power, Vol. 17, No. 6, 2001.

[56] Olds, J. R. and Bradford, J. E., "SCCREAM: A Conceptual Rocket-Based Combined-Cycle Engine Performance Analysis Tool," Journal of Propulsion and Power, Vol. 17, No. 2, 2001.

[57] Papamoschou, D., "Analysis of a Partially Mixed Supersonic Ejector," Journal of Propulsion and Power, Vol. 12, No. 4, 1996. 
[58] Peters, C. E., Phares, W. J., and Cunningham, T. H. M., "Theoretical and Experimental Studies of Ducted Mixing and Burning of Coaxial Streams," Journal of Spacecraft, Vol. 6, No. 12, 1969.

[59] Presz, W. M. J., Morin, B. L., and Gousy, R. G., "Forced Mixer Lobes in Ejector Designs," Journal of Propulsion and Power, Vol. 4, No. 4, 1988.

[60] Quinn, B., "Compact Ejector Thrust Augmentation," Journal of Aircraft, Vol. 10, No. 8, 1973.

[61] Quinn, B., "Ejector Performance at High Temperatures and Pressures," Journal of Aircraft, Vol. 13, No. 12, 1976.

[62] Rojas-Oviedo, R. and Deng, Z. T., "Analysis of Proposed Fully Internal Compression Geometry for a Rocket-Based Combined Cycle Engine," Paper 20010824, AIAA, 2001.

[63] Russel, R. M., Brocco, D. S., and Daines, R. L., "Modeling and Validation of an Ejector Primary Rocket for Shielded Afterburning Fuel Injection," Paper 992241, AIAA, 1999.

[64] Schetz, J. A., Boundary Layer Analysis, Prentice Hall, 1993.

[65] Steffen, C. J. J. and Yungster, S., "Computational Analysis of the Combustion Processes in an Axisymmetric, RBCC Flowpath," Tm 2001-210679, AIAA, 2001.

[66] Tillman, T. G., Paterson, R. W., and Presz, W. M., "Supersonic Nozzle Mixer Ejector," Journal of Propulsion and Power, Vol. 8, No. 2, 1992.

[67] Tokudome, S. and Kobayashi, K., "Experimental Study on Rocket-Ejector Systems," Paper 2006-7977, AIAA, 2006.

[68] Turns, S., An Introduction to Combustion, McGraw Hill, 2nd ed., 2000. 
[69] West, J. and Ruf, J., "Computational Insight into Experimentally Observed Change in Mixing Characteristics of an RBCC Engine in Ejector Mode," Paper 2001-3459, AIAA, 2001.

[70] Yungster, S. and Trefny, C. J., "Analysis of a New Rocket-Based Combined-Cycle Engine Concept at Low Speed," Paper 99-2393, AIAA, 1999.

[71] Zucrow, J. M. and Hoffman, J. D., Gas Dynamics, Vol. 2, John Wiley \& Sons, 1977. 